\title{
Fluctuation effects on 3D Lagrangian mean and Eulerian mean fluid motion
}

\author{
Darryl D. Holm \\ Theoretical Division and Center for Nonlinear Studies \\ Los Alamos National Laboratory, MS B284 \\ Los Alamos, NM 87545 \\ email: dholm@lanl.gov \\ To appear, Physica D
}

\begin{abstract}
We formulate equations for the slow time dynamics of fluid motion that self consistently account for the effects of the variability upon the mean. The time-average effects of the fluctuations introduce nonlinear dispersion that acts to spatially smooth the transport velocity of the mean flow relative to its circulation or momentum velocity, by the inversion of a Helmholtz operator whose length scale corresponds to the covariance of the fluctuations.
\end{abstract}




\section{Contents}

1 Introduction 3

1.1 Lagrangian mean equations . . . . . . . . . . . . . . . . . . 4

1.2 Eulerian mean equations . . . . . . . . . . . . . . . . . . . . 8

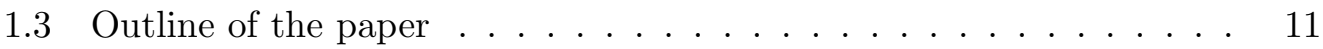

2 Review of Hamilton's principle and Euler-Poincaré equations for ideal fluids

\begin{tabular}{|lll}
3 & Averaged Lagrangians and Euler-Lagrange equations & 14
\end{tabular}

3.1 Lagrangian fluid trajectory fluctuations . . . . . . . . . . . . . . . . 14

3.2 Induced Eulerian velocity fluctuations . . . . . . . . . . . . . . . 15

3.3 A Taylor series approximation to order $O\left(|\boldsymbol{\xi}|^{2}\right)$. . . . . . . . . . . 16

$3.4 \quad$ Remarks about advected quantities and Taylor's hypothesis . . . . . 18

3.5 Averaged approximate Lagrangiand . . . . . . . . . . . . . . . . . 19

3.6 Further approximations . . . . . . . . . . . . . . . . 20

3.7 The Euler-Lagrange equations with order $O\left(|\boldsymbol{\xi}|^{2}\right)$ compressibility . . 22

3.8 Restoring incompressibility in the order $O\left(\mid \boldsymbol{\xi}^{2}\right)$ model . . . . . . . . . 24

3.9 The Euler-Lagrange equations for the LMM model . . . . . . . . . . 24

4 Physical interpretations of $u$ and $v$ as the Lagrangian and Eulerian $\begin{array}{ll}\text { mean fluid velocities } & 25\end{array}$

4.1 Stokes mean drift closure relation . . . . . . . . . . . . . . . . 26

$4.2 \quad$ An order $O\left(|\boldsymbol{\xi}|^{2}\right)$ model with $\operatorname{div}\langle\mathbf{U}\rangle^{E}=0 \ldots \ldots$. . . . . . . . 30

5 One point closure equations

$5.1 \quad$ Euler-Poincaré equation for the approximate $\langle L\rangle \ldots \ldots$. . . . . . 32

5.2 Relation to one point turbulence closure models . . . . . . . . . . . . 33

5.3 Comparison of VCHE or NS- $\alpha$ with LES and RANS models. . . . . 34

5.4 Comparison of VCHE or NS- $\alpha$ to Leray's equation . . . . . . . . . . 35

\begin{tabular}{|lll}
6 & Second moment closure equations & 37
\end{tabular}

6.1 Euler-Poincaré formulation . . . . . . . . . . . . . . . . 37

6.2 Momentum conservation - stress tensor formulation . . . . . . . . . 39

6.3 Momentum conservation for the order $O\left(|\boldsymbol{\xi}|^{2}\right)$ model . . . . . . . . . . 41

6.4 Kelvin circulation theorem for the Lagrangian mean model . . . . . 42

6.5 Vortex stretching equation for the Lagrangian mean model . . . . . 43

6.6 Energetics of the Lagrangian mean model . . . . . . . . . . . . . . . 43

7 Hamiltonian structure of the Lagrangian mean model 45

8 The 2D ideal LMM equations have no velocity Casimirs 46

\begin{tabular}{|lll}
\hline 9 & Relation to second moment turbulence closure models & 47
\end{tabular} 
10 Geophysical applications - adding rotation and stratification to the Lagrangian mean model

10.1 Formulation of Lagrangian mean Euler-Boussinesq (LMEB) equations 49

10.2 Passive scalar diffusion in the LMEB model. . . . . . . . . . . . . . 5 50

11 Lagrangian mean motion in fewer dimensions $\quad 51$

11.1 2D Lagrangian mean rotating shallow water (LMRSW) equations . . 51

11.2 One dimensional Lagrangian mean shallow water (LMSW) equations 53

11.3 The Lagrangian mean Riemann (LMR) equation . . . . . . . . . . . 54

12 Eulerian mean theory of advected fluctuations $\quad 55$

12.1 Kelvin circulation theorem for the Eulerian mean model . . . . . . . 59

12.2 Vortex stretching equation for the Eulerian mean model . . . . . . . 59

12.3 Energetics of the Eulerian mean mode . . . . . . . . . . . . . . . . 59

12.4 Momentum conservation - stress tensor formulation . . . . . . . . . 60

12.5 A second moment turbulence closure model for EMM . . . . . . . . 61

\begin{tabular}{|ll}
13 Geophysical applications of the Eulerian mean model & 61
\end{tabular}

13.1 Eulerian mean Euler-Boussinesq equations . . . . . . . . . . . . . . 61

13.22 D Eulerian mean rotating shallow water (EMRSW) . . . . . . . . . 63

13.3 1D Eulerian mean shallow water model . . . . . . . . . . . . . . . 64

13.4 The Eulerian mean Riemann (EMR) equation . . . . . . . . . . . . . 65

\begin{tabular}{ll}
\hline 14 Conclusions & 67
\end{tabular}

\section{Introduction}

We seek equations for the slow time dynamics of fluid motion that self consistently account for the effects of the variability upon the mean. In formulating such equations one must choose a suitable decomposition of the flow into its rapid and slowly varying components and determine a strategy for applying the corresponding averaging procedure. We consider Reynolds type decompositions of either the Lagrangian fluid trajectory, or the Eulerian fluid velocity. These decompositions lead respectively to either the Lagrangian mean (the time average following a fluid parcel), or the Eulerian mean (the time average at a fixed position). Our strategy in seeking self consistent slow time dynamics is to apply these decompositions and their corresponding averaging procedures to Hamilton's principle for an ideal incompressible fluid flow. The resulting Lagrangian mean and Eulerian mean equations are obtained in both cases by using the same Euler-Poincaré variational framework [1], [2]. Hence, these equations possess conservation laws for energy and momentum, as well as a Kelvin-Noether circulation theorem that establishes how the mean properties of the fluctuations affect the circulation of the mean flow.

Thus, we present two formulations of the mean equations we seek: a Lagrangian mean theory; and an Eulerian mean theory. It turns out these theories possess a 
certain duality. In particular, the Eulerian mean velocity appears as the momentum, or circulation velocity in the Lagrangian mean dynamics, and vice versa. The effect of the averaging in either case is to make the advection or transport velocity smoother than the momentum, or circulation velocity, via the inversion of a Helmholtz operator that relates the two velocities. The length scale that appears in this Helmholtz operator is the covariance of the fluctuations, which has its own dynamics in each case. Thus, the temporal averaging in Hamilton's principle leads to a dynamical spatial filtering in the resulting equations of motion. This is the main point of the paper. The two formulations we present here complement each other and provide a flexible unified basis for further investigation and analysis of the effects of fluctuations on mean fluid dynamics.

We begin by introducing new equations that describe the Lagrangian mean effects of advected fluctuations in 3D incompressible fluid motion. The results include a new second moment closure model for 3D fluid turbulence. This model describes dynamically self-consistent interaction between a Lagrangian mean flow and a distribution of advecting rapid (or random) fluctuations described in slow time (or statistically) by their Lagrangian mean covariance tensor. Its derivation combines the Euler-Poincaré theory of fluid dynamics, the Taylor hypothesis for advection of the fluctuations and the Reynolds decomposition of the Lagrangian fluid trajectory. We also consider the effects of rotation and stratification in this model, for the sake of its potential geophysical applications. To help develop intuition about the solution behavior of these Lagrangian mean models we discuss 1D and 2D subcases, as well. Then, we compare the Lagrangian mean models with their Eulerian mean counterparts and emphasize the duality between them.

\subsection{Lagrangian mean equations}

This Section introduces a new self-consistent dynamical model that describes the Lagrangian mean effects of advected fluctuations on 3D incompressible stratified fluid motion in a rotating frame. This model is based on the Lagrangian fluid description of two standard assumptions: (1) Reynolds decomposition of the Lagrangian fluid trajectory; and (2) the Taylor hypothesis, that rapid fluctuations are advected by the mean flow. We substitute these two assumptions into Hamilton's principle for the Euler equations of an ideal incompressible fluid, and apply the Lagrangian mean before taking variations. Dissipation is then introduced in the traditional semi-empirical fashion. The results include a new second moment closure model for $3 \mathrm{D}$ fluid turbulence. The latter is a development of the one point closure model of Chen et al. [3]- [5], based on the viscous Camassa-Holm equations (VCHE), also known as the Navier-Stokes alpha model. The Lagrangian mean Euler-Boussinesq (LMEB) model for a stratified incompressible fluid in a rotating reference frame is given by the equations, 


$$
\begin{aligned}
& \frac{d}{d t} \mathbf{v}-\mathbf{u} \times \operatorname{curl} \mathbf{R}(\mathbf{x})+\nabla p+g b \hat{\mathbf{z}}=\nu \tilde{\Delta} \mathbf{v}, \quad \text { with } \quad \boldsymbol{\nabla} \cdot \mathbf{u}=0 \\
& \text { where } \frac{d}{d t} \equiv\left(\frac{\partial}{\partial t}+\mathbf{u} \cdot \boldsymbol{\nabla}\right), \quad \mathbf{v} \equiv(1-\tilde{\Delta}) \mathbf{u}, \quad \tilde{\Delta} \equiv \boldsymbol{\nabla} \cdot\langle\boldsymbol{\xi} \boldsymbol{\xi}\rangle \cdot \boldsymbol{\nabla} \\
& \text { and } \quad \frac{d b}{d t}=\left(\boldsymbol{\nabla} \cdot \boldsymbol{\kappa}_{S} \cdot \boldsymbol{\nabla}\right) b \quad \text { with } \quad 2 \boldsymbol{\kappa}_{S} \equiv \frac{d}{d t}\langle\boldsymbol{\xi} \boldsymbol{\xi}\rangle=\langle\boldsymbol{\xi} \boldsymbol{\xi}\rangle \cdot \boldsymbol{\nabla} \mathbf{u}+\boldsymbol{\nabla} \mathbf{u}^{\mathrm{T}} \cdot\langle\boldsymbol{\xi} \boldsymbol{\xi}\rangle
\end{aligned}
$$

These LMEB equations include the standard Euler-Boussinesq (EB) equations as an invariant subsystem, for which $\langle\boldsymbol{\xi} \xi\rangle=0$. The LMEB introduce the additional dynamics of the covariance $\langle\boldsymbol{\xi} \xi\rangle$ of the rapid fluid parcel displacement fluctuations into the metric of the dynamical Helmholtz operator, $1-\tilde{\Delta}$. Because of the advective nature of the $\langle\boldsymbol{\xi} \boldsymbol{\xi}\rangle$ dynamics, this Helmholtz operator commutes with the advective time derivative, $d / d t$ for the Lagrangian mean velocity.

The LMEB model introduces two different fluid velocities into the average description. The velocities in the LMEB equations (1.1) - (1.2) are $\mathbf{u}$, defined to be the Lagrangian mean velocity; and $\mathbf{v}=(1-\tilde{\Delta}) \mathbf{u}$, found later to be the Eulerian mean velocity, to order $o\left(|\boldsymbol{\xi}|^{2}\right)$. It is also useful to think of the velocity $\mathbf{u}$ as the particle, or transport velocity, while the velocity $\mathbf{v}$ is the flow, or circulation velocity. Because of the relation $\mathbf{v}=(1-\tilde{\Delta}) \mathbf{u}$, the transport velocity $\mathbf{u}$ is smoother than the flow, or circulation velocity $\mathbf{v}$, by the inversion of the dynamical Helmholtz operator, $(1-\tilde{\Delta})$. That is, in the advective time derivative $d / d t=(\partial / \partial t+\mathbf{u} \cdot \nabla)$, we have $\mathbf{u}=(1-\tilde{\Delta})^{-1} \mathbf{v}$. The difference $\mathbf{u}-\mathbf{v}=\tilde{\Delta} \mathbf{u}$ is the Stokes mean drift velocity due to the presence of the rapid fluctuations with Lagrangian mean covariance $\langle\boldsymbol{\xi} \xi\rangle$. The interpretation of its effects will be a recurring theme in this paper.

The symmetric tensor $\boldsymbol{\kappa}_{S}=\frac{1}{2} d\langle\boldsymbol{\xi} \boldsymbol{\xi}\rangle / d t$ is the Taylor diffusivity tensor (defined here without including any antisymmetric corrections due to rotation). The displacement fluctuation $\boldsymbol{\xi}$ is defined by $\boldsymbol{\xi}=\mathbf{X}^{\xi}-\mathbf{X}$ with $\mathbf{X}=\left\langle\mathbf{X}^{\xi}\right\rangle$ for an averaging process (the Lagrangian mean) taken at fixed Lagrangian label a and denoted $\langle\cdot\rangle$ with, e.g.,

$$
\mathbf{X}(\mathbf{a}, t)=\left\langle\mathbf{X}^{\xi}(\mathbf{a}, t ; \omega)\right\rangle \equiv \lim _{T \rightarrow \infty} \frac{1}{T} \int_{-T}^{T} \mathbf{X}^{\xi}(\mathbf{a}, t ; \omega) d \omega
$$

Thus, a displacement fluctuation satisfying $\langle\boldsymbol{\xi}\rangle=0$ is given by

$$
\boldsymbol{\xi}(\mathbf{X}, t ; \omega) \equiv \mathbf{X}^{\xi}(\mathbf{a}, t ; \omega)-\mathbf{X}(\mathbf{a}, t) .
$$

This displacement fluctuation has covariance

$$
\langle\xi \xi\rangle \equiv\left\langle\mathbf{X}^{\xi} \mathbf{X}^{\xi}\right\rangle-\mathbf{X X}
$$

Here, $\mathbf{X}^{\xi}(\mathbf{a}, t ; \omega)$ is the spatial trajectory of a fluid parcel with Lagrangian label a that is undergoing EB dynamics. We assume this motion depends on both a slow time $t$ and a rapid (or random) time variation $\omega$. In taking the Lagrangian mean of this spatial trajectory, we average at constant fluid label a over its rapid variation in $\omega$ during a time interval $T$ that is long (denoted $\lim _{T \rightarrow \infty}$ ) compared 
to the rapid time scale for variation in $\omega$, but during which the slow time variation in $t$ may be regarded as fixed. In particular, we shall assume that the result of the averaging operation $\langle\cdot\rangle$ is independent of the magnitude of the time interval $T$. Thus, in equation (1.4) one may think of the Lagrangian trajectory $\mathbf{X}^{\xi}(\mathbf{a}, t ; \omega)$ as following the original EB dynamics in a flow regime in which a separation of time scales into slow $(t)$ and fast $(\omega)$ is possible, and think of $\mathbf{X}(\mathbf{a}, t)$ as following the approximate slow time dynamics determined by the LMEB equations. We shall see that $p$ is the Lagrangian mean pressure. The other notation in (1.1) and (1.2) is standard for fluid dynamics: $b$ is buoyancy; $\nu$ is kinematic viscosity; $g$ is the constant acceleration of gravity; and $\operatorname{curl} \mathbf{R}=2 \boldsymbol{\Omega}(\mathbf{x})$ is the Coriolis parameter, which may depend on position. The boundary conditions of the dissipative LMEB model are

$$
\mathbf{v}=0, \quad \mathbf{u}=0 \quad \text { and } \quad\langle\boldsymbol{\xi} \boldsymbol{\xi}\rangle \cdot \hat{\boldsymbol{n}}=0 \quad \text { on a fixed boundary }
$$

When dissipation, rotation and stratification are absent, the LMEB model in (1.1) - (1.2) reduces to the ideal Lagrangian mean motion (LMM) equations:

$$
\begin{aligned}
& \frac{d}{d t} \mathbf{v}+\nabla p=0, \quad \text { with } \quad \boldsymbol{\nabla} \cdot \mathbf{u}=0, \\
& \text { where } \frac{d}{d t} \equiv\left(\frac{\partial}{\partial t}+\mathbf{u} \cdot \boldsymbol{\nabla}\right), \quad \mathbf{v} \equiv(1-\tilde{\Delta}) \mathbf{u}, \quad \tilde{\Delta} \equiv \boldsymbol{\nabla} \cdot\langle\boldsymbol{\xi} \xi \cdot \nabla, \\
& \text { and } \frac{d}{d t}\langle\boldsymbol{\xi} \boldsymbol{\xi}\rangle=\langle\boldsymbol{\xi} \boldsymbol{\xi}\rangle \cdot \boldsymbol{\nabla} \mathbf{u}+\boldsymbol{\nabla} \mathbf{u}^{\mathrm{T}} \cdot\langle\boldsymbol{\xi} \boldsymbol{\xi}\rangle .
\end{aligned}
$$

The boundary conditions for this ideal LMM model are:

$$
\mathbf{v} \cdot \hat{\boldsymbol{n}}=0, \quad \mathbf{u}=0, \quad \text { and } \quad\langle\boldsymbol{\xi} \boldsymbol{\xi}\rangle \cdot \hat{\boldsymbol{n}}=0 \quad \text { on a fixed boundary }
$$

The LMM motion equation (1.7) may be rewritten equivalently as

$$
\left(\frac{\partial}{\partial t}+\mathbf{v} \cdot \boldsymbol{\nabla}\right) \mathbf{v}+\underbrace{(\mathbf{u}-\mathbf{v}) \cdot \boldsymbol{\nabla} \mathbf{v}}_{\text {Stokes transport }}+\nabla p=0 .
$$

Thus, perhaps not unexpectedly, the Stokes mean drift velocity $\mathbf{u}-\mathbf{v}=\tilde{\Delta} \mathbf{u}$ contributes an additional transport term in this motion equation for the Eulerian mean velocity $\mathbf{v}$. On the invariant manifold $\langle\boldsymbol{\xi} \boldsymbol{\xi}\rangle=0$ the LMEB and LMM equation sets recover their original forms.

The ideal LMM model preserves the total kinetic energy,

$$
E=\frac{1}{2} \int d^{3} x\left(|\mathbf{u}|^{2}+\left\langle\xi^{k} \xi^{l}\right\rangle \mathbf{u}_{, k} \cdot \mathbf{u}_{, l}\right)=\frac{1}{2} \int d^{3} x \mathbf{u} \cdot \mathbf{v}
$$

in which the Lagrangian covariance of the fluctuations couples to the gradients of the Lagrangian mean velocity. (Throughout the paper, we sum on repeated indices.) Conservation of this energy provides $L^{2}$ control on $|\nabla \mathbf{u}|$, provided $\langle\boldsymbol{\xi} \boldsymbol{\xi}\rangle$ is bounded away from zero. In fact, for incompressible Lagrangian mean velocity $\mathbf{u}$, the determinant $\operatorname{det}\langle\boldsymbol{\xi} \boldsymbol{\xi}\rangle$ is conserved on fluid parcels. Hence, the covariance 
does remain bounded away from zero, if it is initially so. This statement still holds when order $O\left(|\boldsymbol{\xi}|^{2}\right)$ compressibility is allowed. The ideal LMM model also conserves the domain integrated momentum, $\int \mathbf{v} d^{3} x$. (With boundary conditions (1.9), this conserved momentum is also equal to $\int \mathbf{u} d^{3} x$.)

The ideal LMM model possesses a Kelvin-Noether circulation theorem showing how the Stokes drift velocity generates circulation of v. Namely,

$$
\frac{d}{d t} \oint_{\gamma(\mathbf{u})} \mathbf{v} \cdot d \mathbf{x}=-\iint_{S(\mathbf{u})}\left[\boldsymbol{\nabla} \tilde{\Delta} u_{j} \times \nabla u^{j}\right] \cdot d \mathbf{S}
$$

where the closed curve $\gamma(\mathbf{u})$ moves with the Lagrangian mean fluid velocity $\mathbf{u}$ and is the boundary of the surface $S(\mathbf{u})$. By virtue of the operator commutation relation

$$
\frac{d}{d t} \tilde{\Delta}-\tilde{\Delta} \frac{d}{d t} \equiv\left[\frac{d}{d t}, \tilde{\Delta}\right]=0
$$

we may also express the ideal LMM motion equation (1.7) in its alternative LMM form,

$$
\frac{\partial \mathbf{u}}{\partial t}+\mathbf{u} \cdot \nabla \mathbf{u}=-(1-\tilde{\Delta})^{-1} \nabla p, \quad \nabla \cdot \mathbf{u}=0
$$

In this form, the effect of the advected fluctuations is to smooth the pressure gradient in an adaptive fashion depending on the velocity shear through the evolution of $\tilde{\Delta}$. The divergence of equation (1.14) yields an elliptic equation for the Lagrangian mean pressure, $p$. The Kelvin-Noether circulation theorem corresponding to the form (1.14) of the LMM motion equation is

$$
\frac{d}{d t} \oint_{\gamma(\mathbf{u})} \mathbf{u} \cdot d \mathbf{x}=-\iint_{S(\mathbf{u})}\left[\boldsymbol{\nabla} \times(1-\tilde{\Delta})^{-1} \nabla p\right] \cdot d \mathbf{S},
$$

which represents the circulation dynamics of $\mathbf{u}$, rather than $\mathbf{v}$.

Thus, the presence of the fluctuations with Lagrangian mean covariance $\langle\boldsymbol{\xi} \boldsymbol{\xi}\rangle$ in the ideal LMM equations has five main effects, relative to the Euler equations:

1. it smoothes the Lagrangian mean (transport) velocity $\mathbf{u}$ relative to the Eulerian mean (momentum) velocity $\mathbf{v}$;

2. it introduces an additional transport of the Eulerian mean velocity $\mathbf{v}$ by the Stokes drift velocity $\mathbf{u}-\mathbf{v}$;

3. it controls $\|\nabla \mathbf{u}\|_{2}$ in the $L^{2}$ norm via energy conservation (or energy dissipation, when viscosity is included);

4. it creates circulation of both Eulerian mean velocity $\mathbf{v}$ and Lagrangian mean fluid velocity $\mathbf{u}$ around closed loops advecting with the fluid parcels; and

5. it smoothes the pressure gradient in an adaptive fashion depending on the evolution of the fluctuation covariance $\langle\boldsymbol{\xi} \boldsymbol{\xi}\rangle$, driven by the velocity shear $\boldsymbol{\nabla} \mathbf{u}$. 
Most of this paper is devoted to deriving the LMM and LMEB equations by approximating Taylor series expansions and averaging over the rapid time dependence in Hamilton's principle for the Euler equations of an ideal incompressible fluid. We average over "fast time" at fixed Lagrangian fluid label in Hamilton's principle for the Euler equations. This averaging over the rapid "microscopic" fluid motions allows us to extract an approximate Lagrangian mean Hamilton's principle whose Euler-Lagrange equations describe the remaining slow "macroscopic" fluid motions. After a discussion of these Euler-Lagrange equations from the viewpoint of the Lagrangian fluid parcel description, we use the Euler-Poincaré theory of Holm, Marsden and Ratiu [1], [2], to develop and analyze the ideal LMM equations (1.7) (1.8) in the Eulerian description. We then add rotation and stratification to derive the ideal LMEB equations. Finally, we introduce dissipation according to the traditional semi-empirical reasoning by requiring that the energy dissipation rate be negative definite. This both adds the fluctuation-dependent viscosity in the motion equation and introduces the dynamics for the Taylor diffusivity tensor $\boldsymbol{\kappa}_{S}$ into the buoyancy equation.

A second moment Lagrangian mean turbulence model is obtained by adding phenomenological viscosity $\nu \tilde{\Delta} \mathbf{v}$ and forcing $\mathbf{F}$ to the ideal LMM motion equation (1.7), so that,

$$
\left(\frac{\partial}{\partial t}+\mathbf{u} \cdot \boldsymbol{\nabla}\right) \mathbf{v}+\nabla p=\nu \tilde{\Delta} \mathbf{v}+\mathbf{F}, \quad \boldsymbol{\nabla} \cdot \mathbf{u}=0
$$

with viscous boundary conditions (1.6). Note that the Lagrangian mean fluctuation covariance appears in the dissipation operator $\tilde{\Delta}$. In the absence of the forcing $\mathbf{F}$, this viscous LMM turbulence model dissipates the energy $E$ in equation (1.11) according to

$$
\frac{d E}{d t}=-\nu \int d^{3} x\left[\operatorname{tr}\left(\boldsymbol{\nabla} \mathbf{u}^{T} \cdot\langle\boldsymbol{\xi} \boldsymbol{\xi}\rangle \cdot \nabla \mathbf{u}\right)+\tilde{\Delta} \mathbf{u} \cdot \tilde{\Delta} \mathbf{u}\right]
$$

This strictly negative energy dissipation rate is the reason for adding viscosity with $\tilde{\Delta}$, instead of using the ordinary Laplacian operator.

The generalization of the Lagrangian mean motion model to Riemannian manifolds - to make it applicable in any coordinate system and to elucidate its intrinsic geometrical structure - will be discussed elsewhere [6]. We will also defer the derivation of the isopycnal and hydrostatic versions of this model to another time and place [7].

\subsection{Eulerian mean equations}

We shall also present a parallel development of these Lagrangian mean results, but applied to Eulerian mean models. The Eulerian mean Euler-Boussinesq $(\boldsymbol{E} \boldsymbol{M E B})$ equations are given by 


$$
\begin{aligned}
& \frac{d}{d t} \mathbf{V}+V_{j} \nabla \bar{U}^{j}-\overline{\mathbf{U}} \times \operatorname{curl} \mathbf{R}(\mathbf{x})+\nabla P_{t o t}^{E} \\
& +g b \hat{\mathbf{z}}+\frac{1}{2}\left(\overline{\mathbf{U}}_{, k} \cdot \overline{\mathbf{U}}_{, l}\right) \boldsymbol{\nabla}\left\langle\zeta^{k} \zeta^{l}\right\rangle^{E}=\nu \tilde{\Delta}^{E} \mathbf{V}, \\
& \frac{d b}{d t}=\kappa \tilde{\Delta}^{E} b, \quad \frac{d}{d t}\langle\zeta \zeta\rangle^{E}=0, \quad P_{t o t}^{E} \equiv P-\frac{1}{2}|\overline{\mathbf{U}}|^{2}-\frac{1}{2}\left\langle\zeta^{k} \zeta^{l}\right\rangle^{E}\left(\overline{\mathbf{U}}_{, k} \cdot \overline{\mathbf{U}}_{, l}\right), \\
& \text { and } \quad \nabla \cdot \overline{\mathbf{U}}=0, \quad \text { where } \quad \overline{\mathbf{U}} \equiv\langle\mathbf{U}\rangle^{E} \text {. }
\end{aligned}
$$

Here $P$ is the Eulerian mean pressure, and $\nu$ and $\kappa$ are constants representing viscosity and diffusivity, respectively. The boundary conditions for this viscous EMEB model are:

$$
\mathbf{V}=0, \quad \overline{\mathbf{U}}=0, \quad \text { and }\langle\zeta \zeta\rangle^{E} \cdot \hat{\boldsymbol{n}}=0 \quad \text { on a fixed boundary. }
$$

These equations correspond to the same level of approximation as those for LMEB, but they are based on the Eulerian mean velocity $\overline{\mathbf{U}}$, appearing in the Reynolds fluid velocity decomposition,

$$
\mathbf{U}(\mathbf{x}, t ; \omega) \equiv \overline{\mathbf{U}}(\mathbf{x}, t)+\mathbf{U}^{\prime}(\mathbf{x}, t ; \omega) .
$$

The Eulerian mean averaging process at fixed position $\mathrm{x}$ is denoted $\langle\cdot\rangle^{E}$ with, e.g.,

$$
\overline{\mathbf{U}}(\mathbf{x}, t)=\langle\mathbf{U}(\mathbf{x}, t ; \omega)\rangle^{E} \equiv \lim _{T \rightarrow \infty} \frac{1}{T} \int_{-T}^{T} \mathbf{U}(\mathbf{x}, t ; \omega) d \omega
$$

We shall show that the Eulerian velocity fluctuation $\mathbf{U}^{\prime}$ is related to the Eulerian displacement fluctuation $\zeta$ by

$$
0=\zeta \cdot \nabla \overline{\mathbf{U}}+\mathbf{U}^{\prime}(\mathbf{x}, t ; \omega) .
$$

Consequently, the Eulerian mean kinetic energy due to the velocity fluctuation satisfies

$$
\left\langle\left|\mathbf{U}^{\prime}\right|^{2}\right\rangle^{E}=\left\langle\zeta^{k} \zeta^{l}\right\rangle^{E} \overline{\mathbf{U}}_{, k} \cdot \overline{\mathbf{U}}_{, l} .
$$

Note that the advection of the Eulerian mean displacement fluctuation covariance $\langle\zeta \zeta\rangle^{E}$ by the Eulerian mean velocity $\overline{\mathbf{U}}$ is componentwise: $d\langle\zeta \zeta\rangle^{E} / d t=0$, so each component of this symmetric tensor is carried along with the Eulerian mean flow as if it were a scalar function. Moreover, we shall show that the momentum $\mathbf{V}$ appearing in the Euler-Poincaré formulation of these EMEB equations is in fact the Lagrangian mean velocity for this theory. This duality between Lagrangian mean and Eulerian mean theories is another theme of the present paper.

In the absence of dissipation, rotation and stratification, the EMEB model in (1.18) - (1.19) reduces to the ideal Eulerian mean motion (EMM) equations: 


$$
\begin{aligned}
& \left(\frac{\partial}{\partial t}+\overline{\mathbf{U}} \cdot \boldsymbol{\nabla}\right) \mathbf{V}+V_{j} \boldsymbol{\nabla} \bar{U}^{j}+\nabla P_{t o t}^{E}+\frac{1}{2}\left(\overline{\mathbf{U}}_{, k} \cdot \overline{\mathbf{U}}_{, l}\right) \boldsymbol{\nabla}\left\langle\zeta^{k} \zeta^{l}\right\rangle^{E}=0 \\
& \text { where } \quad \mathbf{V} \equiv\left(1-\tilde{\Delta}^{E}\right) \overline{\mathbf{U}}, \quad \tilde{\Delta}^{E} \equiv \boldsymbol{\nabla} \cdot\langle\boldsymbol{\zeta} \boldsymbol{\zeta}\rangle^{E} \cdot \boldsymbol{\nabla} \\
& \boldsymbol{\nabla} \cdot \overline{\mathbf{U}}=0, \quad \text { and } \quad\left(\frac{\partial}{\partial t}+\overline{\mathbf{U}} \cdot \boldsymbol{\nabla}\right)\langle\boldsymbol{\zeta} \zeta\rangle^{E}=0 .
\end{aligned}
$$

The boundary conditions for this ideal EMM model are:

$$
\mathbf{V} \cdot \hat{\boldsymbol{n}}=0, \quad \overline{\mathbf{U}}=0, \quad \text { and } \quad\langle\boldsymbol{\zeta} \boldsymbol{\zeta}\rangle \cdot \hat{\boldsymbol{n}}=0 \quad \text { on a fixed boundary }
$$

The EMM motion equation (1.25) may be rewritten equivalently as

$$
\left(\frac{\partial}{\partial t}+\mathbf{V} \cdot \boldsymbol{\nabla}\right) \mathbf{V}+\underbrace{(\mathbf{V}-\overline{\mathbf{U}}) \times \operatorname{curl} \mathbf{V}}+\nabla P-\frac{1}{2}\left\langle\zeta^{k} \zeta^{l}\right\rangle^{E} \boldsymbol{\nabla}\left(\overline{\mathbf{U}}_{, k} \cdot \overline{\mathbf{U}}_{, l}\right)=0 .
$$

\section{Stokes vortex force}

Thus, the Stokes mean drift velocity $\mathbf{V}-\overline{\mathbf{U}}=\tilde{\Delta}^{E} \overline{\mathbf{U}}$ contributes an additional vortex force in this motion equation for the Lagrangian mean fluid velocity $\mathbf{V}$.

On the invariant manifold $\boldsymbol{\nabla}\langle\boldsymbol{\zeta} \zeta\rangle^{E}=0$ the EMM equation set reduces to the 3D ideal Camassa-Holm model [1], [2]. The Eulerian mean system (1.25) - (1.26) recovers the 3D ideal Camassa-Holm model as an invariant subsystem for $\left\langle\zeta^{k} \zeta^{l}\right\rangle^{E}=$ $\alpha^{2} \delta^{k l}$, with $\alpha$ a constant length scale.

The ideal EMM model preserves the total kinetic energy,

$$
E=\frac{1}{2} \int d^{3} x\left(|\overline{\mathbf{U}}|^{2}+\left\langle\zeta^{k} \zeta^{l}\right\rangle^{E} \overline{\mathbf{U}}_{, k} \cdot \overline{\mathbf{U}}_{, l}\right)=\frac{1}{2} \int d^{3} x \overline{\mathbf{U}} \cdot \mathbf{V}
$$

in which the Eulerian covariance of the fluctuations couples to the gradients of the Eulerian mean velocity. Conservation of this energy provides $L^{2}$ control on $|\nabla \overline{\mathbf{U}}|$, provided $\langle\xi \xi\rangle^{E}$ is bounded away from zero, which it will be, if it is initially so. The ideal EMM model also conserves the domain integrated momentum, $\int \mathbf{V} d^{3} x$. (With boundary conditions (1.27), this conserved momentum is also equal to $\int \overline{\mathbf{U}} d^{3} x$.)

The ideal EMM model possesses a Kelvin-Noether circulation theorem showing how the Stokes drift velocity generates circulation of V. Namely,

$$
\frac{d}{d t} \oint_{\gamma(\overline{\mathbf{U}})} \mathbf{V} \cdot d \mathbf{x}=-\frac{1}{2} \iint_{S(\overline{\mathbf{U}})}\left[\boldsymbol{\nabla}\left(\overline{\mathbf{U}}_{, k} \cdot \overline{\mathbf{U}}_{, l}\right) \times \nabla\left\langle\zeta^{k} \zeta^{l}\right\rangle^{E}\right] \cdot d \mathbf{S},
$$

where the closed curve $\gamma(\overline{\mathbf{U}})$ moves with the Eulerian mean fluid velocity $\overline{\mathbf{U}}$ and is the boundary of the surface $S(\overline{\mathbf{U}})$.

Thus, the presence of the fluctuations with Eulerian mean covariance $\langle\zeta \zeta\rangle^{E}$ in the ideal EMM equations has four main effects, relative to the Euler equations:

1. it smoothes the Eulerian mean (transport) velocity $\overline{\mathbf{U}}$ relative to the Lagrangian mean (momentum) velocity $\mathbf{V}$;

2. it introduces an additional vortex force into the Lagrangian mean velocity equation due to the Stokes drift velocity $\overline{\mathbf{U}}-\mathbf{V}=\tilde{\Delta}^{E} \overline{\mathbf{U}}$; 
3. it controls $\|\boldsymbol{\nabla} \overline{\mathbf{U}}\|_{2}$ in the $L^{2}$ norm via energy conservation (or energy dissipation, when viscosity is included); and

4. it creates circulation of Lagrangian mean velocity $\mathbf{V}$ around closed loops advecting with the Eulerian mean velocity $\overline{\mathbf{U}}$.

A second moment Eulerian mean turbulence model is obtained by adding phenomenological viscosity $\nu \tilde{\Delta}^{E} \mathbf{V}$ and forcing $\mathbf{F}$ to the ideal EMM motion equation (1.25), so that,

$$
\begin{aligned}
\left(\frac{\partial}{\partial t}+\overline{\mathbf{U}} \cdot \boldsymbol{\nabla}\right) \mathbf{V}+V_{j} \nabla \bar{U}^{j} & +\nabla P_{t o t}^{E}+\frac{1}{2}\left(\overline{\mathbf{U}}_{, k} \cdot \overline{\mathbf{U}}_{, l}\right) \nabla\left\langle\zeta^{k} \zeta^{l}\right\rangle^{E} \\
& =\nu \tilde{\Delta}^{E} \mathbf{V}+\mathbf{F}, \quad \text { where } \quad \nabla \cdot \overline{\mathbf{U}}=0,
\end{aligned}
$$

with viscous boundary conditions $(1.20)$. Note that the Eulerian mean fluctuation covariance appears in the dissipation operator $\tilde{\Delta}^{E}$. In the absence of the forcing $\mathbf{F}$, this viscous EMM turbulence model dissipates the energy $E$ in equation (1.29) according to

$$
\frac{d E}{d t}=-\nu \int d^{3} x\left[\operatorname{tr}\left(\nabla \overline{\mathbf{U}}^{T} \cdot\langle\zeta \zeta\rangle^{E} \cdot \nabla \overline{\mathbf{U}}\right)+\tilde{\Delta}^{E} \overline{\mathbf{U}} \cdot \tilde{\Delta}^{E} \overline{\mathbf{U}}\right] .
$$

This energy dissipation law justifies adding viscosity with $\tilde{\Delta}^{E}$, instead of using the ordinary Laplacian operator.

\subsection{Outline of the paper}

In Section 2, we shall recall the Euler-Poincaré equations (2.11) in the context of the ideal Euler fluid equations. In Section 3, we shall introduce into Hamilton's principle for Euler's equations the Reynolds decomposition of a Lagrangian fluid trajectory as the sum of its mean and fluctuating parts. In the remainder of Section 3, we shall: substitute this decomposition into the Lagrangian for Euler's equations; transform the resulting rapid (or random) Lagrangian $L(\omega)$ in equation (3.4) into the Eulerian description; make an approximation of it based on truncating a Taylor expansion; take its Lagrangian mean, denoted $\langle L\rangle$ in equation (3.34); and finally use the EulerPoincaré theorem to obtain approximate Lagrangian mean motion equations at order $O\left(|\boldsymbol{\xi}|^{2}\right)$. In Section $⿴$ we shall provide the physical interpretations of the quantities arising during this modeling procedure and compare the resulting equations with other models. In Section 5 we shall discuss both ideal and viscous one point closure models based on the Euler-Poincaré equation. We shall also compare these one point closure models with Reynolds-averaged Navier-Stokes (RANS) models, Large Eddy Simulation (LES) models, and the Leray regularization of the Navier-Stokes equations.

In Section 6 we shall augment the Euler-Poincaré equation in (6.7) to include dynamically varying correlations of the rapid/random fluctuations and use this Lagrangian mean fluid theory to derive a new approximate motion equation for the slow evolution of the Lagrangian mean fluid velocity in Eulerian coordinates. This 
is the basis for the Eulerian analysis of the Lagrangian mean motion (LMM) model. The LMM equations in (6.17) - (6.20) will include self-consistent forces caused by the correlations of the rapid/random fluctuations and expressed in terms of their Lagrangian mean covariance tensor, $\langle\boldsymbol{\xi} \boldsymbol{\xi}\rangle$. The approximate dynamics of the covariance tensor $\langle\boldsymbol{\xi} \boldsymbol{\xi}\rangle$ itself will also be determined, thereby producing a two point, or second moment, Lagrangian mean closure of Euler's ideal incompressible fluid equations. Several properties of this ideal second moment closure model are also derived in Section 6, including its conservation laws for momentum and energy, and its Kelvin-Noether circulation theorem, which are all inherited from the Euler-Poincaré formulation. We note that the Lagrangian mean effects of the rapid fluctuations are purely dispersive at this stage and, thus, are energy conserving.

The Lie-Poisson Hamiltonian structure of the ideal LMM model is given in Section 7 . Its $2 \mathrm{D}$ behavior is discussed briefly in Section 8. Upon adding viscosity in Section 9, we shall introduce a second moment Lagrangian mean closure of the Navier-Stokes equations corresponding to the LMM model and compare this closure with the Reynolds averaged Navier-Stokes equations.

In Section 10, we shall discuss geophysical applications of these ideas and use our approach via the Euler-Poincaré theory to derive the Lagrangian mean EulerBoussinesq (LMEB) equation set (10.2) - (10.3) for the Lagrangian mean motion of a rotating stratified turbulent incompressible fluid. Section 11 considers lower dimensional subcases in one and two dimensions, in an effort to help develop intuition about the solution behavior of these Lagrangian mean models.

Section 12 studies the Eulerian mean counterpart of the LMM model and emphasizes the duality and parallel mathematical structures shared in the two approaches through their formulations as Euler-Poincaré equations. Section 13 adds rotation and stratification to the Eulerian mean model, then discusses some of its aspects in fewer dimensions. Section 14 contains a summary and conclusions.

\section{Review of Hamilton's principle and Euler-Poincaré equations for ideal fluids}

Consider the Lagrangian $L$ comprised of fluid kinetic energy with volume preservation imposed by a Lagrange multiplier $P$ (the pressure),

$$
L(\mathbf{X}, \dot{\mathbf{X}})=\int d^{3} a\left\{\frac{1}{2}|\dot{\mathbf{X}}(\mathbf{a}, t)|^{2}+P(\mathbf{X}(\mathbf{a}, t), t)\left(\operatorname{det}\left(\mathbf{X}_{a}^{\prime}\right)(\mathbf{a}, t)-1\right)\right\}
$$

Here, $\mathbf{X}(\mathbf{a}, t)$ is the Lagrangian fluid trajectory: that is, $\mathbf{x}=\mathbf{X}(\mathbf{a}, t)$ is the current position of the material point starting at initial position a at time $t=0$. We denote the derivatives of the function $\mathbf{X}(\mathbf{a}, t)$ by $\dot{\mathbf{X}}=\partial \mathbf{X} / \partial t$ and $\mathbf{X}_{a}^{\prime}=\partial \mathbf{X} / \partial \mathbf{a}$. After a brief calculation, Hamilton's principle,

$$
\delta \int d t L=0,
$$


for the Lagrangian $L(\mathbf{X}, \dot{\mathbf{X}})$ yields the following Euler-Lagrange equations,

$$
\delta \mathbf{X}:\left.\quad \frac{\partial}{\partial t}\right|_{\mathbf{a}} \frac{\delta L}{\delta \dot{\mathbf{X}}}-\frac{\delta L}{\delta \mathbf{X}}=0,
$$

given explicitly by (dropping boundary and endpoint contributions),

$$
\begin{array}{ll}
\delta \mathbf{X}: & \ddot{\mathbf{X}}+\operatorname{det}\left(\mathbf{X}_{a}^{\prime}\right) \frac{\partial P}{\partial \mathbf{X}}=0 \\
\delta P: & \operatorname{det}\left(\mathbf{X}_{a}^{\prime}\right)=1
\end{array}
$$

These are Euler's equations for the incompressible motion of an ideal fluid in the Lagrangian description.

We obtain the Eulerian description of this motion by defining the Eulerian fluid velocity $\mathbf{U}(\mathbf{x}, t)$ and volume element $D(\mathbf{x}, t)$ via the basic kinematic relations,

$$
\mathbf{U}(\mathbf{x}, t)=\dot{\mathbf{X}}(\mathbf{a}, t) \quad \text { and } \quad D(\mathbf{x}, t)=\left(\operatorname{det} \mathbf{X}_{a}^{\prime}(\mathbf{a}, t)\right)^{-1} \quad \text { at } \quad \mathbf{x}=\mathbf{X}(\mathbf{a}, t) .
$$

The volume element $D$ satisfies the continuity equation,

$$
\frac{\partial D}{\partial t}+\nabla \cdot(D \mathbf{U})=0
$$

The volume element $D(\mathbf{x}, t)$ is an advected quantity, (sometimes called "frozenin"). By this, we mean a quantity that is expressible purely in terms of Lagrangian labels; so that it is invariant along the Lagrangian mean fluid trajectory, or equivalently, satisfies a certain Lie-derivative relation [1]. For example, the volume element $D(\mathbf{x}, t)$ is expressible as the Jacobian for the transformation from Lagrangian to Eulerian coordinates. Thus, the volume element satisfies

$$
D d^{3} x=d^{3} a,
$$

and the continuity equation (2.7) is implied by the corresponding invariance relation for such an advected quantity, in this case,

$$
0=\left.\frac{\partial}{\partial t}\right|_{\mathbf{a}}\left(d^{3} a\right)=\left(\frac{\partial}{\partial t}+£_{\mathbf{U}}\right)\left(D d^{3} x\right)=\left(\frac{\partial D}{\partial t}+\nabla \cdot(D \mathbf{U})\right)\left(d^{3} x\right),
$$

where $£_{\mathbf{U}}$ denotes Lie derivative with respect to the vector field $\mathbf{U}(\mathbf{x}, t)$, the Eulerian fluid velocity. The basic relations (2.6) allow one to transform the Lagrangian (2.1) into Eulerian variables as

$$
L=\int d^{3} x\left\{\frac{D}{2}|\mathbf{U}(\mathbf{x}, t)|^{2}+P(\mathbf{x}, t)(1-D(\mathbf{x}, t))\right\} .
$$

Hamilton's principle $\delta \int d t L=0$ for a Lagrangian $L(\mathbf{U}, D)$ defined in this way yields the following Euler-Poincaré equation for $\mathbf{U}$, see Holm, Marsden and Ratiu [1], [2], 


$$
\left(\frac{\partial}{\partial t}+\mathbf{U} \cdot \nabla\right) \frac{1}{D} \frac{\delta L}{\delta \mathbf{U}}+\frac{1}{D} \frac{\delta L}{\delta U^{j}} \nabla U^{j}-\nabla \frac{\delta L}{\delta D}=0
$$

(throughout, we sum on repeated indices) and the Lagrange multiplier $P$ imposes the constraint,

$$
\frac{\delta L}{\delta P}=0
$$

Substituting the variational derivatives of the Lagrangian (2.10),

$$
\frac{1}{D} \frac{\delta L}{\delta \mathbf{U}}=\mathbf{U}, \quad \frac{\delta L}{\delta D}=\frac{1}{2}|\mathbf{U}|^{2}-P, \quad \frac{\delta L}{\delta P}=1-D,
$$

into the Euler-Poincaré equation (2.11) with constraint (2.12) and using continuity (2.7) yields Euler's equations for the incompressible motion of an ideal fluid in the Eulerian description, namely,

$$
\left(\frac{\partial}{\partial t}+\mathbf{U} \cdot \boldsymbol{\nabla}\right) \mathbf{U}+\nabla P=0, \quad \boldsymbol{\nabla} \cdot \mathbf{U}=0 .
$$

For Lagrangians of the type (2.1) - specifically those Lagrangians that are invariant under "particle relabeling" (by the right-action of the volume preserving diffeomorphism group acting on the tangent space of the fluid parcel trajectories) - the Euler-Poincaré equations (2.11) are equivalent to the Euler-Lagrange equations (2.3). See Holm, Marsden and Ratiu [1], [2] for more details, discussions and proofs of this type of equivalence.

Of course, the Euler equations (2.14) could also be found by directly transforming the Euler-Lagrange equations (2.4) from the Lagrangian, to the Eulerian description by using the basic relations (2.6). This would avoid the step of transforming Hamilton's principle into the Eulerian description. And this is the point: the equivalence of the Euler-Lagrange equations (2.3) and the Euler-Poincaré equations (2.11) facilitates easy, immediate transitions from one description to the other that are helpful in developing approximate fluid models and interpreting their solution properties. In this paper, we shall take both the Lagrangian, and the Eulerian viewpoints, switching from one to the other whenever it facilitates our purpose in the development of these models. In the end, we shall be writing Eulerian representations of approximate fluid models, found by taking either Lagrangian, or Eulerian means of Hamilton's principle and then applying the Euler-Poincaré theory.

\section{$3 \quad$ Averaged Lagrangians and Euler-Lagrange equations}

\subsection{Lagrangian fluid trajectory fluctuations}

The trajectory of a Lagrangian fluid parcel $\mathbf{X}^{\xi}(\mathbf{a}, t ; \omega)$ may be decomposed into its mean and fluctuating parts as

$$
\mathbf{X}^{\xi}(\mathbf{a}, t ; \omega)=\mathbf{X}(\mathbf{a}, t)+\boldsymbol{\xi}(\mathbf{X}(\mathbf{a}, t), t ; \omega) .
$$


This is the Reynolds decomposition of a Lagrangian fluid trajectory. Here $\boldsymbol{\xi}=$ $\mathbf{X}^{\xi}-\mathbf{X}$ is a rapid (or perhaps random) vector field of fluctuations defined along the Lagrangian fluid trajectory. The independent variable $\omega$ in equation (3.1) denotes either rapid time variation, or random fluctuations. So the variable $\omega$ is allowed a dual interpretation. In its first interpretation, $\omega$ is regarded as a short time scale associated with rapid fluctuations. In its second interpretation, $\omega$ may be regarded as a random variable associated with a stochastic process obeyed by the fluctuations and defined along the Lagrangian fluid trajectory $\mathbf{X}$ with label $\mathbf{a}$ and slow time variation $t$. This variability may be regarded equally well as being either intrinsic, or extrinsic. We may assume the the fluid trajectory is fluctuating intrinsically; or, we may assume the fluid is subjected to a random forcing, which introduces a stochastic component into its acceleration, resulting eventually in the decomposition (3.1) of the Lagrangian fluid trajectory.

We denote by $\langle\cdot\rangle$ the averaging procedure performed at constant Lagrangian label a and time $t$. This averaging is performed either over the rapid time $\omega$,

$$
\langle f\rangle \equiv \lim _{T \rightarrow \infty} \frac{1}{T} \int_{-T}^{T} f(\omega) d \omega,
$$

or, alternatively, with respect to a certain probability distribution $\mathcal{P}(\omega)$ associated with the random event $\omega$,

$$
\langle f\rangle \equiv \int f(\omega) \mathcal{P}(\omega) d \omega, \quad \text { with } \quad \int \mathcal{P}(\omega) d \omega=1
$$

Such an average taken while holding Lagrangian labels a fixed is called a $\mathbf{L a}$ grangian mean. The fluctuations are assumed to have zero Lagrangian mean, $\langle\boldsymbol{\xi}\rangle=0$; so the quantity $\mathbf{X}(\mathbf{a}, t)$ in equation (3.1) is the mean Lagrangian fluid trajectory, since $\left\langle\mathbf{X}^{\xi}(\mathbf{a}, t ; \omega)\right\rangle=\mathbf{X}(\mathbf{a}, t)$. Thus, the quantity $\boldsymbol{\xi}$ describes the fluctuating displacement of the fluid trajectory, relative to its Lagrangian mean. In the presence of these rapid (or random) fluctuations, the Lagrangian $L$ in equation (2.1) appearing in Hamilton's principle (2.2) contains both slowly varying mean variables and rapidly fluctuating, or random variables. Thus, the Lagrangian (2.1) becomes

$$
L(\omega)=\int d^{3} a\left\{\frac{1}{2}\left|\dot{\mathbf{X}}^{\xi}(\mathbf{a}, t ; \omega)\right|^{2}+P\left(\mathbf{X}^{\xi}(\mathbf{a}, t ; \omega), t\right)\left[\operatorname{det}\left(\mathbf{X}_{a}^{\xi \prime}\right)(\mathbf{a}, t ; \omega)-1\right]\right\},
$$

where superscript $\xi$ denotes the Reynolds decomposed Lagrangian fluid trajectory in equation (3.1).

\subsection{Induced Eulerian velocity fluctuations}

The decomposition $\mathbf{X}^{\xi}$ in (3.1) of the Lagrangian fluid trajectory into its mean and fluctuating components implies a corresponding decomposition of the associated Eulerian velocity field. Via the basic kinematic relations for the fluid velocity,

$$
\mathbf{U}(\mathbf{x}+\boldsymbol{\xi}(\mathbf{x}, t ; \omega), t)=\dot{\mathbf{X}}^{\xi}(\mathbf{a}, t ; \omega) \quad \text { for } \quad \mathbf{x}+\boldsymbol{\xi}(\mathbf{x}, t ; \omega)=\mathbf{X}^{\xi}(\mathbf{a}, t ; \omega)
$$


we have

$$
\begin{aligned}
\mathbf{U}(\mathbf{x}+\boldsymbol{\xi}(\mathbf{x}, t ; \omega), t)=\frac{d}{d t}(\mathbf{x}+\boldsymbol{\xi}(\mathbf{x}, t ; \omega)) & =\mathbf{u}(\mathbf{x}, t)+\frac{d}{d t} \boldsymbol{\xi}(\mathbf{x}, t ; \omega), \\
\text { where } \frac{d}{d t} & \equiv \frac{\partial}{\partial t}+\mathbf{u}(\mathbf{x}, t) \cdot \nabla
\end{aligned}
$$

defines the advective time derivative. Since the displacement fluctuations of the fluid trajectory have zero Lagrangian mean, $\langle\boldsymbol{\xi}\rangle=0$, the corresponding velocity fluctuations will also have zero Lagrangian mean, so that $\langle d \boldsymbol{\xi} / d t\rangle=0$, as well. Therefore, upon taking the Lagrangian mean of equation (3.6) we find the relation

$$
\mathbf{u}(\mathbf{x}, t)=\langle\mathbf{U}(\mathbf{x}+\boldsymbol{\xi}, t)\rangle=\dot{\mathbf{X}}(\mathbf{a}, t) .
$$

Thus, the quantity $\mathbf{u}(\mathbf{x}, t)$ appearing equations in (3.6) and (3.7) is the Lagrangian mean fluid velocity at the position $\mathbf{x}=\mathbf{X}(\mathbf{a}, t)$ along the fluid trajectory with Lagrangian label a.

We use the basic relations in (3.5) to transform variables in the Lagrangian (3.4) from labels $\mathbf{a}$, to position along the mean fluid trajectory $\mathbf{x}=\mathbf{X}(\mathbf{a}, t)$, yielding

$$
\begin{aligned}
L(\omega)=\int D d^{3} x\{ & \frac{1}{2}|\mathbf{U}(\mathbf{x}+\boldsymbol{\xi}(\mathbf{x}, t ; \omega), t)|^{2} \\
& \left.+P(\mathbf{x}+\boldsymbol{\xi}(\mathbf{x}, t ; \omega), t)\left[\operatorname{det}\left(\mathbf{X}_{X}^{\xi \prime}\right) \operatorname{det}\left(\mathbf{X}_{a}^{\prime}\right)-1\right]\right\},
\end{aligned}
$$

where the volume element $D$ satisfies $\operatorname{det}\left(\mathbf{X}_{a}^{\prime}\right)=D^{-1}(\mathbf{x}, t)$. Consequently, the time dependence of the product of determinants splits into two factors,

$$
\operatorname{det}\left(\mathbf{X}_{X}^{\xi \prime}\right) \operatorname{det}\left(\mathbf{X}_{a}^{\prime}\right)=\underbrace{D^{-1}(\mathbf{x}, t)}_{\text {slow }} \underbrace{\operatorname{det}(I+\nabla \boldsymbol{\xi}(\mathbf{x}, t ; \omega))}_{\text {fast }(\text { or random })}
$$

Hence we may rewrite equation (3.9) for the Lagrangian $L(\omega)$ in the Eulerian description equivalently as

$$
L(\omega)=\int d^{3} x\left\{\frac{D}{2}|\mathbf{U}(\mathbf{x}+\boldsymbol{\xi}(\mathbf{x}, t ; \omega), t)|^{2}+P(\mathbf{x}+\boldsymbol{\xi}(\mathbf{x}, t ; \omega), t)[\operatorname{det}(I+\nabla \boldsymbol{\xi})-D]\right\}
$$

\subsection{A Taylor series approximation to order $O\left(|\boldsymbol{\xi}|^{2}\right)$}

Until this point in the development, we have made no approximations. Now, though, we shall assume that the magnitude $|\boldsymbol{\xi}|$ of the rapid fluctuations is small enough to allow meaningful Taylor expansions of the unapproximated Eulerian velocity $\mathbf{U}$ and pressure $P$ as,

$$
\begin{aligned}
\mathbf{U}(\mathbf{x}+\boldsymbol{\xi}(\mathbf{x}, t ; \omega), t) & =\mathbf{u}(\mathbf{x}, t)+\frac{d \boldsymbol{\xi}}{d t} \\
& =\mathbf{U}(\mathbf{x}, t)+\boldsymbol{\xi}(\mathbf{x}, t ; \omega) \cdot \nabla \mathbf{U}(\mathbf{x}, t)+O\left(|\boldsymbol{\xi}|^{2}\right) .
\end{aligned}
$$


Taking averages and setting $\langle\boldsymbol{\xi}\rangle=0$ and $\langle d \boldsymbol{\xi} / d t\rangle=0$ in the two expressions for $\mathbf{U}(\mathbf{x}+\boldsymbol{\xi}, t)$ in $(3.12)$ and $(3.13)$ gives

$$
\langle\mathbf{U}(\mathbf{x}+\boldsymbol{\xi}(\mathbf{x}, t ; \omega), t)\rangle \equiv \mathbf{u}(\mathbf{x}, t)=\mathbf{U}(\mathbf{x}, t)+O\left(|\boldsymbol{\xi}|^{2}\right),
$$

where $\mathbf{u}(\mathbf{x}, t)$ is again the Lagrangian mean velocity. Thus, the slowly varying velocities $\mathbf{u}(\mathbf{x}, t)$ and $\mathbf{U}(\mathbf{x}, t)$ differ only at order $O\left(|\boldsymbol{\xi}|^{2}\right)$.

A similar Taylor expansion result holds for pressure,

$$
P(\mathbf{x}+\boldsymbol{\xi}(\mathbf{x}, t ; \omega), t)=P(\mathbf{x}, t)+\boldsymbol{\xi}(\mathbf{x}, t ; \omega) \cdot \nabla P(\mathbf{x}, t)+O\left(|\boldsymbol{\xi}|^{2}\right)
$$

Taking averages then gives a relation for the Lagrangian mean pressure, $p$,

$$
\langle P(\mathbf{x}+\boldsymbol{\xi}(\mathbf{x}, t ; \omega), t)\rangle \equiv p(\mathbf{x}, t)=P(\mathbf{x}, t)+O\left(|\boldsymbol{\xi}|^{2}\right) .
$$

Hence, the pressures $p(\mathbf{x}, t)$ and $P(\mathbf{x}, t)$ also differ at order $O\left(|\boldsymbol{\xi}|^{2}\right)$. We shall discuss the physical meaning of equations (3.14) and (3.16) further and model their order $O\left(|\boldsymbol{\xi}|^{2}\right)$ terms in detail later, in Section 4 . Here, we only emphasize that the present approach ascribes all of the rapid variation in velocity $\mathbf{U}(\mathbf{x}+\boldsymbol{\xi}, t)$ and pressure $P(\mathbf{x}+\boldsymbol{\xi}, t)$ to the Lagrangian trajectory fluctuation displacement $\boldsymbol{\xi}(\mathbf{x}, t ; \omega)$; whereas there could be an additional Eulerian fluctuation in the functions $\mathbf{U}$ and $P$, themselves, e.g., one could take $\mathbf{U}(\mathbf{x}+\boldsymbol{\xi}, t ; \omega)$. We shall return to the issue of properly representing the velocity and pressure fluctuations in Section 4 , when we compare their Lagrangian and Eulerian representations.

Taking the difference between equations (3.12) and (3.13) and using equation (3.14) now yields an equation for the modulational dynamics of $\boldsymbol{\xi}(\mathbf{x}, t ; \omega)$ in slow time, valid to order $O\left(|\boldsymbol{\xi}|^{2}\right)$,

$$
\frac{d \boldsymbol{\xi}}{d t}=\frac{\partial \boldsymbol{\xi}}{\partial t}+\mathbf{u}(\mathbf{x}, t) \cdot \nabla \boldsymbol{\xi}=\boldsymbol{\xi} \cdot \nabla \mathbf{u}(\mathbf{x}, t)
$$

This equation is also equivalent to the vector field commutation relation,

$$
\left[\frac{d}{d t}, \boldsymbol{\xi} \cdot \boldsymbol{\nabla}\right]=\left[\frac{\partial}{\partial t}+\mathbf{u}(\mathbf{x}, t) \cdot \nabla, \boldsymbol{\xi} \cdot \boldsymbol{\nabla}\right]=0
$$

Hence, we find the remarkable relation,

$$
\frac{d}{d t} \boldsymbol{\xi} \cdot \boldsymbol{\nabla} \mathbf{A}(\mathbf{x}, t)=\boldsymbol{\xi} \cdot \nabla \frac{d}{d t} \mathbf{A}(\mathbf{x}, t),
$$

for any vector $\mathbf{A}(\mathbf{x}, t)$, provided relation (3.17) holds. We shall see in a moment that this is an exact relation, provided the fluctuation field $\boldsymbol{\xi}$ in the decomposition (3.1) satisfies $\boldsymbol{\xi}(\mathbf{X}(\mathbf{a}, t), t ; \omega)=\left(\tilde{\boldsymbol{\xi}}(\mathbf{a} ; \omega) \cdot \frac{\partial}{\partial \mathbf{a}}\right) \mathbf{X}(\mathbf{a}, t)$, in which the dependences on fast and slow time variables are separated (factored). 


\subsection{Remarks about advected quantities and Taylor's hypothesis}

We shall show that, geometrically, equation (3.17) means that the slow evolution, or modulation, of the fluctuation vector field $\boldsymbol{\xi}(\mathbf{x}, t ; \omega)$ is invariant under the flow of the Lagrangian mean velocity, $\mathbf{u}(\mathbf{x}, t)$. In other words, this vector field is an advected quantity, "frozen" into the flow of the Lagrangian mean velocity. This situation is analogous to either the vorticity stretching equation for the 3D incompressible ideal Euler equations, or the ratio of magnetic field intensity to mass density in ideal magnetohydrodynamics, [1]. Following the solution attributed to Cauchy [8] for the $3 \mathrm{D}$ vorticity stretching equation, the solution of the advection condition (3.17) may be expressed in components in separated form as

$$
\xi^{i}(\mathbf{X}(\mathbf{a}, t ; \omega), t)=\underbrace{F_{A}^{i}(\mathbf{a}, t)}_{\text {slow }} \underbrace{\tilde{\xi}^{A}(\mathbf{a} ; \omega)}_{\text {fast }}, \quad \text { with } \quad F_{A}^{i}=\left(\mathbf{X}_{a}^{\prime}\right)_{A}^{i}=\frac{\partial X^{i}(\mathbf{a}, t)}{\partial a^{A}} .
$$

To verify this solution, one may substitute it into $\partial \tilde{\xi}^{A}(\mathbf{a} ; \omega) /\left.\partial t\right|_{\mathbf{a}}=0$, where $\tilde{\xi}^{A}=$ $\left(F^{-1}\right)_{i}^{A} \xi^{i}$ and $\left(F^{-1}\right)_{i}^{A}$ is the matrix inverse of $F_{A}^{i}$, so that $\left(F^{-1}\right)_{i}^{A} F_{B}^{i}=\delta_{B}^{A}$ and $F_{A}^{i}\left(F^{-1}\right)_{j}^{A}=\delta_{j}^{i}$. The existence of these matrix inverse relations is guaranteed by preservation of fluid volume,

$$
\operatorname{det}\left(\mathbf{X}_{a}^{\prime}\right)=\operatorname{det}\left(F_{A}^{i}\right) \equiv \operatorname{det} F=1 .
$$

We also note the relation,

$$
\dot{F}_{C}^{k}\left(F^{-1}\right)_{j}^{C}(\mathbf{a}, t)=\frac{\partial u^{k}}{\partial x^{j}}(\mathbf{x}, t), \quad \text { for } \quad \mathbf{x}=\mathbf{X}(\mathbf{a}, t) .
$$

Rearranging equation (3.20) implies

$$
\boldsymbol{\xi} \cdot \boldsymbol{\nabla}=\xi^{i}(\mathbf{x}, t ; \omega) \frac{\partial}{\partial x^{i}}=\tilde{\xi}^{A}(\mathbf{a} ; \omega) \frac{\partial}{\partial a^{A}}=\tilde{\boldsymbol{\xi}} \cdot \frac{\partial}{\partial \mathbf{a}} \quad \text { for } \quad \mathbf{x}=\mathbf{X}(\mathbf{a}, t),
$$

where $\tilde{\boldsymbol{\xi}}(\mathbf{a} ; \omega)$ may be taken as the initial value in slow time of the fluctuation $\boldsymbol{\xi}(\mathbf{x}, t ; \omega)$. Thus, along the Lagrangian mean fluid trajectory the vector field $\boldsymbol{\xi} \cdot \boldsymbol{\nabla}$ with parameter $\omega$ may be expressed solely in terms of the Lagrangian labels. This means $\boldsymbol{\xi} \cdot \boldsymbol{\nabla}$ is an advected quantity, invariant in slow time along the Lagrangian mean fluid trajectory, just as indicated by the commutation relation (3.18). Furthermore, to order $O\left(|\boldsymbol{\xi}|^{2}\right)$ the decomposition (3.1) may be expressed using relation (3.23) as

$$
\mathbf{X}^{\xi}(\mathbf{a}, t ; \omega)=\mathbf{X}+\boldsymbol{\xi}(\mathbf{X}, t ; \omega)=\left(1+\tilde{\xi}^{A}(\mathbf{a} ; \omega) \frac{\partial}{\partial a^{A}}\right) \mathbf{X}(\mathbf{a}, t)
$$

Conversely, we may show that advection of the Lagrangian vector field $\tilde{\boldsymbol{\xi}}(\mathbf{a} ; \omega) \cdot \frac{\partial}{\partial \mathbf{a}}$ implies the advection condition (3.17) for the slow time evolution of the Eulerian vector field $\boldsymbol{\xi} \cdot \boldsymbol{\nabla}$, since (in analogy to the calculation (2.9) for the Lagrangian invariance of the volume element),

$$
\begin{aligned}
0=\left.\frac{\partial}{\partial t}\right|_{\mathbf{a}}\left(\tilde{\boldsymbol{\xi}}(\mathbf{a} ; \omega) \cdot \frac{\partial}{\partial \mathbf{a}}\right) & =\left(\frac{\partial}{\partial t}+£_{\mathbf{u}}\right)(\boldsymbol{\xi} \cdot \boldsymbol{\nabla}) \\
& =\left[\frac{\partial \boldsymbol{\xi}}{\partial t}+\mathbf{u} \cdot \boldsymbol{\nabla} \boldsymbol{\xi}-\boldsymbol{\xi} \cdot \boldsymbol{\nabla} \mathbf{u}\right] \cdot \boldsymbol{\nabla}
\end{aligned}
$$


where $£_{\mathbf{u}}$ denotes Lie derivative with respect to the Lagrangian mean fluid velocity, $\mathbf{u}(\mathbf{x}, t)$. Vanishing of the term in brackets recovers the Eulerian invariance equation (3.17), which we now understand is equivalent to assuming the separated form $\boldsymbol{\xi}(\mathbf{X}(\mathbf{a}, t), t ; \omega)=\left(\tilde{\boldsymbol{\xi}}(\mathbf{a} ; \omega) \cdot \frac{\partial}{\partial \mathbf{a}}\right) \mathbf{X}(\mathbf{a}, t)$ in the decomposition (3.1).

Remark. The advection of the fluctuating vector field $\boldsymbol{\xi} \cdot \boldsymbol{\nabla}$ expressed in relation (3.17), or its equivalent commutator form (3.25) is an extended form of the Taylor hypothesis, i.e., that small rapid fluctuation fields are swept downstream with the mean flow [9], 10]. Relation (3.17) extends the traditional interpretation of Taylor's hypothesis to apply to advection of the rapid fluctuations of a vector quantity by the Lagrangian mean flow. The Taylor hypothesis is usually interpreted [10], [11] as meaning that turbulent fluctuations satisfy $\partial / \partial t=-\bar{U} \partial / \partial x$ for a sufficiently short time interval, where $\bar{U}$ is the (constant) Eulerian mean flow velocity in the $x$ direction. The extended form of Taylor's hypothesis we introduce here asserts that the advection relation (3.17) holds along each mean Lagrangian fluid trajectory and accounts for the vector nature of the displacement fluctuation, $\boldsymbol{\xi}$. According to its derivation in the steps leading to equation (3.17), this extended Taylor's hypothesis is valid to order $O\left(|\boldsymbol{\xi}|^{2}\right)$. When $\mathbf{u}$ is replaced by $(\bar{U}, 0,0)$ with constant $\bar{U}$ the advection relation (3.17) reduces to the traditional form of Taylor's hypothesis, which is usually applied to scalar quantities, 11].

\subsection{Averaged approximate Lagrangians}

After the approximations leading to the Taylor hypothesis relation (3.17) with its geometrical interpretation (3.25), our Lagrangian $L(\omega)$ in (3.11) becomes

$$
\begin{aligned}
L(\omega)= & \int d^{3} x\left\{D \frac{1}{2}|(1+\boldsymbol{\xi}(\mathbf{x}, t ; \omega) \cdot \boldsymbol{\nabla}) \mathbf{u}(\mathbf{x}, t)|^{2}\right. \\
& +[(1+\boldsymbol{\xi}(\mathbf{x}, t ; \omega) \cdot \boldsymbol{\nabla}) p(\mathbf{x}, t)][\operatorname{det}(I+\boldsymbol{\nabla} \boldsymbol{\xi})-D(\mathbf{x}, t)]\},
\end{aligned}
$$

in which $p$ is the Lagrangian mean pressure. Using relation (3.23) transforms this formula to the Lagrangian picture as, cf. equations (2.1) and (3.4),

$$
\begin{aligned}
L(\omega)= & \int d^{3} a\left\{\frac{1}{2}\left|\left(1+\tilde{\xi}^{A}(\mathbf{a} ; \omega) \frac{\partial}{\partial a^{A}}\right) \dot{\mathbf{X}}(\mathbf{a}, t)\right|^{2}\right. \\
& \left.+\left[\left(1+\tilde{\xi}^{A}(\mathbf{a} ; \omega) \frac{\partial}{\partial a^{A}}\right) p(\mathbf{X}(\mathbf{a}, t), t)\right]\left[\operatorname{det}\left(\mathbf{X}_{a}^{\xi \prime}\right)(\mathbf{a}, t ; \omega)-1\right]\right\} .
\end{aligned}
$$

Therefore, we may take the Lagrangian mean of this approximate form of $L(\omega)$ to find the following averaged approximate Lagrangian (using $\langle\tilde{\boldsymbol{\xi}}\rangle=0$ )

$$
\begin{aligned}
\langle L\rangle=\int & d^{3} a\left\{\frac{1}{2}\left[|\dot{\mathbf{X}}(\mathbf{a}, t)|^{2}+\left\langle\tilde{\xi}^{A} \tilde{\xi}^{B}\right\rangle\left(\frac{\partial \dot{\mathbf{X}}}{\partial a^{A}} \cdot \frac{\partial \dot{\mathbf{X}}}{\partial a^{B}}\right)\right]\right. \\
& +p(\mathbf{X}(\mathbf{a}, t), t)\left[\left\langle\operatorname{det}\left(I+\frac{\boldsymbol{\partial} \boldsymbol{\xi}}{\boldsymbol{\partial X}}\right)\right\rangle \operatorname{det}\left(\mathbf{X}_{a}^{\prime}\right)-1\right] \\
& \left.+\operatorname{det}\left(\mathbf{X}_{a}^{\prime}\right)\left(\left\langle\boldsymbol{\xi} \operatorname{det}\left(I+\frac{\boldsymbol{\partial} \boldsymbol{\xi}}{\boldsymbol{\partial X}}\right)\right\rangle \cdot \frac{\boldsymbol{\partial}}{\boldsymbol{\partial X}}\right) p(\mathbf{X}(\mathbf{a}, t), t)\right\} .
\end{aligned}
$$


In the Eulerian description, this averaged approximate Lagrangian becomes

$$
\begin{aligned}
\langle L\rangle= & \int d^{3} x\left\{\frac{D}{2}\left[|\mathbf{u}|^{2}+\left\langle\xi^{k} \xi^{l}\right\rangle\left(\mathbf{u}_{, k} \cdot \mathbf{u}_{, l}\right)\right]\right. \\
& +p[\langle\operatorname{det}(I+\nabla \boldsymbol{\xi})\rangle-D]+(\langle\boldsymbol{\xi} \operatorname{det}(I+\nabla \boldsymbol{\xi})\rangle \cdot \nabla) p\},
\end{aligned}
$$

where partial spatial derivatives are denoted by subscript comma, e.g., $\mathbf{u}_{, k}=\partial \mathbf{u} / \partial x^{k}=$ $\partial_{k} \mathbf{u}$, for $k=1,2,3$. In preparation for using the Euler-Poincaré theorem, we compute the following the following variational derivatives of the averaged approximate Lagrangian,

$$
\begin{aligned}
\frac{1}{D} \frac{\delta\langle L\rangle}{\delta \mathbf{u}} & =\mathbf{u}-\frac{1}{D}\left(\partial_{k} D\left\langle\xi^{k} \xi^{l}\right\rangle \partial_{l}\right) \mathbf{u}, \\
\frac{\delta\langle L\rangle}{\delta D} & =-p+\frac{1}{2}|\mathbf{u}|^{2}+\frac{1}{2}\left\langle\xi^{k} \xi^{l}\right\rangle\left(\mathbf{u}_{, k} \cdot \mathbf{u}_{, l}\right) \equiv-P_{t o t}, \\
\frac{\delta\langle L\rangle}{\delta p} & =\langle\operatorname{det}(I+\nabla \boldsymbol{\xi})\rangle-D-\nabla \cdot\langle\boldsymbol{\xi} \operatorname{det}(I+\nabla \boldsymbol{\xi})\rangle .
\end{aligned}
$$

The natural boundary condition $\mathbf{u} \cdot \hat{\mathbf{n}}=0$ at a fixed boundary with unit normal vector $\hat{\mathbf{n}}$ is imposed on the Lagrangian mean velocity in the course of deriving the Euler-Poincaré equations. In addition, when taking the variations in (3.30), we assume in integrating by parts that the fluctuations do not penetrate a fixed boundary, so that $\boldsymbol{\xi} \cdot \hat{\mathbf{n}}=0$ must be satisfied at such a boundary. Consequently, we have $\hat{n}_{k}\left\langle\xi^{k} \xi^{l}\right\rangle=0$ at fixed boundaries, as well. We shall discuss variations with respect to $\left\langle\xi^{k} \xi^{l}\right\rangle$ after making further approximations in $\langle L\rangle$.

\subsection{Further approximations}

Stationarity of the averaged approximate Lagrangian $\langle L\rangle$ in (3.29) and the last equation in (3.30) imply

$$
D=\langle\operatorname{det}(I+\nabla \boldsymbol{\xi})\rangle-\nabla \cdot\langle\boldsymbol{\xi} \operatorname{det}(I+\nabla \boldsymbol{\xi})\rangle .
$$

We shall approximate this relation using the identity,

$$
\langle\operatorname{det}(I+\nabla \boldsymbol{\xi})\rangle=1+\nabla \cdot\langle\boldsymbol{\xi}\rangle+\frac{1}{2} \boldsymbol{\nabla} \cdot\langle\boldsymbol{\xi} \boldsymbol{\nabla} \cdot \boldsymbol{\xi}-\boldsymbol{\xi} \cdot \nabla \boldsymbol{\xi}\rangle+\langle\operatorname{det}(\boldsymbol{\nabla} \boldsymbol{\xi})\rangle .
$$

Since we assume $|\boldsymbol{\xi}|$ to be small (at least typically, or in an average sense), the relation (3.31) can be given a simple and concise approximate form by neglecting terms of order $O\left(|\boldsymbol{\xi}|^{3}\right)$, or higher, involving $\langle\operatorname{det}(\boldsymbol{\nabla} \boldsymbol{\xi})\rangle$ and $\boldsymbol{\nabla} \cdot\langle\boldsymbol{\xi} \operatorname{det}(\boldsymbol{\nabla} \boldsymbol{\xi})\rangle$. Namely,

$$
D=1-\frac{1}{2} \boldsymbol{\nabla} \cdot[\langle\boldsymbol{\xi} \cdot \boldsymbol{\nabla} \boldsymbol{\xi}\rangle+\langle\boldsymbol{\xi}(\boldsymbol{\nabla} \cdot \boldsymbol{\xi})\rangle]=1-\frac{1}{2}\left\langle\xi^{k} \xi^{l}\right\rangle_{, k l}
$$

This order $O\left(|\boldsymbol{\xi}|^{2}\right)$ compressibility due to the presence of the fluctuations is consistent with the findings of generalized Lagrangian mean (GLM) theory, [12], [13], [14]. 
Upon substituting the order $O\left(|\boldsymbol{\xi}|^{2}\right)$ approximations yielding relation (3.33) into the Lagrangian (3.28), we find the following averaged approximate Lagrangian,

$$
\langle L\rangle=\int d^{3} x\left\{\frac{D}{2}\left[|\mathbf{u}|^{2}+\left\langle\xi^{k} \xi^{l}\right\rangle\left(\mathbf{u}_{, k} \cdot \mathbf{u}_{, l}\right)\right]+p\left[1-D-\frac{1}{2}\left\langle\xi^{k} \xi^{l}\right\rangle_{, k l}\right]\right\} .
$$

This approximate $\langle L\rangle$ returns to the Lagrangian picture as, cf. equations (2.1), (3.4) and (3.28),

$$
\begin{aligned}
\langle L\rangle= & \int d^{3} a\left\{\frac{1}{2}\left[|\dot{\mathbf{X}}(\mathbf{a}, t)|^{2}+\left\langle\tilde{\xi}^{A} \tilde{\xi}^{B}\right\rangle\left(\frac{\partial \dot{\mathbf{X}}}{\partial a^{A}} \cdot \frac{\partial \dot{\mathbf{X}}}{\partial a^{B}}\right)\right]\right. \\
& +p(\mathbf{X}(\mathbf{a}, t), t)\left[\operatorname{det}\left(\mathbf{X}_{a}^{\prime}\right)-1\right] \\
& \left.-\frac{1}{2} \operatorname{det}\left(\mathbf{X}_{a}^{\prime}\right)\left\langle\tilde{\xi}^{A} \tilde{\xi}^{B}\right\rangle\left[\frac{\partial^{2} p}{\partial a^{A} \partial a^{B}}-\frac{\partial^{2} X^{i}}{\partial a^{A} \partial a^{B}}\left(F^{-1}\right)_{i}^{C} \frac{\partial p}{\partial a^{C}}\right]\right\} .
\end{aligned}
$$

The two equivalent forms (3.34) and (3.35) of the averaged approximate Lagrangian possess order $O\left(|\boldsymbol{\xi}|^{2}\right)$ corrections involving higher order derivatives of the velocity, or pressure, that are contracted using the metric provided by the Lagrangian mean covariance of the fluctuations, either in its Eulerian form, $\left\langle\xi^{k} \xi^{l}\right\rangle$, or in its Lagrangian form, $\left\langle\tilde{\xi}^{A} \tilde{\xi}^{B}\right\rangle$. According to relation (3.20), these two representations of the covariance are related by

$$
\left\langle\tilde{\xi}^{A} \tilde{\xi}^{B}\right\rangle(\mathbf{a})=\left\langle\xi^{k} \xi^{l}\right\rangle(\mathbf{X}, t)\left(F^{-1}\right)_{k}^{A}\left(F^{-1}\right)_{l}^{B} .
$$

This relation and the Piola identity,

$$
\frac{\partial}{\partial a^{A}}\left(\operatorname{det} F\left(F^{-1}\right)_{k}^{A}\right)=0
$$

provide the transformation laws required in converting between the two expressions (3.34) and (3.35) for the averaged approximate Lagrangian.

Remark on the covariance determinant. Equation (3.36) for the transformation of the covariance tensor $\langle\tilde{\xi} \tilde{\xi}\rangle$ as it advects along a Lagrangian fluid trajectory also implies the following relation for its determinant,

$$
\operatorname{det}\langle\tilde{\boldsymbol{\xi}} \tilde{\boldsymbol{\xi}}\rangle(\mathbf{a})=\frac{\operatorname{det}\langle\boldsymbol{\xi} \boldsymbol{\xi}\rangle(\mathbf{X}, t)}{(\operatorname{det} F)^{2}} .
$$

Consequently, the product $D^{2} \operatorname{det}\langle\boldsymbol{\xi} \xi\rangle$ is preserved along fluid trajectories,

$$
\frac{d}{d t}\left(D^{2} \operatorname{det}\langle\xi \xi\rangle\right)=0 \text {. }
$$

Therefore, the covariance of the fluctuations cannot vanish in the course of the motion, provided it is initially nonzero. Thus, one may regard the principle axes of the symmetric tensor $\langle\boldsymbol{\xi} \boldsymbol{\xi}\rangle$ as describing an ellipsoid, that is carried along with each 
fluid parcel and represents an additional "internal" degree of freedom for the fluid, associated with the Lagrangian mean covariance of the advecting fluctuations. This covariance ellipsoid may deform and change orientation as it follows the course of the fluid motion, but its volume (the determinant $\operatorname{det}\langle\boldsymbol{\xi} \xi\rangle$ ) cannot either vanish, or become unbounded, as long as the fluid density $D$ is finite.

Eulerian representation of the covariance dynamics. The time derivative of the relation (3.36) and the basic relation (3.22) imply

$$
0=\left.\frac{\partial}{\partial t}\right|_{\mathbf{a}}\left\langle\tilde{\xi}^{A} \tilde{\xi}^{B}\right\rangle(\mathbf{a})=\left(\frac{d}{d t}\left\langle\xi^{k} \xi^{l}\right\rangle-\left\langle\xi^{k} \xi^{j}\right\rangle u_{, j}^{l}-u_{, j}^{k}\left\langle\xi^{j} \xi^{l}\right\rangle\right)\left(F^{-1}\right)_{k}^{A}\left(F^{-1}\right)_{l}^{B} .
$$

Hence, the Eulerian dynamics for the Lagrangian mean covariance $\left\langle\xi^{k} \xi^{l}\right\rangle$ may be expressed as

$$
\frac{d}{d t}\left\langle\xi^{k} \xi^{l}\right\rangle=\left\langle\xi^{k} \xi^{j}\right\rangle u_{, j}^{l}+u_{, j}^{k}\left\langle\xi^{j} \xi^{l}\right\rangle
$$

or, in vector form,

$$
\frac{d}{d t}\langle\xi \xi\rangle=\langle\xi \xi\rangle \cdot \nabla \mathbf{u}+\nabla \mathbf{u}^{\mathrm{T}} \cdot\langle\boldsymbol{\xi} \boldsymbol{\xi}\rangle
$$

\subsection{The Euler-Lagrange equations with order $O\left(|\xi|^{2}\right)$ compressibil- ity}

The Euler-Lagrange equations of the averaged approximate Lagrangian (3.35),

$$
\left.\frac{\partial}{\partial t}\right|_{\mathbf{a}} \frac{\delta\langle L\rangle}{\delta \dot{\mathbf{X}}}-\frac{\delta\langle L\rangle}{\delta \mathbf{X}}=0
$$

are given by (dropping endpoint contributions),

$$
\left(1-\frac{\partial}{\partial a^{A}}\left\langle\tilde{\xi}^{A} \tilde{\xi}^{B}\right\rangle \frac{\partial}{\partial a^{B}}\right) \ddot{\mathbf{X}}-\frac{\delta\langle L\rangle}{\delta \mathbf{X}}=0 .
$$

We shall defer our discussion of $\delta\langle L\rangle / \delta \mathbf{X}$ until later. Stationarity of $\langle L\rangle$ in 3.35 under variations in $p$ implies order $O\left(|\boldsymbol{\xi}|^{2}\right)$ compressibility,

$$
\operatorname{det} F=1+\frac{1}{2}\left(\operatorname{det} F\left\langle\tilde{\xi}^{A} \tilde{\xi}^{B}\right\rangle\right)_{, A B}+\frac{1}{2} \operatorname{det} F\left(F^{-1}\right)_{i}^{C} \frac{\partial}{\partial a^{C}}\left(\left\langle\tilde{\xi}^{A} \tilde{\xi}^{B}\right\rangle X_{, A B}^{i}\right) .
$$

Equivalently, by using equations (3.36) and (3.37) we find the expected relation,

$$
1=D+\frac{1}{2}\left\langle\xi^{k} \xi^{l}\right\rangle_{, k l}
$$

To write the Eulerian form of the motion equation (3.44) we must transform the operator,

$$
\tilde{\Delta} \equiv \frac{\partial}{\partial a^{A}}\left\langle\tilde{\xi}^{A} \tilde{\xi}^{B}\right\rangle \frac{\partial}{\partial a^{B}}
$$


from the Lagrangian, to the Eulerian description. For this, we use the metric transformation formula (3.36), the definition (3.21) and the Piola identity (3.37) to find the transformation law for $\tilde{\Delta}$,

$$
\begin{aligned}
\tilde{\Delta} & \equiv \frac{\partial}{\partial a^{A}}\left\langle\tilde{\xi}^{A} \tilde{\xi}^{B}\right\rangle \frac{\partial}{\partial a^{B}}=\frac{\partial}{\partial a^{A}}\left\langle\xi^{k} \xi^{l}\right\rangle\left(F^{-1}\right)_{k}^{A}\left(F^{-1}\right)_{l}^{B} \frac{\partial}{\partial a^{B}} \\
& =\operatorname{det} F\left(F^{-1}\right)_{k}^{A} \frac{\partial}{\partial a^{A}} \frac{\left\langle\xi^{k} \xi^{l}\right\rangle}{\operatorname{det} F}\left(F^{-1}\right)_{l}^{B} \frac{\partial}{\partial a^{B}} \\
& =\operatorname{det} F \frac{\partial}{\partial x^{k}} \frac{\left\langle\xi^{k} \xi^{l}\right\rangle}{\operatorname{det} F} \frac{\partial}{\partial x^{l}}=D^{-1} \frac{\partial}{\partial x^{k}} D\left\langle\xi^{k} \xi^{l}\right\rangle \frac{\partial}{\partial x^{l}} \equiv \tilde{\Delta}_{D} .
\end{aligned}
$$

Consequently, we also have the remarkable commutation relation between the operators $d / d t$ and $\tilde{\Delta}_{D}$,

$$
\frac{d}{d t}\left(D^{-1} \frac{\partial}{\partial x^{k}} D\left\langle\xi^{k} \xi^{l}\right\rangle \frac{\partial}{\partial x^{l}}\right)-\left(D^{-1} \frac{\partial}{\partial x^{k}} D\left\langle\xi^{k} \xi^{l}\right\rangle \frac{\partial}{\partial x^{l}}\right) \frac{d}{d t} \equiv\left[\frac{d}{d t}, \tilde{\Delta}_{D}\right]=0 .
$$

This relation allows us to transform the acceleration term in equation (3.44) from the Lagrangian, to the Eulerian description, as

$$
\left(\frac{\partial}{\partial t}+\mathbf{u} \cdot \boldsymbol{\nabla}\right)\left(1-\tilde{\Delta}_{D}\right) \mathbf{u}-\frac{\delta\langle L\rangle}{\delta \mathbf{X}}=0
$$

or, equivalently,

$$
\left(1-\tilde{\Delta}_{D}\right)\left(\frac{\partial}{\partial t}+\mathbf{u} \cdot \nabla\right) \mathbf{u}-\frac{\delta\langle L\rangle}{\delta \mathbf{X}}=0 .
$$

In both forms of this motion equation, the divergence of the Lagrangian mean velocity is given by

$$
\boldsymbol{\nabla} \cdot \mathbf{u}=-\frac{1}{D} \frac{d D}{d t}=\frac{1}{2-\left\langle\xi^{k} \xi^{l}\right\rangle_{, k l}} \frac{d}{d t}\left\langle\xi^{k} \xi^{l}\right\rangle_{, k l}=\frac{1}{2} \frac{d}{d t}\left\langle\xi^{k} \xi^{l}\right\rangle_{, k l}+O\left(|\boldsymbol{\xi}|^{4}\right) .
$$

In its first form $(3.50)$ the presence of the operator $\left(1-\tilde{\Delta}_{D}\right)$ in this motion equation smoothes the Lagrangian mean transport velocity $\mathbf{u}$ relative to the momentum, or circulation velocity $\mathbf{v}=\left(1-\tilde{\Delta}_{D}\right) \mathbf{u}$. In its second form (3.51) the operator $\left(1-\tilde{\Delta}_{D}\right)$ acts to smooth the generalized force $\delta\langle L\rangle / \delta \mathbf{X}$ in an adaptive fashion depending on the Lagrangian mean covariance $\langle\boldsymbol{\xi} \boldsymbol{\xi}\rangle$, which in turn depends on the velocity shear $\boldsymbol{\nabla u}$. At the moment, the generalized force $\delta\langle L\rangle / \delta \mathbf{X}$ is still a Lagrangian quantity. We shall see in Section 6 how to complete the expression of these equations by computing $\delta\langle L\rangle / \delta \mathbf{X}$ in purely Eulerian form in terms of a fluctuation stress tensor.

Since the averaged Lagrangian in equation (3.35) has no explicit time dependence, Noether's theorem implies conservation of energy,

$$
\begin{aligned}
E & =\frac{1}{2} \int d^{3} a\left\{|\dot{\mathbf{X}}(\mathbf{a}, t)|^{2}+\left\langle\tilde{\xi}^{A} \tilde{\xi}^{B}\right\rangle \frac{\partial \dot{\mathbf{X}}}{\partial a^{A}} \cdot \frac{\partial \dot{\mathbf{X}}}{\partial a^{B}}\right\} \\
& =\frac{1}{2} \int d^{3} a\left\{|\dot{\mathbf{X}}(\mathbf{a}, t)|^{2}+\left\langle\xi^{k} \xi^{l}\right\rangle(\mathbf{X}, t)\left(F^{-1}\right)_{k}^{A}\left(F^{-1}\right)_{l}^{B} \frac{\partial \dot{\mathbf{X}}}{\partial a^{A}} \cdot \frac{\partial \dot{\mathbf{X}}}{\partial a^{B}}\right\}, \\
& =\frac{1}{2} \int d^{3} x D\left[|\mathbf{u}|^{2}+\left\langle\xi^{k} \xi^{l}\right\rangle\left(\mathbf{u}_{, k} \cdot \mathbf{u}_{, l}\right)\right] \\
& =\frac{1}{2} \int d^{3} x\left(1-\frac{1}{2}\left\langle\xi^{i} \xi^{j}\right\rangle_{, i j}\right)\left[|\mathbf{u}|^{2}+\left\langle\xi^{k} \xi^{l}\right\rangle\left(\mathbf{u}_{, k} \cdot \mathbf{u}_{, l}\right)\right]
\end{aligned}
$$


where we have used the advection solution (3.36) for the Lagrangian mean covariance in transforming between the first and second lines, and then transformed into the Eulerian description in the last line, cf. equation (3.34). The conserved energy $E$ in (3.53) is the total kinetic energy of the Lagrangian mean motion equation (3.44), including the mean contribution from the covariance of the rapid (or random) fluctuations. We note that $E$ contains a term of order $O\left(|\boldsymbol{\xi}|^{4}\right)$.

\subsection{Restoring incompressibility in the order $O\left(|\xi|^{2}\right)$ model}

We now simplify matters by restoring incompressibility of the Lagrangian mean fluid velocity, thereby producing a simplified averaged approximate Lagrangian, cf. equation (3.34)

$$
\langle L\rangle=\int d^{3} x\left\{\frac{D}{2}\left[|\mathbf{u}|^{2}+\left\langle\xi^{k} \xi^{l}\right\rangle\left(\mathbf{u}_{, k} \cdot \mathbf{u}_{, l}\right)\right]+p[1-D]\right\} .
$$

Here, to regain some simplicity in the analysis below, we dropped terms in the pressure constraint (3.33) of order $O\left(|\boldsymbol{\xi}|^{2}\right)$, but kept terms of the same order in the kinetic energy piece of the mean Lagrangian in (3.29). The approximate $\langle L\rangle$ in (3.54) so obtained returns to the Lagrangian picture as, cf. equations (2.1), (3.4), (3.28) and (3.35),

$$
\begin{aligned}
\langle L\rangle=\int d^{3} a\left\{\frac { 1 } { 2 } \left[|\dot{\mathbf{X}}(\mathbf{a}, t)|^{2}\right.\right. & \left.+\left\langle\tilde{\xi}^{A} \tilde{\xi}^{B}\right\rangle\left(\frac{\partial \dot{\mathbf{X}}}{\partial a^{A}} \cdot \frac{\partial \dot{\mathbf{X}}}{\partial a^{B}}\right)\right] \\
& \left.+p(\mathbf{X}(\mathbf{a}, t), t)\left[\operatorname{det}\left(\mathbf{X}_{a}^{\prime}\right)-1\right]\right\}
\end{aligned}
$$

The particular averaged approximate Lagrangian $\langle L\rangle$ in (3.35) also could have been obtained by substituting the approximate decomposition in equation (3.24) directly into the kinetic energy term of the original Lagrangian (2.1) and then averaging.

Relative to the starting Lagrangian (2.1) for Euler's incompressible fluid equations, our procedure of decomposition, approximation and averaging, followed by restoring incompressibility of $\mathbf{u}$ has merely introduced an additional term into the kinetic energy of the averaged approximate Lagrangian $\langle L\rangle$ in (3.35). This additional term involves higher order derivatives of the velocity, that are contracted using the metric defined by the pullback of the advected Lagrangian mean covariance of the fluctuations, namely, $\left\langle\tilde{\xi}^{A} \tilde{\xi}^{B}\right\rangle$, as given in equation (3.36).

\subsection{The Euler-Lagrange equations for the LMM model}

The Euler-Lagrange equations (2.3) for the simplified averaged approximate Lagrangian (3.55) are immediately obtained as

$$
\left(1-\frac{\partial}{\partial a^{A}}\left\langle\tilde{\xi}^{A} \tilde{\xi}^{B}\right\rangle \frac{\partial}{\partial a^{B}}\right) \ddot{\mathbf{X}}+\operatorname{det}\left(\mathbf{X}_{a}^{\prime}\right) \nabla p=0
$$


and stationarity under variations in $p$ implies volume preservation, $\operatorname{det}\left(\mathbf{X}_{a}^{\prime}\right)=1$. To write the Eulerian form of this equation we must transform the operator $\tilde{\Delta}$ as done in equation (3.48). When volume preservation is imposed, this gives

$$
\tilde{\Delta}=\frac{\partial}{\partial a^{A}}\left\langle\tilde{\xi}^{A} \tilde{\xi}^{B}\right\rangle \frac{\partial}{\partial a^{B}}=\frac{\partial}{\partial x^{k}}\left\langle\xi^{k} \xi^{l}\right\rangle \frac{\partial}{\partial x^{l}}=\left.\tilde{\Delta}_{D}\right|_{D=1} \quad \text { for } \quad D=1 .
$$

This relation allows us to transform equation (3.44) from the Lagrangian, to the Eulerian description, as

$$
\left(\frac{\partial}{\partial t}+\mathbf{u} \cdot \boldsymbol{\nabla}\right)(1-\tilde{\Delta}) \mathbf{u}+\nabla p=0, \quad \text { with } \quad \nabla \cdot \mathbf{u}=0
$$

or, equivalently,

$$
(1-\tilde{\Delta})\left(\frac{\partial}{\partial t}+\mathbf{u} \cdot \boldsymbol{\nabla}\right) \mathbf{u}+\boldsymbol{\nabla} p=0, \quad \text { with } \quad \boldsymbol{\nabla} \cdot \mathbf{u}=0 .
$$

These are two equivalent forms of the Lagrangian mean motion (LMM) equation. In its first form, the presence of the Helmholtz operator $(1-\tilde{\Delta})$ in this motion equation smoothes the Lagrangian mean transport velocity $\mathbf{u}$ relative to the momentum, or circulation velocity $\mathbf{v}=(1-\tilde{\Delta}) \mathbf{u}$. In its second form, the Helmholtz operator $(1-\tilde{\Delta})$ acts to smooth the pressure gradient in an adaptive fashion depending on the covariance $\langle\boldsymbol{\xi} \boldsymbol{\xi}\rangle$, which in turn depends on the velocity shear, $\boldsymbol{\nabla u}$.

Since the averaged Lagrangian in equation (3.55) has no explicit time dependence, Noether's theorem implies conservation of energy, cf. equation (3.53)

$$
\begin{aligned}
E & =\frac{1}{2} \int d^{3} a\left\{|\dot{\mathbf{X}}(\mathbf{a}, t)|^{2}+\left\langle\tilde{\xi}^{A} \tilde{\xi}^{B}\right\rangle \frac{\partial \dot{\mathbf{X}}}{\partial a^{A}} \cdot \frac{\partial \dot{\mathbf{X}}}{\partial a^{B}}\right\} \\
& =\frac{1}{2} \int d^{3} a\left\{|\dot{\mathbf{X}}(\mathbf{a}, t)|^{2}+\left\langle\xi^{k} \xi^{l}\right\rangle(\mathbf{X}, t)\left(F^{-1}\right)_{k}^{A}\left(F^{-1}\right)_{l}^{B} \frac{\partial \dot{\mathbf{X}}}{\partial a^{A}} \cdot \frac{\partial \dot{\mathbf{X}}}{\partial a^{B}}\right\} \\
& =\frac{1}{2} \int d^{3} x D\left[|\mathbf{u}|^{2}+\left\langle\xi^{k} \xi^{l}\right\rangle\left(\mathbf{u}_{, k} \cdot \mathbf{u}_{, l}\right)\right]
\end{aligned}
$$

and $D=1$ for the incompressible LMM case.

\section{Physical interpretations of $\mathrm{u}$ and $\mathrm{v}$ as the Lagrangian and Eulerian mean fluid velocities}

In this section we use the extended Taylor hypothesis (3.17) to interpret the velocities $\mathbf{u}$ and $\mathbf{v}$ in the Lagrangian mean motion (LMM) model by comparing the results of Section 3 with the results of the traditional Reynolds fluid velocity decomposition.

The interpretations are as follows: $\mathbf{u}=\langle\mathbf{U}\rangle^{L}$ is defined as the Lagrangian mean fluid velocity (denoted as $\langle\mathbf{U}\rangle^{L}$ in this section only) and $\mathbf{v}=\left(1-\tilde{\Delta}_{D}\right) \mathbf{u}$ is approximately the Eulerian mean fluid velocity $\langle\mathbf{U}\rangle^{E}$. Here, $\tilde{\Delta}_{D}$ defined in equation (3.48) is the Laplacian operator whose metric is given by the Lagrangian mean displacement covariance $\left\langle\xi^{k} \xi^{l}\right\rangle$ for the advected fluctuations. The difference $\mathbf{u}-\mathbf{v} \equiv\langle\mathbf{U}\rangle^{S}$ 
is called the Stokes mean drift velocity. We shall show that $\langle\mathbf{U}\rangle^{S}$ satisfies the closure relation $\langle\mathbf{U}\rangle^{S}=\tilde{\Delta}_{D} \mathbf{u}$. Thus, we shall identify $\mathbf{v}=\mathbf{u}-\tilde{\Delta}_{D} \mathbf{u}$ as the Eulerian mean fluid velocity $\langle\mathbf{U}\rangle^{E}=\langle\mathbf{U}\rangle^{L}-\langle\mathbf{U}\rangle^{S}$.

This section is meant to be self contained. The notation we use here for averaged quantities such as $\langle\mathbf{U}\rangle^{L},\langle\mathbf{U}\rangle^{E}$ and $\langle\mathbf{U}\rangle^{S}$ is intended to be as self-explanatory as possible.

\subsection{Stokes mean drift closure relation}

The Reynolds decomposition of the Lagrangian fluid trajectory $\mathbf{X}^{\xi}$ introduced in equation (3.1) implies relation (3.6) for the Lagrangian mean fluid velocity $\mathbf{u}$, denoted in this section as $\langle\mathbf{U}\rangle^{L}$,

$$
\mathbf{U}(\mathbf{x}+\boldsymbol{\xi}(\mathbf{x}, t ; \omega), t) \equiv\langle\mathbf{U}\rangle^{L}(\mathbf{x}, t)+\mathbf{U}^{\ell}(\mathbf{x}, t ; \omega), \quad \text { with } \quad \mathbf{U}^{\ell} \equiv \frac{d \boldsymbol{\xi}}{d t}(\mathbf{x}, t ; \omega) .
$$

Note that the slow-time advective derivative,

$$
\frac{d}{d t}=\frac{\partial}{\partial t}+\langle\mathbf{U}\rangle^{L}(\mathbf{x}, t) \cdot \nabla
$$

acts only on $(\mathbf{x}, t)$ dependence and does not act on $\omega$ dependence. As before, the angle brackets as in $\langle\mathbf{U}\rangle^{L}$ denote average over dynamical behavior in $\omega$ that is either rapid in time, or random. In comparison, the traditional Reynolds fluid velocity decomposition is expressed at a given position $\mathrm{x}$ in terms of the Eulerian mean fluid velocity, $\langle\mathbf{U}\rangle^{E}$ as

$$
\mathbf{U}(\mathbf{x}, t ; \omega) \equiv\langle\mathbf{U}\rangle^{E}(\mathbf{x}, t)+\mathbf{U}^{\prime}(\mathbf{x}, t ; \omega) .
$$

We note that the rapid $\omega$-dynamics appears in different functional forms on the left hand sides of equations (4.1) and (4.3) in the definitions of $\langle\mathbf{U}\rangle^{L}$ and $\langle\mathbf{U}\rangle^{E}$. The Reynolds fluid velocity decomposition in (4.3) describes fluctuations in velocity without reference to a Lagrangian parcel trajectory. In principle, such a trajectory could be obtained by integrating this decomposition from an initial reference configuration. Rather than developing the analysis relating these velocity formulas from a Lagrangian viewpoint, we shall instead follow [12] in presenting an heuristic argument relating these velocities as Eulerian quantities by using Taylor series approximations and asymptotics in the magnitude $|\boldsymbol{\xi}|$ of the fluctuations.

Taylor expansion of the Reynolds fluid velocity decomposition (4.3) yields

$$
\begin{aligned}
\mathbf{U}(\mathbf{x}+\boldsymbol{\xi}, t ; \omega)= & \mathbf{U}(\mathbf{x}, t ; \omega)+\boldsymbol{\xi} \cdot \boldsymbol{\nabla} \mathbf{U}(\mathbf{x}, t ; \omega)+\frac{1}{2} \xi^{k} \xi^{l} \mathbf{U}_{, k l}(\mathbf{x}, t ; \omega)+O\left(|\boldsymbol{\xi}|^{3}\right) \\
= & \langle\mathbf{U}\rangle^{E}(\mathbf{x}, t)+\boldsymbol{\xi} \cdot \boldsymbol{\nabla}\langle\mathbf{U}\rangle^{E}(\mathbf{x}, t)+\frac{1}{2} \xi^{k} \xi^{l}\langle\mathbf{U}\rangle_{, k l}^{E}(\mathbf{x}, t) \\
& +\mathbf{U}^{\prime}(\mathbf{x}, t ; \omega)+\boldsymbol{\xi} \cdot \boldsymbol{\nabla} \mathbf{U}^{\prime}(\mathbf{x}, t ; \omega)+O\left(|\boldsymbol{\xi}|^{3}\right)
\end{aligned}
$$

Upon assuming we may equate the different functional forms of velocity arising in the two decompositions as $\mathbf{U}(\mathbf{x}+\boldsymbol{\xi}(\mathbf{x}, t ; \omega), t)=\mathbf{U}(\mathbf{x}+\boldsymbol{\xi}, t ; \omega)$, comparison of the 
two formulas (4.1) and (4.4) gives

$$
\begin{array}{rlrl}
O(1): \quad\langle\mathbf{U}\rangle^{L} & =\langle\mathbf{U}\rangle^{E} \\
O(|\boldsymbol{\xi}|): & \frac{d \boldsymbol{\xi}}{d t} & =\boldsymbol{\xi} \cdot \boldsymbol{\nabla}\langle\mathbf{U}\rangle^{E}+\mathbf{U}^{\prime}(\mathbf{x}, t ; \omega) \\
O\left(|\boldsymbol{\xi}|^{2}\right): & \langle\mathbf{U}\rangle^{S} & \equiv\langle\mathbf{U}\rangle^{L}-\langle\mathbf{U}\rangle^{E} \\
& =\left\langle\boldsymbol{\xi} \cdot \nabla \mathbf{U}^{\prime}\right\rangle(\mathbf{x}, t)+\frac{1}{2}\left\langle\xi^{k} \xi^{l}\right\rangle\langle\mathbf{U}\rangle_{, k l}^{E}(\mathbf{x}, t) .
\end{array}
$$

There is some risk in making this order by order comparison of Taylor expansions, because of the possibility of double counting the fluctuations when comparing their Eulerian and Lagrangian representations. However, there are two cases of the order $O(|\boldsymbol{\xi}|)$ relation $(4.6)$ that are unequivocal:

1. All the fluctuation is modeled in the Lagrangian fluid trajectory. (This is the case studied here.) Then $\mathbf{U}^{\prime}=0$ and (4.6) implies $d \boldsymbol{\xi} / d t=\boldsymbol{\xi} \cdot \boldsymbol{\nabla} \mathbf{u}$. This is the Taylor hypothesis in (3.17).

2. All the fluctuation is modeled in the Eulerian fluid velocity. Then $\mathbf{U}^{\ell}=$ $d \boldsymbol{\xi} / d t=0$ and we obtain $\mathbf{U}^{\prime}=-\boldsymbol{\xi} \cdot \boldsymbol{\nabla}\langle\mathbf{U}\rangle^{E}$ from equation (4.6). The relation $d \boldsymbol{\xi} / d t=0$ is the Taylor-like hypothesis introduced in [23]. We shall investigate this situation in Section 12, when we deal with Eulerian mean fluid theories.

For the Stokes mean drift velocity $\langle\mathbf{U}\rangle^{S} \equiv\langle\mathbf{U}\rangle^{L}-\langle\mathbf{U}\rangle^{E}$ in the present situation with purely Lagrangian fluctuations (so that $\mathbf{U}^{\prime}=0$ ) we shall follow 15 and take

$$
\begin{aligned}
\langle\mathbf{U}\rangle^{S}(\mathbf{x}, t)= & \left\langle\boldsymbol{\xi} \cdot \boldsymbol{\nabla} \mathbf{U}^{\ell}\right\rangle \\
= & (\boldsymbol{\nabla} \cdot\langle\boldsymbol{\xi} \boldsymbol{\xi}\rangle \cdot \boldsymbol{\nabla})\langle\mathbf{U}\rangle^{L} \\
& -\langle(\boldsymbol{\nabla} \cdot \boldsymbol{\xi}) \boldsymbol{\xi}\rangle \cdot \boldsymbol{\nabla}\langle\mathbf{U}\rangle^{L}+\frac{1}{2}\left\langle\xi^{k} \xi^{l}\right\rangle\langle\mathbf{U}\rangle_{, k l}^{L}+O\left(|\boldsymbol{\xi}|^{3}\right) \\
= & (\boldsymbol{\nabla} \cdot\langle\boldsymbol{\xi} \boldsymbol{\xi}\rangle \cdot \boldsymbol{\nabla})\langle\mathbf{U}\rangle^{L}+o\left(|\boldsymbol{\xi}|^{2}\right), \quad(\text { Stokes drift }) .
\end{aligned}
$$

We drop the latter two terms in this expansion, by arguing that

$$
\langle\boldsymbol{\nabla} \cdot \boldsymbol{\xi}\rangle=o(|\boldsymbol{\xi}|) \quad \text { and } \quad\langle\mathbf{U}\rangle_{, k l}^{L}=o(1) .
$$

Hence, we arrive at the approximate closure relation for $\langle\mathbf{U}\rangle^{S}$, to order $o\left(|\boldsymbol{\xi}|^{2}\right)$,

$$
\langle\mathbf{U}\rangle^{S}=\nabla \cdot\left(\langle\boldsymbol{\xi} \boldsymbol{\xi}\rangle \cdot \nabla\langle\mathbf{U}\rangle^{L}\right)=\tilde{\Delta}\langle\mathbf{U}\rangle^{L}
$$

where the operator $\tilde{\Delta}$ is defined by $\tilde{\Delta}=(\boldsymbol{\nabla} \cdot\langle\boldsymbol{\xi} \boldsymbol{\xi}\rangle \cdot \boldsymbol{\nabla})=\tilde{\Delta}_{D}+O\left(|\boldsymbol{\xi}|^{4}\right)$, so we need not distinguish between $\tilde{\Delta}$ and $\tilde{\Delta}_{D}$ here. 
Identification of $\mathbf{v}$ as the Eulerian mean velocity. Subject to the assumptions (4.9) and accepting the definition of $\langle\mathbf{U}\rangle^{S}$ as in (4.8), the momentum, or circulation velocity $\mathbf{v}$ may be identified as the Eulerian mean velocity $\langle\mathbf{U}\rangle^{E}$, by following the chain of relations,

$$
\langle\mathbf{U}\rangle^{E}=\langle\mathbf{U}\rangle^{L}-\langle\mathbf{U}\rangle^{S}=\left(1-\tilde{\Delta}_{D}\right)\langle\mathbf{U}\rangle^{L}=\left(1-\tilde{\Delta}_{D}\right) \mathbf{u}=\mathbf{v} .
$$

This establishes the physical interpretation of the quantity $\mathbf{v}$ as the Eulerian mean velocity.

Lagrangian mean, and Eulerian mean pressures. A similar Taylor expansion may be performed to determine the relation between the Lagrangian mean, and the Eulerian mean pressures. Namely,

$$
P(\mathbf{x}+\boldsymbol{\xi})=P(\mathbf{x})+\boldsymbol{\xi} \cdot \nabla P(\mathbf{x})+\frac{1}{2}\left\langle\xi^{k} \xi^{l}\right\rangle P_{, k l}(\mathbf{x})+O\left(|\boldsymbol{\xi}|^{3}\right) .
$$

Hence, we obtain

$$
\langle P\rangle^{E}(\mathbf{x})=\langle P\rangle^{L}(\mathbf{x})-\frac{1}{2}\left\langle\xi^{k} \xi^{l}\right\rangle\langle P\rangle_{, k l}^{L}(\mathbf{x})+O\left(|\boldsymbol{\xi}|^{3}\right),
$$

which relates the Eulerian mean pressure $\langle P\rangle^{E}$ to the Lagrangian mean pressure $\langle P\rangle^{L}$ to order $O\left(|\boldsymbol{\xi}|^{3}\right)$.

Remarks on boundary conditions. With the interpretation given in (4.11), the Eulerian mean velocity $\mathbf{v}=\langle\mathbf{U}\rangle^{E}$ naturally must be tangent to fixed boundaries, so that

$$
\mathbf{v} \cdot \hat{\mathbf{n}}=0, \quad \text { at a fixed boundary. }
$$

The Lagrangian mean velocity $\mathbf{u}=\langle\mathbf{U}\rangle^{L}$ must satisfy boundary conditions that allow determination of $\mathbf{u}$ from $\mathbf{v}$ by inverting the dynamical Helmholtz operator, $1-\tilde{\Delta}$. For this, we shall choose Dirichlet boundary conditions on the Lagrangian mean velocity,

$$
\mathbf{u}=0, \quad \text { at the boundary. }
$$

We note that $\hat{\mathbf{n}} \cdot\langle\boldsymbol{\xi} \boldsymbol{\xi}\rangle=0$ at the boundary, as well, since the fluctuations should not penetrate the boundary. These remarks provide the rational for choosing the boundary conditions (1.9) for the ideal LMM model.

Relation to the Monin-Yaglom formula. An alternative formula for the Stokes mean drift velocity due to Monin and Yaglom [17 introduces the diffusivity tensor $\boldsymbol{\kappa}$, according to

$$
\langle\mathbf{U}\rangle^{S}=\nabla \cdot \kappa .
$$


Consistency of this formula with relation (4.10) allows one to identify the diffusivity tensor $\boldsymbol{\kappa}$ as

$$
\kappa=\langle\xi \xi\rangle \cdot \nabla\langle\mathbf{U}\rangle^{L}
$$

We decompose this tensor into its symmetric and antisymmetric components, as $\boldsymbol{\kappa}=\boldsymbol{\kappa}_{S}+\boldsymbol{\kappa}_{A}$, where,

$$
\begin{aligned}
\boldsymbol{\kappa}_{S} & =\frac{1}{2}\left(\langle\boldsymbol{\xi} \boldsymbol{\xi}\rangle \cdot \boldsymbol{\nabla}\langle\mathbf{U}\rangle^{L}+\left(\boldsymbol{\nabla}\langle\mathbf{U}\rangle^{L}\right)^{\mathrm{T}} \cdot\langle\boldsymbol{\xi} \boldsymbol{\xi}\rangle\right) \\
\boldsymbol{\kappa}_{A} & =\frac{1}{2}\left(\langle\boldsymbol{\xi} \boldsymbol{\xi}\rangle \cdot \boldsymbol{\nabla}\langle\mathbf{U}\rangle^{L}-\left(\boldsymbol{\nabla}\langle\mathbf{U}\rangle^{L}\right)^{\mathrm{T}} \cdot\langle\boldsymbol{\xi} \boldsymbol{\xi}\rangle\right) .
\end{aligned}
$$

We note from equation (3.42) that

$$
\kappa_{S}=\frac{1}{2} \frac{d}{d t}\langle\xi \xi\rangle
$$

and by direct manipulation we find

$$
\boldsymbol{\nabla} \cdot \boldsymbol{\kappa}_{A}=-\frac{1}{2} \operatorname{curl}\left\langle\boldsymbol{\xi} \times(\boldsymbol{\xi} \cdot \boldsymbol{\nabla})\langle\mathbf{U}\rangle^{L}\right\rangle .
$$

Relation (4.20) for $\boldsymbol{\kappa}_{S}$ is precisely the definition of the Taylor diffusivity [18], 19] and relation (4.21) implies that $\boldsymbol{\kappa}_{A}$ does not contribute to the divergence of $\langle\mathbf{U}\rangle^{S}$. Thus, we find

$$
\nabla \cdot\langle\mathbf{U}\rangle^{S}=\nabla \cdot \boldsymbol{\kappa}_{S}=\nabla \cdot\left(\nabla \cdot \frac{1}{2} \frac{d}{d t}\langle\xi \xi\rangle\right)
$$

In combination with the LMM equations, these identifications provide dynamical equations for the diffusivity in the Monin-Yaglom formula which are of potential use in modeling geophysical fluid dynamics 20].

Comparison with the Craik-Leibovich equations [15], [16]. The explicit expression (4.10) for the Stokes mean drift velocity $\langle\mathbf{U}\rangle^{S}=\mathbf{u}-\mathbf{v}$ allows us to write the LMM motion equation (3.58) in the notation of this Section as

$$
\begin{gathered}
\frac{\partial}{\partial t}\langle\mathbf{U}\rangle^{E}+\langle\mathbf{U}\rangle^{E} \cdot \nabla\langle\mathbf{U}\rangle^{E}+\langle\mathbf{U}\rangle^{S} \cdot \nabla\langle\mathbf{U}\rangle^{E}+\nabla\langle P\rangle^{L}=0, \\
\text { with }\langle\mathbf{U}\rangle^{S}=\tilde{\Delta}\langle\mathbf{U}\rangle^{L}, \quad \text { and } \quad \boldsymbol{\nabla} \cdot\langle\mathbf{U}\rangle^{L}=0 .
\end{gathered}
$$

Thus, the Stokes mean drift velocity introduces an additional transport of Eulerian mean velocity into the ideal LMM motion equation (4.23). The corresponding Kelvin-Noether circulation theorem is

$$
\frac{d}{d t} \oint_{\gamma\left(\langle\mathbf{U}\rangle^{L}\right)}\langle\mathbf{U}\rangle^{E} \cdot d \mathbf{x}=\iint_{S\left(\langle\mathbf{U}\rangle^{L}\right)} \nabla\langle U\rangle_{j}^{E} \times \nabla\langle U\rangle_{j}^{S} \cdot d \mathbf{S},
$$

for any surface $S\left(\langle\mathbf{U}\rangle^{L}\right)$ whose boundary is the closed curve $\gamma\left(\langle\mathbf{U}\rangle^{L}\right)$ moving with the Lagrangian mean fluid velocity $\langle\mathbf{U}\rangle^{L}$. Thus, circulation of Eulerian mean velocity 
will be generated according to these equations whenever the gradients of the Stokes drift velocity and Eulerian mean velocity are not colinear. In this circumstance, the Stokes drift velocity generates convective circulation of the Eulerian mean velocity that tends to reduce the gradient of the Lagrangian mean velocity.

The motion equation (4.23) of the LMM model is reminiscent of (but different from) the ideal Craik-Leibovich (CL) equations [15], [16]. In the CL theory, the rapidly oscillating waves at a free surface are assumed to be unaffected by the more slowly changing currents below. The effect of the waves on the Eulerian mean velocity is parameterized in the CL theory by introducing into the Euler equations a "vortex force," expressed in terms of a prescribed Stokes drift velocity $\langle\mathbf{U}\rangle^{S}(\mathbf{x}, t)$. The CL equations are given by,

$$
\begin{aligned}
& \frac{\partial}{\partial t}\langle\mathbf{U}\rangle^{E}+\langle\mathbf{U}\rangle^{E} \cdot \nabla\langle\mathbf{U}\rangle^{E}-\langle\mathbf{U}\rangle^{S} \times \operatorname{curl}\langle\mathbf{U}\rangle^{E}+\nabla \varpi=0, \\
& \nabla \cdot\langle\mathbf{U}\rangle^{E}=0, \quad \text { and } \quad \varpi=P+\frac{1}{2}\left|\langle\mathbf{U}\rangle^{E}+\langle\mathbf{U}\rangle^{S}\right|^{2}-\frac{1}{2}\left|\langle\mathbf{U}\rangle^{E}\right|^{2} .
\end{aligned}
$$

Here $\varpi$ is a modified pressure term that includes the Eulerian mean pressure $P$ as well as the increase of the kinetic energy of the fluid due to the waves. The term $\langle\mathbf{U}\rangle^{S} \times \operatorname{curl}\langle\mathbf{U}\rangle^{E}$ is the "vortex force" of the CL theory of Langmuir circulation. The Eulerian mean fluid velocity $\langle\mathbf{U}\rangle^{E}$ is assumed to be divergenceless and is required to be tangential to fixed boundaries of the domain of flow.

The first difference from the CL equations is that the ideal LMM model has a dynamical equation for the Stokes mean drift velocity, while this is a prescribed function in the Craik-Leibovich model. Also, the Stokes mean drift velocity appears as a transport term $\left(\langle\mathbf{U}\rangle^{S} \cdot \boldsymbol{\nabla}\langle\mathbf{U}\rangle^{E}\right)$ in the LMM motion equation (4.23), while it appears as a "vortex force" $\left(-\langle\mathbf{U}\rangle^{S} \times \operatorname{curl}\langle\mathbf{U}\rangle^{E}\right)$ in the CL model. Both of these are substantial differences between the LMM model and the Craik-Leibovich theory.

\subsection{An order $O\left(|\boldsymbol{\xi}|^{2}\right)$ model with $\operatorname{div}\langle\mathbf{U}\rangle^{E}=0$}

Rather than the divergenceless condition $\boldsymbol{\nabla} \cdot\langle\mathbf{U}\rangle^{L}=0$ appearing in the LMM equations (1.7), the condition $\boldsymbol{\nabla} \cdot\langle\mathbf{U}\rangle^{E}=0$ is implied by the Eulerian mean of the original divergence free condition $\boldsymbol{\nabla} \cdot \mathbf{U}=0$, when combined with the velocity decomposition (4.3) and the assumption that the Eulerian mean velocity fluctuation has zero Eulerian mean, $\left\langle\mathbf{U}^{\prime}\right\rangle^{E}=0$, where $\langle\cdot\rangle^{E}$ denotes average over the rapid dependence at fixed Eulerian position.

If this were so, then preservation of the condition $\boldsymbol{\nabla} \cdot\langle\mathbf{U}\rangle^{E}=0$ would determine the pressure $p$ in the LMM motion equation (4.23) by solving the Poisson equation $-\Delta p=\nabla \cdot\left(\langle\mathbf{U}\rangle^{L} \cdot \boldsymbol{\nabla}\langle\mathbf{U}\rangle^{E}\right)$ with Neumann boundary conditions obtained by taking the normal component of the motion equation (4.23) and using $\langle\mathbf{U}\rangle^{E} \cdot \hat{\mathbf{n}}=0$ at the boundary. This situation is appealing because of the clear physical interpretation of the velocity variables and their boundary conditions. However, it requires $\boldsymbol{\nabla} \cdot\langle\mathbf{U}\rangle^{L}=O\left(|\boldsymbol{\xi}|^{2}\right)$. Hence, to explore this possibility we must begin by restoring the $O\left(|\boldsymbol{\xi}|^{2}\right)$ compressibility induced by the fluctuations and calculated earlier in 
equation (3.33) as

$$
D=1-\frac{1}{2}\left\langle\xi^{k} \xi^{l}\right\rangle_{, k l}
$$

The arrangements in Hamilton's principle needed for the pressure to impose this relation as a dynamically consistent constraint are discussed in Section 3 leading to the Lagrangian in equation (3.34). It would be convenient if these arrangements were also to imply that $\boldsymbol{\nabla} \cdot\langle\mathbf{U}\rangle^{E}=o\left(|\boldsymbol{\xi}|^{2}\right) \approx 0$, i.e., that the Eulerian mean velocity were incompressible to a certain approximation. We shall now investigate this possibility.

In the notation of the present section, the continuity equation for $D$ as in equation (4.27) implies, to order $O\left(|\boldsymbol{\xi}|^{4}\right)$

$$
\boldsymbol{\nabla} \cdot\langle\mathbf{U}\rangle^{L}=\frac{-1}{D} \frac{d D}{d t}=\frac{1}{2} \frac{d}{d t}\left\langle\xi^{k} \xi^{l}\right\rangle_{, k l}=\frac{1}{2} \frac{d}{d t}(\boldsymbol{\nabla} \cdot \boldsymbol{\nabla} \cdot\langle\boldsymbol{\xi} \boldsymbol{\xi}\rangle) .
$$

Combining this relation with equation (4.10) and the divergence of equation (4.11) gives a formula for the divergence of the Eulerian mean velocity,

$$
\begin{aligned}
\boldsymbol{\nabla} \cdot\langle\mathbf{U}\rangle^{E} & =\boldsymbol{\nabla} \cdot\langle\mathbf{U}\rangle^{L}-\nabla \cdot\langle\mathbf{U}\rangle^{S}=\boldsymbol{\nabla} \cdot\langle\mathbf{U}\rangle^{L}-\nabla \cdot\left(\boldsymbol{\nabla} \cdot\langle\boldsymbol{\xi} \boldsymbol{\xi}\rangle \cdot \boldsymbol{\nabla}\langle\mathbf{U}\rangle^{L}\right) \\
& =\frac{1}{2} \frac{d}{d t}(\boldsymbol{\nabla} \cdot \boldsymbol{\nabla} \cdot\langle\boldsymbol{\xi} \boldsymbol{\xi}\rangle)-\frac{1}{2} \boldsymbol{\nabla} \cdot\left[\boldsymbol{\nabla} \cdot\left(\frac{d}{d t}\langle\boldsymbol{\xi} \boldsymbol{\xi}\rangle\right]\right. \\
& =\frac{1}{2}\left[\frac{d}{d t}, \boldsymbol{\nabla} \cdot \boldsymbol{\nabla} \cdot\right]\langle\boldsymbol{\xi} \boldsymbol{\xi}\rangle=\frac{1}{2}\left[\langle\mathbf{U}\rangle^{L} \cdot \boldsymbol{\nabla}, \boldsymbol{\nabla} \cdot \boldsymbol{\nabla} \cdot\right]\langle\boldsymbol{\xi} \boldsymbol{\xi}\rangle \\
& =O\left(\left|\boldsymbol{\nabla} \boldsymbol{\nabla}\langle\mathbf{U}\rangle^{L}\right|\right) O\left(|\boldsymbol{\xi}|^{2}\right) .
\end{aligned}
$$

Thus, $\boldsymbol{\nabla} \cdot\langle\mathbf{U}\rangle^{E}=o\left(|\boldsymbol{\xi}|^{2}\right) \approx 0$, provided $\left|\nabla \nabla\langle\mathbf{U}\rangle^{L}\right|=o(1)$, as we argued earlier in equation (4.9). Since the Lagrangian mean velocity $\langle\mathbf{U}\rangle^{L}$ is smoothed by Helmholtz inversion, this assumption may be plausible in certain flow regimes. If this asumption is made, the approximate equations that result are

$$
\left(\frac{\partial}{\partial t}+\langle\mathbf{U}\rangle^{L} \cdot \nabla\right)\langle\mathbf{U}\rangle^{E}+\nabla p=0, \quad \nabla \cdot\langle\mathbf{U}\rangle^{E} \approx 0
$$

where

$$
\langle\mathbf{U}\rangle^{L}=(1-\tilde{\Delta})^{-1}\langle\mathbf{U}\rangle^{E}, \quad \tilde{\Delta}=(\boldsymbol{\nabla} \cdot\langle\boldsymbol{\xi} \boldsymbol{\xi}\rangle \cdot \boldsymbol{\nabla})
$$

and

$$
\left(\frac{\partial}{\partial t}+\langle\mathbf{U}\rangle^{L} \cdot \boldsymbol{\nabla}\right)\langle\boldsymbol{\xi} \boldsymbol{\xi}\rangle=\langle\boldsymbol{\xi} \boldsymbol{\xi}\rangle \cdot \boldsymbol{\nabla}\langle\mathbf{U}\rangle^{L}+\left(\boldsymbol{\nabla}\langle\mathbf{U}\rangle^{L}\right)^{\mathrm{T}} \cdot\langle\boldsymbol{\xi} \boldsymbol{\xi}\rangle .
$$

The boundary conditions for the system (4.30) - (4.31) are

$$
\langle\mathbf{U}\rangle^{E} \cdot \hat{\boldsymbol{n}}=0, \quad\langle\mathbf{U}\rangle^{L}=0, \quad \text { and }\langle\boldsymbol{\xi} \boldsymbol{\xi}\rangle \cdot \hat{\boldsymbol{n}}=0 \quad \text { on a fixed boundary. }
$$

The energy for this system is,

$$
E=\frac{1}{2} \int d^{3} x\left(1-\frac{1}{2}\left\langle\xi^{k} \xi^{l}\right\rangle_{, k l}\right)\langle\mathbf{U}\rangle^{E} \cdot\langle\mathbf{U}\rangle^{L} .
$$

This energy will be conserved exactly for $\boldsymbol{\nabla} \cdot\langle\mathbf{U}\rangle^{E}$ satisfying equation 4.29 ) without approximation, as we found in the second order model in Section 3. And it will be conserved approximately for $\boldsymbol{\nabla} \cdot\langle\mathbf{U}\rangle^{E}=0$. 
Summary of Lagrangian mean models with order $O\left(|\xi|^{2}\right)$ compressibility. There are two contending theories with order $O\left(|\boldsymbol{\xi}|^{2}\right)$ compressibility: one has exactly divergenceless Eulerian mean velocity $\langle\mathbf{U}\rangle^{E}$ and only approximate energy conservation; and the other has small divergence of $\langle\mathbf{U}\rangle^{E}$ and exact energy conservation. In fact, the conserved energy in the latter case contains a term of order $O\left(|\boldsymbol{\xi}|^{4}\right)$, which of course is higher order than the validity of the equations. The choice between these two theories probably should be made on the basis of their performance in practice. The divergenceless model defined in equations (4.30) (4.33) may have the advantage of being easier to implement numerically than the nondivergent theory, which consists of equations $(4.30)-4.33$ with $\boldsymbol{\nabla} \cdot\langle\mathbf{U}\rangle^{E} \approx 0$ in equation (4.30) replaced by equation (4.29).

Under mild conditions on $\langle\boldsymbol{\xi} \boldsymbol{\xi}\rangle$, the viscous version of the model in equations (4.30) - (4.33) will dissipate the energy (4.34), if the motion equation is modified to introduce viscosity, as

$$
\left(\frac{\partial}{\partial t}+\langle\mathbf{U}\rangle^{L} \cdot \nabla\right)\langle\mathbf{U}\rangle^{E}+\nabla p=\nu \tilde{\Delta}\langle\mathbf{U}\rangle^{E}, \quad \nabla \cdot\langle\mathbf{U}\rangle^{E} \approx 0 .
$$

If needed, an additional dissipative modification of the Lagrangian mean covariance dynamics could also be proposed. Namely, we propose

$$
\begin{aligned}
\left(\frac{\partial}{\partial t}+\langle\mathbf{U}\rangle^{L} \cdot \nabla\right)\langle\boldsymbol{\xi} \boldsymbol{\xi}\rangle= & \langle\boldsymbol{\xi} \boldsymbol{\xi}\rangle \cdot \boldsymbol{\nabla}\langle\mathbf{U}\rangle^{L}+\left(\boldsymbol{\nabla}\langle\mathbf{U}\rangle^{L}\right)^{\mathrm{T}} \cdot\langle\boldsymbol{\xi} \boldsymbol{\xi}\rangle \\
& -\frac{1}{\tau}\left(\langle\boldsymbol{\xi} \boldsymbol{\xi}\rangle-\alpha^{2} \boldsymbol{I}\right)+\lambda \tilde{\Delta}\langle\boldsymbol{\xi} \boldsymbol{\xi}\rangle .
\end{aligned}
$$

In this equation, $\tau$ is a relaxation time, $\alpha$ is a length scale below which the effects of fluctuations on the mean flow should be suppressed and $\lambda$ is a diffusivity that suppresses gradients of $\langle\boldsymbol{\xi} \boldsymbol{\xi}\rangle$. The quantities $\tau, \alpha$ and $\lambda$ may all be taken as constant parameters, with perhaps $\lambda \sim \alpha^{2} / \tau$. For $\tau^{-1} \geq\left|\nabla\langle\mathbf{U}\rangle^{L}\right|$, the additional dissipation terms in equation (4.36) will cause the covariance $\langle\boldsymbol{\xi} \xi\rangle$ to approach the isotropic, homogeneous conditions represented by the VCHE model, which will be discussed briefly in Section 5. At boundaries one may take $\langle\boldsymbol{\xi} \xi\rangle$ to satisfy equation (4.36) with the $\lambda$ term absent.

\section{One point closure equations}

\subsection{Euler-Poincaré equation for the approximate $\langle L\rangle$}

The Euler-Poincaré equation (2.11) can be written for the approximate $\langle L\rangle$ in equation (3.54) for incompressible flow as

$$
\frac{\partial}{\partial t} \mathbf{v}+(\mathbf{u} \cdot \boldsymbol{\nabla}) \mathbf{v}+v_{j} \boldsymbol{\nabla} u^{j}+\nabla P_{t o t}=0, \quad \boldsymbol{\nabla} \cdot \mathbf{u}=0,
$$

where the momentum conjugate to the velocity $\mathbf{u}$ is given by

$$
\left.\mathbf{v} \equiv \frac{1}{D} \frac{\delta\langle L\rangle}{\delta \mathbf{u}}\right|_{D=1}=\mathbf{u}-\left(\partial_{k}\left\langle\xi^{k} \xi^{l}\right\rangle \partial_{l}\right) \mathbf{u}
$$


The total pressure $P_{t o t}$ in equation (5.1) is defined as, cf. equation (3.30),

$$
P_{t o t} \equiv p-\frac{1}{2}|\mathbf{u}|^{2}-\frac{1}{2}\left\langle\xi^{k} \xi^{l}\right\rangle\left(\mathbf{u}_{, k} \cdot \mathbf{u}_{, l}\right) .
$$

In this Section, the fluctuation covariance $\left\langle\xi^{k} \xi^{l}\right\rangle$ is taken as being independently prescribed, and thus is not varied in Hamilton's principle. Equations (5.1) with definitions (5.2) and (5.3) are generalizations of the $n$-dimensional Camassa-Holm $(\mathrm{CH})$ equations derived in [1], [2]. The latter equations are recovered when the isotropy conditions

$$
\left\langle\xi^{k} \xi^{l}\right\rangle=\alpha^{2} \delta^{k l}
$$

hold and, moreover, the statistics are homogeneous, so that $\alpha^{2}$ is constant. As we shall see in Section 12, there is a natural extension of the $\mathrm{CH}$ model for Eulerian mean fluid dynamics, in which $\mathbf{u}$ is the Eulerian mean fluid velocity and $\mathbf{v}$ is its Lagrangian mean counterpart.

Background. The derivation [1], [2], of the $\mathrm{CH}$ equation (5.1) with definitions (5.2) and (5.3), and homogeneous isotropic Eulerian fluctuation statistics satisfying (5.4) with constant $\alpha^{2}$ generalizes a one dimensional integrable nonlinear dispersive shallow water model [21], [22], to the $n$-dimensional situation and provides the interpretation of $\alpha$ as the typical Eulerian mean amplitude of the fluctuations. See [23] for the extension of that derivation to Riemannian manifolds and discussions of alternative boundary conditions for the case of homogeneous statistics. See also [24] for further analysis of the Riemannian case of the $n$-dimensional $\mathrm{CH}$ equations.

Holm, Marsden and Ratiu [1], [2] note that the conditions of isotropy (5.4) and homogeneity (constant $\alpha^{2}$ ) need to be modified near fixed boundaries, due to the physical requirement that $\boldsymbol{\xi} \cdot \hat{\mathbf{n}}=0$ be satisfied, where $\hat{\mathbf{n}}$ is the unit vector normal to the boundary. However, the condition

$$
\hat{n}_{k}\left\langle\xi^{k} \xi^{l}\right\rangle=0 \text { on the boundary, }
$$

implied by this physical requirement, cannot be satisfied for constant $\alpha^{2}$ in equation (5.4). Chen et al. [3] - [5], overcame this difficulty by allowing spatial variation of $\alpha^{2}$ near fixed boundaries in straight pipe and channel geometries.

\subsection{Relation to one point turbulence closure models}

We note that the velocity $\mathbf{v}$ defined in equation (5.2) is the momentum conjugate to the velocity $\mathbf{u}$ (or dual to $\mathbf{u}$, in the sense of variational derivative of the kinetic energy). On that basis, Chen et al. [3] - [5], proposed the following viscous variant of (5.1), in which viscosity acts to diffuse the momentum $\mathbf{v}$

$$
\frac{\partial}{\partial t} \mathbf{v}+(\mathbf{u} \cdot \boldsymbol{\nabla}) \mathbf{v}+v_{j} \boldsymbol{\nabla} u^{j}=\nu \Delta \mathbf{v}-\nabla P_{t o t}, \quad \boldsymbol{\nabla} \cdot \mathbf{u}=0
$$


In their case, Chen et al. defined the momentum $\mathbf{v}$ and the modified pressure $P$ by

$$
\mathbf{v}=\mathbf{u}-(\boldsymbol{\nabla} \cdot\langle\boldsymbol{\xi}\rangle) \mathbf{u}-\left(\partial_{k} \alpha^{2} \partial_{k}\right) \mathbf{u}
$$

and

$$
P_{t o t} \equiv p-\frac{1}{2}|\mathbf{u}|^{2}-\frac{\alpha^{2}}{2}\left(\mathbf{u}_{, k} \cdot \mathbf{u}_{, k}\right)
$$

Chen et al. [3]- [5], allowed for spatial variation of $\alpha^{2}$, particularly in flow regions near boundaries. They also allowed for the mean $\langle\boldsymbol{\xi}\rangle$ to be nonzero near boundaries to account for the anisotropy there. Chen et al. referred to equation (5.6) with definition (5.7) as the viscous Camassa-Holm equations (VCHE), although this model is also known as the Navier-Stokes alpha model because it reduces to the NavierStokes equations when alpha is absent. They proposed a one point closure model for turbulent flows in pipes and channels by comparing equation (5.6) with the Reynolds averaged Navier-Stokes equations in those geometries and identifying corresponding terms. They then verified the predictions of this closure model by comparison with experimental data at high Reynolds numbers.

Chen et al. [3] - [5], also gave a continuum mechanical interpretation to their VCHE or NS- $\alpha$ closure model, by rewriting (5.6) (in the case where the isotropy conditions (5.4) hold, with $\alpha^{2} \equiv$ constant) in the equivalent constitutive form,

$$
\frac{d \mathbf{u}}{d t}=\operatorname{div} \boldsymbol{T}, \boldsymbol{T}=-p \boldsymbol{I}+2 \nu\left(1-\alpha^{2} \Delta\right) \boldsymbol{D}+2 \alpha^{2} \dot{\boldsymbol{D}},
$$

with $\boldsymbol{\nabla} \cdot \mathbf{u}=0, \boldsymbol{D}=(1 / 2)\left(\boldsymbol{\nabla} \mathbf{u}+\boldsymbol{\nabla} \mathbf{u}^{T}\right), \boldsymbol{\Omega}=(1 / 2)\left(\boldsymbol{\nabla} \mathbf{u}-\boldsymbol{\nabla} \mathbf{u}^{T}\right)$, and co-rotational (Jaumann) derivative given by $\dot{\boldsymbol{D}}=d \boldsymbol{D} / d t+\boldsymbol{D} \boldsymbol{\Omega}-\boldsymbol{\Omega} \boldsymbol{D}$, with $d / d t=\partial / \partial t+\mathbf{u} \cdot \boldsymbol{\nabla}$. In this form, one recognizes the constitutive form of VCHE or NS- $\alpha$ as a variant of the rate-dependent incompressible homogeneous fluid of second grade [25], [26], whose viscous dissipation, however, is modified by the Helmholtz operator (1 $\alpha^{2} \Delta$ ). There is a tradition at least since Rivlin 27] of modeling turbulence by using continuum mechanics principles such as objectivity and material frame indifference (see also [28]). For example, this sort of approach is taken in deriving Reynolds stress algebraic equation models 29]. Rate-dependent closure models of mean turbulence such as the VCHE or NS- $\alpha$ closure model have also been obtained by the two-scale DIA approach [30] and by the renormalization group methods 31]. We shall see in Section 6 that the covariance $\langle\boldsymbol{\xi} \boldsymbol{\xi}\rangle$ contributes a fluctuation shear stress term in the total stress tensor for the order $O\left(|\boldsymbol{\xi}|^{2}\right)$ compressible Lagrangian mean motion (LMM) model.

\subsection{Comparison of VCHE or NS- $\alpha$ with LES and RANS models.}

Reynolds-averaged Navier-Stokes (RANS) models of turbulence are part of the classic theoretical development of the subject [10], [32], [33]. The related Large Eddy Simulation (LES) turbulence modeling approach [34], [35], [36], provides an operational definition of the intuitive idea of Eulerian resolved scales of motion in 
turbulent flow. In this approach a filtering function $\mathcal{F}(\mathbf{r})$ is introduced and the Eulerian velocity field $\mathbf{U}_{E}$ is filtered in an integral sense, as

$$
\overline{\mathbf{u}}(\mathbf{r}) \equiv \int_{\mathbb{R}^{3}} d^{3} r^{\prime} \mathcal{F}\left(\mathbf{r}-\mathbf{r}^{\prime}\right) \mathbf{U}_{E}\left(\mathbf{r}^{\prime}\right) .
$$

This convolution of $\mathbf{U}_{E}$ with $\mathcal{F}$ defines the large scale, resolved, or filtered velocity, $\overline{\mathbf{u}}$. The corresponding small scale, or subgrid scale velocity, $\mathbf{u}^{\prime}$, is then defined as the difference,

$$
\mathbf{u}^{\prime}(\mathbf{r}) \equiv \mathbf{U}_{E}(\mathbf{r})-\overline{\mathbf{u}}(\mathbf{r}) .
$$

When this filtering operation is applied to the Navier-Stokes system, the following dynamical equation is obtained for the filtered velocity, $\overline{\mathbf{u}}$, cf. equation (5.9),

$$
\frac{\partial}{\partial t} \overline{\mathbf{u}}+\overline{\mathbf{u}} \cdot \boldsymbol{\nabla} \overline{\mathbf{u}}=-\operatorname{div} \overline{\boldsymbol{T}}-\nabla \bar{p}+\nu \Delta \overline{\mathbf{u}}, \quad \boldsymbol{\nabla} \cdot \overline{\mathbf{u}}=0,
$$

in which $\bar{p}$ is the filtered pressure field (required to maintain $\boldsymbol{\nabla} \cdot \overline{\mathbf{u}}=0$ ) and the tensor difference

$$
\overline{\mathbf{T}}=\overline{\left(\mathbf{U}_{E} \mathbf{U}_{E}\right)}-\overline{\mathbf{u}} \overline{\mathbf{u}},
$$

represents the subgrid scale stress due to the turbulent eddies. This subgrid scale stress tensor appears in the same form as the Reynolds stress tensor obtained from Reynolds averaging the Navier-Stokes equation.

The results of Chen et al. [3]- [5], may be given either an LES, or RANS interpretation simply by comparing the constitutive form of the VCHE or NS- $\alpha$ closure model in (5.9) term by term with equation (5.12), provided one may ignore the difference between Eulerian mean, and Lagrangian mean velocities as being of higher order. Additional LES interpretations, discussions and numerical results for forcedturbulence simulations of the VCHE model will be presented elsewhere [37.

\subsection{Comparison of VCHE or NS- $\alpha$ to Leray's equation}

The Leray regularization of the Navier-Stokes equations is given by [38],

$$
\frac{\partial}{\partial t} \mathbf{U}+\langle\mathbf{U}\rangle_{\ell} \cdot \nabla \mathbf{U}=\nu \Delta \mathbf{U}-\nabla p, \quad \boldsymbol{\nabla} \cdot \mathbf{U}=0=\nabla \cdot\langle\mathbf{U}\rangle_{\ell},
$$

in which the velocity field $\mathbf{U}$ is transported by the spatially filtered velocity

$$
\langle\mathbf{U}\rangle_{\ell}(\mathbf{r})=\ell^{-3} \int_{\mathbb{R}^{3}} g\left(\ell^{-1}\left(\mathbf{r}-\mathbf{r}^{\prime}\right)\right) \mathbf{U}\left(\mathbf{r}^{\prime}\right) d^{3} \mathbf{r}^{\prime} .
$$

Here $g \in C^{\infty}\left(\mathbb{R}^{3}\right)$ is a smooth positive function that vanishes outside a finite sphere and is normalized to unity, $\int_{\mathbb{R}^{3}} g=1$. Thus, the spatial scales in $\langle\mathbf{U}\rangle_{\ell}$ smaller than $\ell$ have been smoothly filtered out. The spatially filtered velocity $\langle\mathbf{U}\rangle_{\ell}$ satisfies the important inequality

$$
\left|\langle\mathbf{U}\rangle_{\ell}(\mathbf{r})\right| \leq \max _{\mathbf{r}^{\prime}}\left|\mathbf{U}\left(\mathbf{r}^{\prime}\right)\right|
$$


obtained by taking the sup norm of its definition (5.15) and using the normalization of $g$. Solutions of the Leray equation (5.14) satisfy various regularity properties that are also shared by solutions of the VCHE [39]. The only difference between the forms of these two equations is that the VCHE or NS- $\alpha$ closure model contains the additional term $v_{j} \nabla u^{j}$, stemming from its derivation as an Euler-Poincaré equation. This additional term ensures the Kelvin-Noether circulation theorem for the VCHE. That is, the VCHE or NS- $\alpha$ closure model satisfies

$$
\frac{d}{d t} \oint_{\gamma(\mathbf{u})} \mathbf{v} \cdot d \mathbf{x}=\oint_{\gamma(\mathbf{u})}\left[\frac{\partial \mathbf{v}}{\partial t}+\mathbf{u} \cdot \nabla \mathbf{v}+v_{j} \nabla u^{j}\right] \cdot d \mathbf{x}=\nu \oint_{\gamma(\mathbf{u})} \Delta \mathbf{v} \cdot d \mathbf{x}
$$

for any closed curve $\gamma(\mathbf{u})$ that moves with the Eulerian mean fluid velocity $\mathbf{u}$. In comparison, the Leray equation satisfies

$$
\frac{d}{d t} \oint_{\gamma\left(\langle\mathbf{U}\rangle_{\ell}\right)} \mathbf{U} \cdot d \mathbf{x}=\oint_{\gamma\left(\langle\mathbf{U}\rangle_{\ell}\right)} \mathbf{U} \cdot d\langle\mathbf{U}\rangle_{\ell}+\nu \oint_{\gamma\left(\langle\mathbf{U}\rangle_{\ell}\right)} \Delta \mathbf{U} \cdot d \mathbf{x}
$$

for any closed curve $\gamma\left(\langle\mathbf{U}\rangle_{\ell}\right)$ that moves with the filtered fluid velocity $\langle\mathbf{U}\rangle_{\ell}$. Thus, the Leray equation has an additional source of circulation arising from the difference between its filtered and unfiltered velocities, while the VCHE or NS- $\alpha$ closure model does not have such a term.

This difference reappears in the vorticity dynamics for the two theories. Namely, upon using incompressibility $\boldsymbol{\nabla} \cdot \mathbf{u}=0$ we have

$$
\frac{\partial \mathbf{q}}{\partial t}+\mathbf{u} \cdot \nabla \mathbf{q}=\mathbf{q} \cdot \nabla \mathbf{u}+\nu \Delta \mathbf{q}, \quad \text { where } \quad \mathbf{q} \equiv \operatorname{curl} \mathbf{v}, \text { for VCHE },
$$

and

$$
\begin{aligned}
\frac{\partial \boldsymbol{\omega}}{\partial t}+\langle\mathbf{U}\rangle_{\ell} \cdot \boldsymbol{\nabla} \boldsymbol{\omega}= & \boldsymbol{\omega} \cdot \boldsymbol{\nabla}\langle\mathbf{U}\rangle_{\ell}+\nu \Delta \boldsymbol{\omega} \text { for Leray's equation } \\
& +\left[\boldsymbol{\nabla} U_{j} \times \nabla\left\langle U^{j}\right\rangle_{\ell}\right], \text { where } \boldsymbol{\omega} \equiv \operatorname{curl} \mathbf{U},
\end{aligned}
$$

and we have used incompressibility of the filtered velocity, $\langle\mathbf{U}\rangle_{\ell}$. Thus, the right hand side of the vorticity dynamics for Leray's equation contains an additional source term, compared to the curl of the VCHE or NS- $\alpha$ equation.

Outlook for the remainder of the paper. We shall apply the results of Holm, Marsden and Ratiu [1], [2], to recast the Lagrangian mean motion (LMM) model and the order $O\left(|\boldsymbol{\xi}|^{2}\right)$ compressible model into the Euler-Poincaré framework. EulerPoincaré systems are the Lagrangian version of Lie-Poisson Hamiltonian systems. Reformulating the LMM equations this way facilitates their Eulerian analysis, e.g., by providing their Kelvin-Noether circulation theorem, as well as energy and momentum conservation as part of a general framework. We shall then use the equations of the LMM model in making a natural adaptation and development of the turbulence modeling results of Chen et al. [3]- [5]. Namely, we shall formulate a second moment closure model for turbulence based on adding a certain viscosity 
term to the ideal LMM equations. This Lagrangian mean turbulence closure model will then be adapted to include rotation and stratification for potential applications in geophysical problems. Lower-dimensional examples of Lagrangian mean theories will also be considered and then we shall turn our attention to developing Eulerian mean theories using the Euler-Poincaré framework, as well.

\section{Second moment closure equations}

\subsection{Euler-Poincaré formulation}

We recall that the variables in the Lagrangian mean theory with averaged approximate Lagrangian $\langle L\rangle$ in equation (3.34) are the Lagrangian mean velocity $\mathbf{u}$ and the advected, or "frozen-in" quantities $D$ and $\left\langle\xi^{k} \xi^{l}\right\rangle$ : the volume element and the Lagrangian mean covariance of the fluctuating displacement. Such quantities satisfy a certain Lie-derivative relation [1], such as the continuity equation (2.7) for the volume element $D$,

$$
0=\left.\frac{\partial}{\partial t}\right|_{\mathbf{a}}\left(d^{3} a\right)=\left(\frac{\partial}{\partial t}+£_{\mathbf{u}}\right)\left(D d^{3} x\right)=\left(\frac{\partial D}{\partial t}+\nabla \cdot(D \mathbf{u})\right)\left(d^{3} x\right),
$$

where $£_{\mathbf{u}}$ denotes Lie derivative with respect to the Lagrangian mean fluid velocity, $\mathbf{u}(\mathbf{x}, t)$. There is also the geometrical relation (3.17), or its equivalent commutator form (3.25), for the slow time evolution of the fluctuation components $\xi^{k}$, for $k=$ $1,2,3$. Namely,

$$
0=\left.\frac{\partial}{\partial t}\right|_{\mathbf{a}}\left(\tilde{\xi}^{A} \frac{\partial}{\partial a^{A}}\right)=\left(\frac{\partial}{\partial t}+£_{\mathbf{u}}\right)\left(\xi^{k} \frac{\partial}{\partial x^{k}}\right)=\left(\frac{\partial \xi^{k}}{\partial t}+u^{j} \xi_{, j}^{k}-\xi^{j} u_{, j}^{k}\right) \frac{\partial}{\partial x^{k}} .
$$

This equation for $\xi^{k}$ implies a geometrical relation of the same type for all the statistical moments $\left\langle\xi^{k} \xi^{l} \ldots \xi^{m}\right\rangle$. In particular, the second moment $\left\langle\xi^{k} \xi^{l}\right\rangle$ (the Lagrangian mean covariance of the fluctuations) satisfies, cf. equation (3.41),

$$
\begin{aligned}
0 & =\left.\frac{\partial}{\partial t}\right|_{\mathbf{a}}\left(\left\langle\tilde{\xi}^{A} \tilde{\xi}^{B}\right\rangle \frac{\partial}{\partial a^{A}} \otimes \frac{\partial}{\partial a^{B}}\right)=\left(\frac{\partial}{\partial t}+£_{\mathbf{u}}\right)\left(\left\langle\xi^{k} \xi^{l}\right\rangle \frac{\partial}{\partial x^{k}} \otimes \frac{\partial}{\partial x^{l}}\right) \\
& =\left(\frac{\partial}{\partial t}\left\langle\xi^{k} \xi^{l}\right\rangle+u^{j}\left\langle\xi^{k} \xi^{l}\right\rangle_{, j}-\left\langle\xi^{j} \xi^{l}\right\rangle u_{, j}^{k}-\left\langle\xi^{k} \xi^{j}\right\rangle u_{, j}^{l}\right) \frac{\partial}{\partial x^{k}} \otimes \frac{\partial}{\partial x^{l}} .
\end{aligned}
$$

In vector notation this equation for the Lagrangian mean covariance dynamics is, cf. equation (3.42),

$$
\frac{d}{d t}\langle\xi \xi\rangle=\langle\xi \xi\rangle \cdot \nabla \mathbf{u}+\nabla \mathbf{u}^{\mathrm{T}} \cdot\langle\boldsymbol{\xi} \boldsymbol{\xi}\rangle
$$

Thus, the Taylor hypothesis (3.17) provides an approximate equation for the evolution of the fluctuation statistical moments; in particular, for their Lagrangian mean covariance. The Eulerian components of this equation can be rewritten as

$$
\frac{\partial}{\partial t}\left\langle\xi^{k} \xi^{l}\right\rangle=\left(-\left\langle\xi^{k} \xi^{l}\right\rangle_{, j}+\left\langle\xi^{a} \xi^{l}\right\rangle \partial_{a} \delta_{j}^{k}+\left\langle\xi^{k} \xi^{a}\right\rangle \partial_{a} \delta_{j}^{l}\right) u^{j},
$$


in which the right hand side is expressed as a differential operator acting on $u^{j}(\mathbf{x}, t)$. This operator will reappear in the Lie-Poisson Hamiltonian formulation of the ideal second moment equations in Section 7 . Note that the isotropic, homogeneous initial condition $\left\langle\xi^{k} \xi^{l}\right\rangle=\delta^{k l}$ is not invariant under the dynamics of equation (6.6) for nontrivial velocity shear. Thus, when shear is present, the Lagrangian mean covariance of the fluctuations will not remain isotropic and homogeneous under the LMM dynamics, even if it were initially so.

Using results of Holm, Marsden and Ratiu [1], we compute the Euler-Poincaré equation for the Lagrangian $\langle L\rangle\left(\mathbf{u}, D,\left\langle\xi^{k} \xi^{l}\right\rangle\right)$ depending on the Lagrangian mean velocity $\mathbf{u}$, and advected quantities $D$ and $\left\langle\xi^{k} \xi^{l}\right\rangle$. This Euler-Poincaré equation is given by the following extension of equation (2.11),

$$
\begin{aligned}
0= & \left(\frac{\partial}{\partial t}+u^{j} \frac{\partial}{\partial x^{j}}\right) \frac{1}{D} \frac{\delta\langle L\rangle}{\delta u^{i}}+\frac{1}{D} \frac{\delta\langle L\rangle}{\delta u^{j}} u_{, i}^{j}-\left(\frac{\delta\langle L\rangle}{\delta D}\right)_{, i} \\
& +\frac{1}{D}\left[\frac{\delta\langle L\rangle}{\delta\left\langle\xi^{k} \xi^{l}\right\rangle}\left\langle\xi^{k} \xi^{l}\right\rangle_{, i}+\left(\frac{\delta\langle L\rangle}{\delta\left\langle\xi^{i} \xi^{l}\right\rangle}\left\langle\xi^{k} \xi^{l}\right\rangle\right)_{, k}+\left(\frac{\delta\langle L\rangle}{\delta\left\langle\xi^{k} \xi^{i}\right\rangle}\left\langle\xi^{k} \xi^{l}\right\rangle\right)_{, l}\right] .
\end{aligned}
$$

Thus, the additional advected quantities $\left\langle\xi^{k} \xi^{l}\right\rangle$ in general contribute their own reactive forces, appearing in the second line of the equation of motion (6.7). We compute the following variational derivatives of the averaged approximate Lagrangian $\langle L\rangle$ in equation (3.34)

$$
\begin{aligned}
\frac{1}{D} \frac{\delta\langle L\rangle}{\delta \mathbf{u}} & =\mathbf{u}-\frac{1}{D}\left(\partial_{k} D\left\langle\xi^{k} \xi^{l}\right\rangle \partial_{l}\right) \mathbf{u} \equiv \mathbf{v}, \\
\frac{\delta\langle L\rangle}{\delta D} & =-p+\frac{1}{2}|\mathbf{u}|^{2}+\frac{1}{2}\left\langle\xi^{k} \xi^{l}\right\rangle\left(\mathbf{u}_{, k} \cdot \mathbf{u}_{, l}\right) \equiv-P_{t o t}, \\
\frac{\delta\langle L\rangle}{\delta p} & =1-D, \\
\frac{\delta\langle L\rangle}{\delta\left\langle\xi^{k} \xi^{l}\right\rangle} & =\frac{D}{2}\left(\mathbf{u}_{, k} \cdot \mathbf{u}_{, l}\right) .
\end{aligned}
$$

Consequently, the Lagrangian mean Euler-Poincaré equation (5.7) for this averaged Lagrangian (after setting $D=1$ ) takes the form,

$$
\begin{gathered}
\frac{\partial v_{i}}{\partial t}+u^{j} v_{i, j}+v_{j} u_{, i}^{j}=\left(-p+\frac{1}{2}|\mathbf{u}|^{2}+\frac{1}{2}\left\langle\xi^{k} \xi^{l}\right\rangle\left(\mathbf{u}_{, k} \cdot \mathbf{u}_{, l}\right)\right)_{, i} \\
-\frac{1}{2}\left[\left(\mathbf{u}_{, k} \cdot \mathbf{u}_{, l}\right)\left\langle\xi^{k} \xi^{l}\right\rangle_{, i}+\left(\left(\mathbf{u}_{, i} \cdot \mathbf{u}_{, l}\right)\left\langle\xi^{k} \xi^{l}\right\rangle\right)_{, k}+\left(\left(\mathbf{u}_{, k} \cdot \mathbf{u}_{, i}\right)\left\langle\xi^{k} \xi^{l}\right\rangle\right)_{, l}\right] \\
\text { where } \quad v_{i}=u_{i}-\left(\partial_{k}\left\langle\xi^{k} \xi^{l}\right\rangle \partial_{l}\right) u_{i} \quad \text { and } \quad u_{, i}^{i}=0
\end{gathered}
$$

Again one sees in the entire second line of equation (6.9) the reactive forces arising from the variations of the Lagrangian with respect to the covariance $\left\langle\xi^{k} \xi^{l}\right\rangle$.

Contrasting the Euler-Poincaré equation (6.9) with the CH equation. In the present case, the advected second moments $\left\langle\xi^{k} \xi^{l}\right\rangle$ for the full system satisfy 
their dynamical equation (6.5). If isotropic, homogeneous statistics were prescribed instead, so that $\left\langle\xi^{k} \xi^{l}\right\rangle=\alpha^{2} \delta^{k l}$ with constant $\alpha^{2}$, then both equation (6.5) and the entire second line in the motion equation $(6.9)$ would be absent, (since the corresponding variations in $\left\langle\xi^{k} \xi^{l}\right\rangle$ would not be taken in this case) and the motion equation $(6.9)$ of the Lagrangian mean model then would return to the $n$-dimensional generalization of the $\mathrm{CH}$ equation, i.e., the set (5.1) - (5.4) introduced in [1], [2]. Note, however, that the $n$-dimensional $\mathrm{CH}$ equation set is not an invariant subsystem of the Euler-Poincaré system (6.9), with definition (6.10) and advection law (6.5), because (as mentioned earlier) the initial condition $\left\langle\xi^{k} \xi^{l}\right\rangle=\delta^{k l}$ is not invariant under the dynamics of equation (6.5) for nontrivial velocity shear. This means that the Lagrangian mean model is a departure from the VCHE model, rather than being an extension of it. We shall return to this matter in Section 12, when we discuss Eulerian mean fluid models.

\subsection{Momentum conservation - stress tensor formulation}

Noether's theorem guarantees there is a conserved momentum for the Euler-Poincaré equations (6.9), since the averaged approximate Lagrangian $\langle L\rangle$ in equation (3.34) has no explicit spatial dependence. Moreover, the integrand $\mathcal{L}$ in this Lagrangian is a polynomial in the Lagrangian mean velocity $\mathbf{u}$, its gradient $\mathbf{u}_{, k}$, and the advected quantities $D$ and $\left\langle\xi^{k} \xi^{l}\right\rangle$. That is,

$$
\langle L\rangle=\int d^{3} x \mathcal{L}\left(\mathbf{u}, \mathbf{u}_{, k}, D,\left\langle\xi^{k} \xi^{l}\right\rangle\right),
$$

with $\mathcal{L}$ a polynomial function of its arguments. In this case, we may express the Lagrangian mean Euler-Poincaré equations (6.7) in the momentum conservation form,

$$
\frac{\partial m_{i}}{\partial t}=-\frac{\partial}{\partial x^{j}} T_{i}^{j}
$$

with momentum density components $m_{i}, i=1,2,3$ defined by

$$
m_{i} \equiv \frac{\delta\langle L\rangle}{\delta u^{i}}=\frac{\partial \mathcal{L}}{\partial u^{i}}-\frac{\partial}{\partial x^{k}}\left(\frac{\partial \mathcal{L}}{\partial u_{, k}^{i}}\right)
$$

and stress tensor $T_{i}^{j}$ given by

$$
\begin{aligned}
T_{i}^{j}= & m_{i} u^{j}-\frac{\partial \mathcal{L}}{\partial u_{, j}^{k}} u_{, i}^{k}+\frac{\partial \mathcal{L}}{\partial\left\langle\xi^{i} \xi^{l}\right\rangle}\left\langle\xi^{j} \xi^{l}\right\rangle+\frac{\partial \mathcal{L}}{\partial\left\langle\xi^{k} \xi^{i}\right\rangle}\left\langle\xi^{k} \xi^{j}\right\rangle \\
& +\delta_{i}^{j}\left(\mathcal{L}-D \frac{\partial \mathcal{L}}{\partial D}\right) .
\end{aligned}
$$

Equation (6.12) then implies conservation of the domain-integrated momentum, $\int \mathbf{m} d^{3} x$, provided the normal component of the stress tensor $T_{i}^{j}$ vanishes on the boundary. 
In our particular case, expression (6.14) for the stress tensor $T_{i}^{j}$ simplifies remarkably, to become

$$
T_{i}^{j}=m_{i} u^{j}+p \delta_{i}^{j}, \quad \text { where }\left.\quad m_{i}\right|_{D=1}=v_{i} \equiv u_{i}-\left(\partial_{k}\left\langle\xi^{k} \xi^{l}\right\rangle \partial_{l}\right) u_{i} .
$$

Consequently, the equivalent Euler-Poincaré motion equation (6.9) simplifies to

$$
\frac{\partial v_{i}}{\partial t}=-\frac{\partial}{\partial x^{j}}\left(v_{i} u^{j}+p \delta_{i}^{j}\right) .
$$

The ideal Lagrangian mean motion (LMM) model. Using incompressibility $\left(u_{, j}^{j}=0\right)$ reduces the expression (6.16) for momentum conservation to the $\boldsymbol{L a}$ grangian mean motion (LMM) equation for an ideal fluid, cf. equation (3.58),

$$
\begin{aligned}
& \frac{\partial \mathbf{v}}{\partial t}+\mathbf{u} \cdot \boldsymbol{\nabla} \mathbf{v}=-\nabla p, \quad \boldsymbol{\nabla} \cdot \mathbf{u}=0, \\
& \text { where }\left.\quad \mathbf{v} \equiv \frac{1}{D} \frac{\delta\langle L\rangle}{\delta \mathbf{u}}\right|_{D=1}=\left(1-\partial_{k}\left\langle\xi^{k} \xi^{l}\right\rangle \partial_{l}\right) \mathbf{u} .
\end{aligned}
$$

The boundary conditions are recalled from equations (4.14), 4.15) and (5.5) as

$$
\mathbf{v} \cdot \hat{\boldsymbol{n}}=0, \quad \mathbf{u}=0, \quad \text { and } \quad\langle\boldsymbol{\xi} \boldsymbol{\xi}\rangle \cdot \hat{\boldsymbol{n}}=0, \quad \text { on a fixed boundary } .
$$

In $(6.17)$ the continuity equation, $\boldsymbol{\nabla} \cdot \mathbf{u}=0$, allows the Lagrangian mean pressure $p$ to be determined from an elliptic equation, by virtue of the commutation relation (3.49). The auxiliary equation (6.5) for the slow time dynamics of the advected Lagrangian mean covariance may be rewritten as,

$$
\left(\frac{\partial}{\partial t}+\mathbf{u} \cdot \boldsymbol{\nabla}\right)\left\langle\xi^{k} \xi^{l}\right\rangle=\left\langle\xi^{j} \xi^{l}\right\rangle u_{, j}^{k}+\left\langle\xi^{k} \xi^{j}\right\rangle u_{, j}^{l}
$$

This equation updates the Lagrangian mean covariance $\left\langle\xi^{k} \xi^{l}\right\rangle$ and, thus, closes the LMM system.

Contrasting the LMM model with the Euler equations. Remarkably, the reaction forces due to the fluctuations in the Euler-Poincare motion equation (6.9) exactly cancel the contributions of the fluctuations in the pressure and line-element stretching term $\left(v_{j} u_{, i}^{j}\right)$. This cancellation leaves only one main effect: the fluid parcels in the LMM model are transported by velocity $\mathbf{u}$ instead of $\mathbf{v}$. Had we made no approximation at all in our Lagrangian $L(\omega)$ before averaging, we would have gotten exact cancellation of all of the fluctuational effects and returned entirely to Euler's equations.

So, instead of returning us entirely to the Euler equations, the Taylor series approximation we made in Section 3.3 before averaging the Lagrangian $L(\omega)$ produces one essential difference between the LMM model in equations (6.17) - 6.20) and 
the original Euler equations (2.14). Namely, the transport velocity $\mathbf{u}$ is smoothed relative to $\mathbf{v}$, by inversion of the dynamical Helmholtz operator,

$$
\mathbf{u}=(1-\tilde{\Delta})^{-1} \mathbf{v} \quad \text { where } \quad 1-\tilde{\Delta}=1-\partial_{k}\left\langle\xi^{k} \xi^{l}\right\rangle \partial_{l},
$$

whose statistical 'metric' $\left\langle\xi^{k} \xi^{l}\right\rangle$ changes and adapts dynamically according to equation (6.20) as the fluid moves. Smoothing the transport velocity to improve the mathematical properties of the incompressible fluid equations has been studied previously, going back at least to the work of Leray [38] in the 1930s, as we discussed earlier. However, the adaptive smoothing introduced by inverting the dynamical Helmholtz operator (6.21) is new here, as far as we know. Euler's equations are recovered as an invariant subsystem of the LMM equation set for $\langle\boldsymbol{\xi} \xi\rangle$ identically zero.

We recall the remarkable commutation relation (3.49)

$$
\left[\frac{d}{d t}, \tilde{\Delta}_{D}\right]=0, \quad \text { where } \quad \tilde{\Delta}_{D} \equiv D^{-1} \partial_{k} D\left\langle\xi^{k} \xi^{l}\right\rangle \partial_{l},
$$

which may also be verified directly from the continuity equation (2.7) and the Lagrangian mean covariance equation $(\underline{6.6})$. Consequently, since $\left.\tilde{\Delta}_{D}\right|_{D=1} \equiv \tilde{\Delta}$, the ideal LMM motion equation (6.17) may be rewritten alternatively as

$$
\frac{\partial \mathbf{u}}{\partial t}+\mathbf{u} \cdot \nabla \mathbf{u}=-(1-\tilde{\Delta})^{-1} \nabla p, \quad \nabla \cdot \mathbf{u}=0 .
$$

Therefore, the effect of the advected fluctuations in this alternative representation of the LMM equation is simply to smooth the pressure gradient in an adaptive fashion depending on the velocity shear, through the dynamics of the covariance $\langle\boldsymbol{\xi} \xi\rangle$.

As guaranteed in advance by the mathematical theory developed in in [1], the Euler-Poincaré equation (6.17) with definition (6.18) for the LMM model is equivalent to the Euler-Lagrange equation (3.44). Because of the commutation property (6.22), there is also an equivalent alternative form of the LMM motion equation, given in (6.23). This alternative, but equivalent, form of the motion equation for LMM provides alternative interpretations of the effects of the fluctuation covariance on the Lagrangian mean motion.

\subsection{Momentum conservation for the order $O\left(|\xi|^{2}\right)$ model}

We recall the averaged approximate Lagrangian (3.34) in Eulerian form,

$\langle L\rangle=\int d^{3} x\left\{\frac{D}{2}\left[|\mathbf{u}|^{2}+\left\langle\xi^{k} \xi^{l}\right\rangle\left(\mathbf{u}_{, k} \cdot \mathbf{u}_{, l}\right)\right]+\left(p-\frac{1}{2} \frac{\partial^{2} p}{\partial x^{k} \partial x^{l}}\left\langle\xi^{k} \xi^{l}\right\rangle\right)-p D\right\}$.

Note that $p$ is the Lagrangian mean pressure and according to equation (4.13) the quantity in parentheses,

$$
P_{E} \equiv p-\frac{1}{2} \frac{\partial^{2} p}{\partial x^{k} \partial x^{l}}\left\langle\xi^{k} \xi^{l}\right\rangle
$$


is the Eulerian mean pressure. The momentum density components $m_{i}, i=$ 1,2,3 for this Lagrangian are

$$
m_{i} \equiv \frac{\delta\langle L\rangle}{\delta u^{i}}=D\left(1-\tilde{\Delta}_{D}\right) u_{i}
$$

where $\tilde{\Delta}_{D}$ satisfies 3.49 and

$$
D=1-\frac{1}{2}\left\langle\xi^{i} \xi^{l}\right\rangle_{, k l}
$$

The stress tensor $T_{i}^{j}$ is obtained by using equation (6.14) as

$$
T_{i}^{j}=m_{i} u^{j}-\frac{1}{2} \frac{\partial^{2} p}{\partial x^{i} \partial x^{l}}\left\langle\xi^{j} \xi^{l}\right\rangle+\delta_{i}^{j}\left(p-\frac{1}{2} \frac{\partial^{2} p}{\partial x^{k} \partial x^{l}}\left\langle\xi^{k} \xi^{l}\right\rangle\right) .
$$

The off-diagonal components $-\frac{1}{2} p_{, i l}\left\langle\xi^{j} \xi^{l}\right\rangle$ may be regarded as a fluctuation stress tensor. Using the continuity equation (2.7) for $D$ transforms the momentum conservation law (6.12) into the equivalent motion equation for the LMM model with second order compressibility, cf. equation (3.50),

$$
\begin{aligned}
\left(\frac{\partial}{\partial t}+\mathbf{u} \cdot \boldsymbol{\nabla}\right)\left(1-\tilde{\Delta}_{D}\right) u_{i} & =-\frac{1}{D} \frac{\partial P_{E}}{\partial x^{i}}+\frac{1}{D} \frac{\partial}{\partial x^{j}}\left(\frac{\partial^{2} p}{\partial x^{i} \partial x^{l}}\left\langle\xi^{j} \xi^{l}\right\rangle\right) \\
\text { with } \boldsymbol{\nabla} \cdot \mathbf{u} & =-\frac{1}{D} \frac{d D}{d t}=\frac{1}{2} \frac{d}{d t}\left\langle\xi^{i} \xi^{l}\right\rangle_{, k l}+O\left(|\boldsymbol{\xi}|^{4}\right)
\end{aligned}
$$

The boundary conditions for this model are the same as in (6.19), namely,

$$
\mathbf{v} \cdot \hat{\boldsymbol{n}}=0, \quad \mathbf{u}=0, \quad \text { and } \quad\langle\boldsymbol{\xi} \boldsymbol{\xi}\rangle \cdot \hat{\boldsymbol{n}}=0, \quad \text { on a fixed boundary }
$$

This completes the transformation of the Lagrangian form (3.44) of this motion equation to its equivalent Eulerian form. As guaranteed by the Euler-Poincaré approach, the equivalence of equations (3.44) and (6.29) may also be verified by a direct calculation.

\subsection{Kelvin circulation theorem for the Lagrangian mean model}

Being Euler-Poincaré, the incompressible LMM equation (6.17) has a corresponding Kelvin-Noether circulation theorem. Namely, this equation implies

$$
\frac{d}{d t} \oint_{\gamma(\mathbf{u})} \mathbf{v} \cdot d \mathbf{x}=\oint_{\gamma(\mathbf{u})}\left[\frac{\partial \mathbf{v}}{\partial t}+\mathbf{u} \cdot \nabla \mathbf{v}+v_{j} \boldsymbol{\nabla} u^{j}\right] \cdot d \mathbf{x}=\oint_{\gamma(\mathbf{u})} \mathbf{v} \cdot d \mathbf{u}
$$

for any closed curve $\gamma(\mathbf{u})$ that moves with the Lagrangian mean fluid velocity $\mathbf{u}$. This expression for the Kelvin-Noether property of the Lagrangian mean motion equation in $3 \mathrm{D}$ is reminiscent of corresponding expressions in wave, mean-flow interaction theory [14]. See also the Leray equation result (5.18). The main point is that the presence of the fluctuation covariance $\left\langle\xi^{k} \xi^{l}\right\rangle$ creates circulation of the total specific momentum $\mathbf{v}=\mathbf{u}-\left(\partial_{k}\left\langle\xi^{k} \xi^{l}\right\rangle \partial_{l}\right) \mathbf{u}$. 
Alternatively, from equation (6.23) we may write another Kelvin-Noether circulation theorem, namely

$$
\frac{d}{d t} \oint_{\gamma(\mathbf{u})} \mathbf{u} \cdot d \mathbf{x}=-\oint_{\gamma(\mathbf{u})}\left((1-\tilde{\Delta})^{-1} \nabla p\right) \cdot d \mathbf{x}
$$

which represents the circulation dynamics of $\mathbf{u}$, rather than $\mathbf{v}$. Thus, the fluctuation covariance creates circulation of both $\mathbf{u}$ and $\mathbf{v}$.

\subsection{Vortex stretching equation for the Lagrangian mean model}

In three dimensions, we may use a vector identity to re-express the LMM equation 6.17) in its equivalent "curl" form, as

$$
\frac{\partial}{\partial t} \mathbf{v}-\mathbf{u} \times(\boldsymbol{\nabla} \times \mathbf{v})+\nabla p+u^{j} \nabla v_{j}=0, \quad \nabla \cdot \mathbf{u}=0 .
$$

The curl of this equation in turn yields a transport and creation equation for the

Lagrangian mean vorticity, $\mathrm{q} \equiv \operatorname{curl} \mathrm{v}$,

$$
\frac{\partial \mathbf{q}}{\partial t}+\mathbf{u} \cdot \nabla \mathbf{q}=\mathbf{q} \cdot \nabla \mathbf{u}+\left[\boldsymbol{\nabla} v_{j} \times \nabla u^{j}\right], \quad \text { where } \quad \mathbf{q} \equiv \operatorname{curl} \mathbf{v},
$$

and we have used incompressibility of $\mathbf{u}$. Thus, $\mathbf{u}$ is the transport velocity for the generalized vorticity $\mathbf{q}$ and the expected vortex stretching term $\mathbf{q} \cdot \nabla \mathbf{u}$ is accompanied by an additional vortex creation term, $\boldsymbol{\nabla} v_{j} \times \nabla u^{j}$. Of course, this additional term is also responsible for the creation of circulation of $\mathbf{v}$ in the KelvinNoether circulation theorem (6.32). In particular, Stokes' theorem and equation 6.32) imply

$$
\frac{d}{d t} \iint_{S(\mathbf{u})} \operatorname{curl} \mathbf{v} \cdot d \mathbf{S}=\iint_{S(\mathbf{u})} d v_{j} \wedge d u^{j}=\iint_{S(\mathbf{u})}\left[\boldsymbol{\nabla} v_{j} \times \nabla u^{j}\right] \cdot d \mathbf{S},
$$

where the curve $\gamma(\mathbf{u})$ is the boundary of the surface $S(\mathbf{u})$. One may compare this result with the Leray case, in equation (5.20).

Alternatively, from equation (6.23) we may write another form of the vorticity dynamics, for curl $\mathbf{u}$ rather than curl $\mathbf{v}$, namely

$$
\frac{\partial \boldsymbol{\omega}}{\partial t}+\mathbf{u} \cdot \boldsymbol{\nabla} \boldsymbol{\omega}=\boldsymbol{\omega} \cdot \boldsymbol{\nabla} \mathbf{u}-\boldsymbol{\nabla} \times\left((1-\tilde{\Delta})^{-1} \boldsymbol{\nabla} p\right) \text { where } \boldsymbol{\omega} \equiv \operatorname{curl} \mathbf{u},
$$

and we have used incompressibility of the Lagrangian mean velocity $\mathbf{u}$.

\subsection{Energetics of the Lagrangian mean model}

The sum of the inner products of $\mathbf{v}$ with equation (6.23) and $\mathbf{u}$ with equation (6.34) yields conservation of energy,

$$
E=\frac{1}{2} \int d^{3} x\left(|\mathbf{u}|^{2}+\left\langle\xi^{k} \xi^{l}\right\rangle \mathbf{u}_{, k} \cdot \mathbf{u}_{, l}\right)=\frac{1}{2} \int d^{3} x \mathbf{u} \cdot \mathbf{v}
$$


after integrating by parts, using incompressibility and applying the boundary conditions (6.19). Naturally, this energy is also conserved in $n$ dimensions. Thus, the covariance of the fluctuations couples to the gradients of the Lagrangian mean velocity. So it costs the system energy either to increase these gradients, or to increase the covariance. Moreover, there is a direct feedback between $\boldsymbol{\nabla u}$ and the dynamics of $\langle\boldsymbol{\xi} \boldsymbol{\xi}\rangle$ given in equation (6.20). We shall see in Section 7 that Legendre transforming the Lagrangian $\langle L\rangle$ in (3.34) gives the following Hamiltonian (still expressed in terms of the velocity $\mathbf{u}$, instead of the momentum density $\mathbf{m}=\delta\langle L\rangle / \delta \mathbf{u}=D \mathbf{v}$ ),

$$
H=\int_{\mathcal{M}} d^{n} x\left[\frac{D}{2}\left(|\mathbf{u}|^{2}+\left\langle\xi^{k} \xi^{l}\right\rangle \mathbf{u}_{, k} \cdot \mathbf{u}_{, l}\right)+p(D-1)\right]
$$

Remark on the geodesic property of the LMM model. When evaluated on the constraint manifold $D=1$, the Lagrangian in (3.34) and the Hamiltonian in (6.39) for the Lagrangian mean motion equation (6.17) coincide in $n$ dimensions. This is expected for a stationary principle giving rise to geodesic motion. The interpretation of the LMM model as describing geodesic motion on the volume preserving diffeomorphism group with respect to the $H_{1}$ metric given by (6.38) will be discussed elsewhere [6].

Conservation properties of the Lagrangian mean covariance. We recall equation (6.5) for $\langle\xi \xi\rangle$,

$$
\frac{d}{d t}\langle\boldsymbol{\xi} \boldsymbol{\xi}\rangle=\left\langle\boldsymbol{\xi} \boldsymbol{\xi} \cdot \nabla \mathbf{u}+\nabla \mathbf{u}^{\mathrm{T}} \cdot\langle\boldsymbol{\xi} \boldsymbol{\xi}\rangle\right.
$$

Since $\operatorname{tr}(\boldsymbol{\nabla u})=0$, the Lagrangian mean covariance $\langle\boldsymbol{\xi} \boldsymbol{\xi}\rangle$ must always have an instantaneously growing direction, along at least one principal axis of the velocity shear tensor, $\boldsymbol{\nabla} \mathbf{u}$. So, $\langle\boldsymbol{\xi} \boldsymbol{\xi}\rangle$ might be systematically growing with time. However, there are limits to this growth. In particular, equation (3.38) shows that this growth in the incompressible case must preserve the value of the determinant det $\langle\boldsymbol{\xi} \boldsymbol{\xi}\rangle$ along flow lines. Thus, stretching and rotation may occur, but for an incompressible flow the volume of the ellipsoid composed of the principle axes of the symmetric covariance tensor $\langle\boldsymbol{\xi} \xi\rangle$ must be preserved on fluid parcels. In the Eulerian representation, equation (3.38) implies

$$
\frac{d}{d t}\left(D^{2} \operatorname{det}\langle\xi \xi\rangle\right)=0, \quad \text { and } \quad \frac{d D}{d t}=0 \quad \text { for } \quad \boldsymbol{\nabla} \cdot \mathbf{u}=0
$$

This, in turn, implies conservation of the following quantity, for any function $\Phi$,

$$
C_{\Phi}=\int_{\mathcal{M}} d^{n} x D \Phi\left(D^{2} \operatorname{det}\langle\boldsymbol{\xi} \boldsymbol{\xi}\rangle\right), \quad \forall \Phi
$$

in which we may set $D=1$ for incompressible flow.

Conservation of the energy in equation (6.38) also has some indications for controlling the Lagrangian mean covariance. Of course, this growth cannot continue indefinitely in the same direction and still satisfy conservation of energy $E$ in (6.38) 
in regions of nontrivial shear. So the conservation of energy must also eventually saturate this potential growth in Lagrangian mean covariance. Thus, from the viewpoint of energetics, while $\langle\boldsymbol{\xi} \boldsymbol{\xi}\rangle$ must always have a direction in which it is growing, this growth must also occur to preserve the determinant $\operatorname{det}\langle\boldsymbol{\xi} \xi\rangle$ and to limit the velocity shear $\boldsymbol{\nabla u}$ in accordance with the available energy in equation (6.38). Therefore, the direction of growth (along the instantaneous principle axes of $\boldsymbol{\nabla} \mathbf{u}$ corresponding to its positive eigenvalues) will keep changing, because of the energetic coupling and direct feedback between $\boldsymbol{\nabla} \mathbf{u}$ and $\langle\boldsymbol{\xi} \boldsymbol{\xi}\rangle$. A dissipative modification of the $\langle\boldsymbol{\xi} \boldsymbol{\xi}\rangle$ dynamics that allows relaxation to the homogeneous isotropic conditions of the VCHE model was introduced in equation (4.36). This modification may be used, in the event that the growth of $\langle\xi \xi\rangle$ should require additional control, e.g., in numerical simulations.

\section{$7 \quad$ Hamiltonian structure of the Lagrangian mean model}

Being Euler-Poincaré, the LMM system consisting of the motion equation (6.9), the continuity equation (2.7) and the Lagrangian mean covariance equation (6.6) must also be a Lie-Poisson Hamiltonian system. This may be verified using standard methods, see, e.g., [1]. The corresponding Lie-Poisson bracket is dual to the semidirect product Lie algebra

$$
\mathfrak{g}=\mathfrak{u} S\left(\Lambda^{0} \oplus S\right),
$$

consisting of vector fields $\eta \in \mathfrak{u}$ acting from the right on the direct sum of functions $f \in \Lambda^{0}$ and metrics $g \in S$. The semidirect product Lie algebra bracket has the expression

$$
\left[\left(\eta_{1}, f_{1}, g_{1}\right),\left(\eta_{2}, f_{2}, g_{2}\right)\right]=\left(\left[\eta_{1}, \eta_{2}\right], f_{1} \eta_{2}-f_{2} \eta_{1}, g_{1} \eta_{2}-g_{2} \eta_{1}\right)
$$

where we denote the induced action of $\mathfrak{u}$ on $\left(\Lambda^{0} \oplus S\right)$ from the right by concatenation, as in $f_{1} \eta_{2}$. Dual coordinates are: $\mathbf{m}=\delta\langle L\rangle / \delta \mathbf{u}=D \mathbf{v}$ dual to $\mathfrak{u} ; D$ dual to $\Lambda^{0}$; and $\left\langle\xi^{k} \xi^{l}\right\rangle$ dual to $S$. The Legendre transformation of the averaged approximate Lagrangian $\langle L\rangle$ in (3.34) produces the Hamiltonian,

$$
H=\int d^{3} x\left[\frac{1}{2} \mathbf{m} \cdot\left(D-\partial_{k} D\left\langle\xi^{k} \xi^{l}\right\rangle \partial_{l}\right)^{-1} \mathbf{m}+p(D-1)\right],
$$

whose proper definition requires defining the inverse of the generalized Helmholtz operator, $\left(D-\partial_{k} D\left\langle\xi^{k} \xi^{l}\right\rangle \partial_{l}\right)$ and using the boundary conditions (6.19). The variational derivatives of this Hamiltonian are given by

$$
\begin{gathered}
\delta H=\int d^{3} x\left[\mathbf{u} \cdot \delta \mathbf{m}-\left(p-\frac{1}{2}|\mathbf{u}|^{2}-\frac{1}{2}\left\langle\xi^{k} \xi^{l}\right\rangle\left(\mathbf{u}_{, k} \cdot \mathbf{u}_{, l}\right)\right) \delta D\right. \\
\left.-\frac{D}{2}\left(\mathbf{u}_{, k} \cdot \mathbf{u}_{, l}\right) \delta\left\langle\xi^{k} \xi^{l}\right\rangle+(D-1) \delta p\right]
\end{gathered}
$$


where evenness of the generalized Helmholtz operator under integration by parts is used in obtaining the first term. Finally, the Lie-Poisson bracket defined on the dual of the algebra in equation (7.1) is given explicitly by

$$
\begin{aligned}
\{F, H\}\left(\mathbf{m}, D,\left\langle\xi^{k} \xi^{l}\right\rangle\right) & \\
=-\int d^{3} x & \left\{\frac{\delta F}{\delta m_{i}}\left(\partial_{j} m_{i}+m_{j} \partial_{i}\right) \frac{\delta H}{\delta m_{j}}+\frac{\delta F}{\delta m_{i}}\left(D \partial_{i}\right) \frac{\delta H}{\delta D}+\frac{\delta F}{\delta D}\left(\partial_{j} D\right) \frac{\delta H}{\delta m_{j}}\right. \\
& -\frac{\delta F}{\delta m_{i}}\left(\left\langle\xi^{c} \xi^{d}\right\rangle_{, i}+\partial_{a}\left\langle\xi^{a} \xi^{d}\right\rangle \delta_{i}^{c}+\partial_{a}\left\langle\xi^{c} \xi^{a}\right\rangle \delta_{i}^{d}\right) \frac{\delta H}{\delta\left\langle\xi^{c} \xi^{d}\right\rangle} \\
& \left.-\frac{\delta F}{\delta\left\langle\xi^{k} \xi^{l}\right\rangle}\left(-\left\langle\xi^{k} \xi^{l}\right\rangle_{, j}+\left\langle\xi^{a} \xi^{l}\right\rangle \partial_{a} \delta_{j}^{k}+\left\langle\xi^{k} \xi^{a}\right\rangle \partial_{a} \delta_{j}^{l}\right) \frac{\delta H}{\delta m_{j}}\right\} .
\end{aligned}
$$

In the second line of this Lie-Poisson bracket we see the contributions of the fluctuation covariance to the motion equation, cf. (6.7), and in the third line we see the operator in the covariance dynamics itself, cf. (6.6).

The dynamical system consisting of the motion equation (6.9), the continuity equation (2.7) and the Lagrangian mean covariance equation (6.6) now emerges as a Lie-Poisson Hamiltonian system in the form

$$
\frac{\partial \mu}{\partial t}=\{\mu, H\}, \quad \text { with } \quad \mu \in\left(\mathbf{m}, D,\left\langle\xi^{k} \xi^{l}\right\rangle\right),
$$

with Hamiltonian $H$ given in equation (7.3) and implying conservation of the following energy, cf. equation (3.60) and equation (6.38) with $\left.\mathbf{m}\right|_{D=1}=\mathbf{v}$,

$$
E=\frac{1}{2} \int d^{3} x\left[\mathbf{v} \cdot\left(1-\partial_{k}\left\langle\xi^{k} \xi^{l}\right\rangle \partial_{l}\right)^{-1} \mathbf{v}\right]=\frac{1}{2} \int d^{3} x\left[|\mathbf{u}|^{2}+\left\langle\xi^{k} \xi^{l}\right\rangle\left(\mathbf{u}_{, k} \cdot \mathbf{u}_{, l}\right)\right] .
$$

There is a Casimir for the Lie-Poisson bracket (7.5); namely, the conserved quantity $C_{\Phi}$ in equation (6.42). This quantity satisfies the Casimir relation,

$$
\left\{C_{\Phi}, H\right\}=0, \quad \forall \Phi \text { and } \forall H .
$$

Thus, of course, $C_{\Phi}$ in equation (6.42) is also a constant of motion for the Lagrangian mean model Hamiltonian in equation (7.3).

\section{The 2D ideal LMM equations have no velocity Casimirs}

We recall the ideal Lagrangian mean equation of motion (6.17)

$$
\begin{aligned}
& \frac{\partial \mathbf{v}}{\partial t}+\mathbf{u} \cdot \boldsymbol{\nabla} \mathbf{v}=-\nabla p, \quad \boldsymbol{\nabla} \cdot \mathbf{u}=0, \\
& \text { where } \quad \mathbf{v}=\mathbf{u}-\left(\partial_{k}\left\langle\xi^{k} \xi^{l}\right\rangle \partial_{l}\right) \mathbf{u} \equiv(1-\tilde{\Delta}) \mathbf{u},
\end{aligned}
$$

The curl form (6.34) of the motion equation is

$$
\frac{\partial}{\partial t} \mathbf{v}-\mathbf{u} \times(\boldsymbol{\nabla} \times \mathbf{v})+\nabla p+u^{j} \nabla v_{j}=0 .
$$


The curl of this equation, in turn, gives the vorticity dynamics,

$$
\frac{\partial \mathbf{q}}{\partial t}+\mathbf{u} \cdot \boldsymbol{\nabla} \mathbf{q}=\mathbf{q} \cdot \nabla \mathbf{u}+\left[\boldsymbol{\nabla} v_{j} \times \nabla u^{j}\right], \quad \text { where } \quad \mathbf{q} \equiv \operatorname{curl} \mathbf{v} .
$$

In two dimensions these equations simplify because the vortex stretching term is absent, but they remain extremely nonlinear (and essentially different from the 2D Euler equations) because of the vorticity creation term. In two dimensions, we may define the stream function $\psi(\mathbf{x}, t)$ satisfying $\mathbf{u}=\hat{\boldsymbol{z}} \times \boldsymbol{\nabla} \psi$, so that,

$$
q=\hat{\boldsymbol{z}} \cdot \boldsymbol{q}=\hat{\boldsymbol{z}} \cdot \operatorname{curl}((1-\tilde{\Delta}) \hat{\boldsymbol{z}} \times \boldsymbol{\nabla} \psi)=\partial_{x}(1-\tilde{\Delta}) \psi_{x}+\partial_{y}(1-\tilde{\Delta}) \psi_{y} \equiv \mathcal{O} \psi .
$$

This equation defines the operator $\mathcal{O}$. Note that partial spatial derivatives do not commute with the dynamical Laplacian, $\tilde{\Delta}=\partial_{k}\left\langle\xi^{k} \xi^{l}\right\rangle \partial_{l}$, because of the spatial dependence in $\left\langle\xi^{k} \xi^{l}\right\rangle$.

In terms of the stream function $\psi$ and the operator $\mathcal{O}$, the $2 \mathrm{D}$ vorticity dynamics 8.4) may be written as

$$
\begin{aligned}
\frac{\partial}{\partial t} \mathcal{O} \psi+J\{\psi, \mathcal{O} \psi\}=J\left\{v_{j}, u^{j}\right\} & =-J\left\{\tilde{\Delta} \psi_{x}, \psi_{x}\right\}-J\left\{\tilde{\Delta} \psi_{y}, \psi_{y}\right\} \\
& =-\frac{1}{2} \tilde{\Delta}_{x}|\nabla \psi|_{y}^{2}+\frac{1}{2} \tilde{\Delta}_{y}|\nabla \psi|_{x}^{2},
\end{aligned}
$$

where $J\{f, g\}=f_{x} g_{y}-f_{y} g_{x}$ is the 2D Jacobi operation, subscripts denote partial derivatives and, e.g., the operator $\tilde{\Delta}_{x}=\partial_{k}\left\langle\xi^{k} \xi^{l}\right\rangle_{x} \partial_{l}$ operates to its right. The highly nonlinear right hand side of this equation prevents conservation of the domain integrated powers of the 2D Lagrangian mean vorticity, $q=\mathcal{O} \psi$. For example, the enstrophy $\int q^{2} d x d y$ is not conserved by this flow, and the Lagrangian mean vorticity $\int q d x d y$ is only conserved for certain boundary conditions (those for which $\left.\oint_{b d y} \mathbf{v} \cdot d \mathbf{u}=0\right)$. Thus, the 2D ideal LMM equations apparently have no Casimirs that depend upon the velocity. Of course, they do have the Casimir $C_{\Phi}$ in equation (6.42) associated with the determinant of the Lagrangian mean covariance.

\section{Relation to second moment turbulence closure mod- els}

By adapting the work of Chen et al. [3]- [5], we form a second moment turbulence closure model that combines the one point closure model they studied with the results of the previous sections and yields the following system of equations,

$$
\frac{\partial}{\partial t} \mathbf{v}+(\mathbf{u} \cdot \boldsymbol{\nabla}) \mathbf{v}=\nu \tilde{\Delta} \mathbf{v}-\nabla p+\mathbf{F}, \quad \boldsymbol{\nabla} \cdot \mathbf{u}=0
$$

where $\nu$ is a constant kinematic viscosity and in this case $\mathbf{v}$ is given by

$$
\mathbf{v}=\mathbf{u}-\left(\partial_{k}\left\langle\xi^{k} \xi^{l}\right\rangle \partial_{l}\right) \mathbf{u} \equiv(1-\tilde{\Delta}) \mathbf{u}
$$


Note that the Lagrangian mean fluctuation covariance appears in the dissipation operator $\tilde{\Delta}$. In the absence of the forcing $\mathbf{F}$, this viscous LMM turbulence model dissipates the energy $E$ in equation (6.38) according to

$$
\frac{d E}{d t}=-\nu \int d^{3} x\left[\operatorname{tr}\left(\boldsymbol{\nabla} \mathbf{u}^{T} \cdot\langle\boldsymbol{\xi} \boldsymbol{\xi}\rangle \cdot \boldsymbol{\nabla} \mathbf{u}\right)+\tilde{\Delta} \mathbf{u} \cdot \tilde{\Delta} \mathbf{u}\right]
$$

This strictly negative energy dissipation law is the reason for adding viscosity with $\tilde{\Delta}$, instead of using the ordinary Laplacian operator.

These equations are closed by the dissipative dynamical equation (4.36) for the advected Lagrangian mean covariance, rewritten as,

$$
\frac{d}{d t}\left\langle\xi^{k} \xi^{l}\right\rangle=u_{, j}^{k}\left\langle\xi^{j} \xi^{l}\right\rangle+\left\langle\xi^{k} \xi^{j}\right\rangle u_{, j}^{l}-\frac{1}{\tau}\left(\left\langle\xi^{k} \xi^{l}\right\rangle-\alpha^{2} \delta^{k l}\right)+\lambda \frac{\alpha^{2}}{\tau} \tilde{\Delta}\left\langle\xi^{k} \xi^{l}\right\rangle .
$$

The boundary conditions for this dissipative $\mathbf{L M} \boldsymbol{M}$ model for a fixed boundary are $\mathbf{v}=0, \mathbf{u}=0$, for the velocities and either $\langle\boldsymbol{\xi} \boldsymbol{\xi}\rangle \cdot \hat{\boldsymbol{n}}=0$ when dissipation is absent in the dynamics of $\langle\boldsymbol{\xi} \xi\rangle$, or equation (9.4) without the diffusion term when such dissipation is present.

As mentioned before, isotropy and homogeneity of the Lagrangian mean covariance tensor $\left\langle\xi^{k} \xi^{l}\right\rangle$ are not preserved under the dynamics of equation (9.4) for nontrivial velocity shear. Therefore, the VCHE or NS- $\alpha$ closure model of Chen et al. [3] - [5], is not an invariant subsystem of the dissipative LMM model in equations (9.1)-(9.4). However, the phenomenological addition of relaxation and dissipation to the covariance dynamics does make the dissipative LMM model relax to the VCHE or NS- $\alpha$ closure model, whose validity in steady state has been verified by comparison with experimental data, [3]- [5]. These dissipative LMM equations comprise a 3D dynamically self-consistent second-moment turbulence closure model, whose solution may be sought as a dynamical systems problem, e.g., as an initial value problem for decaying turbulence, or as motion of a forced dissipative system.

The symmetric quantity $\boldsymbol{\kappa}_{S}=d\langle\boldsymbol{\xi} \boldsymbol{\xi}\rangle / d t$ in equation (9.4) is called the Taylor diffusivity [18] and is sometimes used in turbulence modeling as a semi-empirical model for passive scalar diffusion [17], [19], [40]. This application will be considered further in Section 10, when we include the effects of rotation and stratification, as well. For now, the main effect of the fluctuations in equations (9.1) - (9.2) from the viewpoint of the Navier-Stokes equations for an incompressible fluid is to smooth the transport velocity $\mathbf{u}$ relative to the circulation velocity $\mathbf{v}$, by inversion of the Helmholtz operator $(1-\tilde{\Delta})$. This smoothing tends to suppress triad interactions at wave numbers greater in magnitude than about $|\langle\xi \xi\rangle|^{-1 / 2}$. Therefore, the cascade of the spectral kinetic energy density $\hat{E}(\mathbf{k})=\frac{1}{2}(\widehat{\mathbf{u} \cdot \mathbf{v}})(\mathbf{k})$ as a function of wave number will be suppressed for $|\mathbf{k}|>|\langle\boldsymbol{\xi} \xi\rangle|^{-1 / 2}$. Hence, viscous dissipation should take over at length scales smaller than the local length scale given by $|\langle\boldsymbol{\xi} \xi\rangle|^{1 / 2}(\mathbf{x}, t)$ and suppress the effects of nonlinearity at these smaller scales, just as if the LMM model in (9.1) - (9.4) were an adaptive LES scheme. The LES aspects of the dissipative LMM model will be pursued elsewhere. 


\section{Geophysical applications - adding rotation and strat- ification to the Lagrangian mean model}

\subsection{Formulation of Lagrangian mean Euler-Boussinesq (LMEB) equations}

At leading order in $|\boldsymbol{\xi}|^{2}$, introduction of rotation and stratification alters the averaged approximate Lagrangian $\langle L\rangle$ in equation (3.34) to the following form,

$$
\langle L\rangle=\int d^{3} x\left\{\frac{D}{2}\left[|\mathbf{u}|^{2}+\left\langle\xi^{k} \xi^{l}\right\rangle\left(\mathbf{u}_{, k} \cdot \mathbf{u}_{, l}\right)\right]+D \mathbf{R}(\mathbf{x}) \cdot \mathbf{u}-g b D z+p[1-D]\right\} .
$$

The corresponding Euler-Poincaré equations are, cf. [1], [2],

$$
\begin{aligned}
& \frac{d}{d t} \mathbf{v}-\mathbf{u} \times \operatorname{curl} \mathbf{R}(\mathbf{x})+\nabla p+g b \hat{\mathbf{z}}=0, \quad \text { with } \quad \boldsymbol{\nabla} \cdot \mathbf{u}=0, \\
& \text { where } \frac{d}{d t} \equiv\left(\frac{\partial}{\partial t}+\mathbf{u} \cdot \boldsymbol{\nabla}\right), \quad \mathbf{v} \equiv(1-\tilde{\Delta}) \mathbf{u}, \quad \tilde{\Delta} \equiv \boldsymbol{\nabla} \cdot\langle\boldsymbol{\xi} \xi\rangle \cdot \nabla, \\
& \frac{d b}{d t}=0, \quad \text { and } \frac{d}{d t}\langle\boldsymbol{\xi} \boldsymbol{\nabla}\rangle=\langle\boldsymbol{\xi} \boldsymbol{\xi}\rangle \cdot \nabla \mathbf{u}+\nabla \mathbf{u}^{\mathrm{T}} \cdot\langle\boldsymbol{\xi} \boldsymbol{\xi}\rangle,
\end{aligned}
$$

with boundary conditions,

$$
\mathbf{v} \cdot \hat{\boldsymbol{n}}=0 \quad \mathbf{u}=0, \quad \text { and } \quad\langle\boldsymbol{\xi} \boldsymbol{\xi}\rangle \cdot \hat{\boldsymbol{n}}=0 \quad \text { on a fixed boundary }
$$

These Lagrangian mean Euler-Boussinesq (LMEB) equations describe the Lagrangian mean effects of fluctuations on the ideal motion of a stratified incompressible fluid in a rotating reference frame, given in the Eulerian description. (Note that the $\langle\boldsymbol{\xi} \xi\rangle$ equation in (10.3) does not refer to internal waves. See [14] for a discussion of the Lagrangian mean effects of internal waves from a viewpoint similar to the one taken here.) The motion equation for this system may be rewritten as

$$
\frac{\partial}{\partial t} \mathbf{v}-\mathbf{u} \times \operatorname{curl}(\mathbf{v}+\mathbf{R})+u^{j} \boldsymbol{\nabla} v_{j}+\nabla p+g b \boldsymbol{\nabla} z=0 .
$$

From this expression, we immediately find the Kelvin-Noether circulation theorem showing how the covariance of the fluctuations interacts with the buoyancy to generate mean total circulation. Namely,

$$
\frac{d}{d t} \oint_{\gamma(\mathbf{u})}(\mathbf{v}+\mathbf{R}) \cdot d \mathbf{x}=\iint_{S(\mathbf{u})}\left[\nabla v_{j} \times \nabla u^{j}-g \boldsymbol{\nabla} b \times \nabla z\right] \cdot d \mathbf{S}
$$

where the closed curve $\gamma(\mathbf{u})$ moves with the Lagrangian mean fluid velocity $\mathbf{u}$ and is the boundary of the surface $S(\mathbf{u})$. The terms $\left[\boldsymbol{\nabla} v_{j} \times \boldsymbol{\nabla} u^{j}\right]$ and $[g \boldsymbol{\nabla} b \times \boldsymbol{\nabla} z]$ are both vorticity creation terms in this Lagrangian mean Kelvin-Noether circulation theorem. Therefore, the fluctuations act together with buoyancy to create circulation of the sum $\mathbf{v}+\mathbf{R}$ around closed fluid loops moving with velocity $\mathbf{u}$. In 
particular, the vorticity creation term $\left[\nabla v_{j} \times \nabla u^{j}\right]$ may cause mixing across isopycnal (constant $b$ ) surfaces, even when the buoyancy is stably stratified. According to the interpretation given in Section 1 , this is the effect of the Stokes mean drift velocity.

The fluctuations and the buoyancy also act together in the conserved energy for the LMEB system (10.2) - (10.4). Namely, cf. equation (1.11),

$$
E_{L M E B}=\int d^{3} x\left[\frac{1}{2}\left(|\mathbf{u}|^{2}+\operatorname{tr}\left(\boldsymbol{\nabla} \mathbf{u}^{\mathrm{T}} \cdot\langle\boldsymbol{\xi} \boldsymbol{\xi}\rangle \cdot \boldsymbol{\nabla u}\right)\right)+g b z\right] .
$$

This expression shows the trade offs in energetics among translational kinetic energy, Lagrangian mean velocity shear combined with the fluctuations, and gravitational potential energy that may occur in the dynamics of the LMEB model. These energetic tradeoffs should have implications for the stability properties of the LMEB model's equilibrium solutions. We note that the LMEB model has all the same equilibrium solutions of the original Euler-Boussinesq equations (namely, those on the invariant manifold $\langle\boldsymbol{\xi} \boldsymbol{\xi}\rangle=0$ ) in addition to others associated with critical points of the sum of the energy $E_{L M E B}$ and the conserved Casimirs for the EAB theory,

$$
C_{L M E B}=\int d^{3} x D \Phi\left(b, \tilde{\Delta} b, D^{2} \operatorname{det}\langle\xi \xi\rangle\right) \text { for an arbitrary function, } \Phi .
$$

These quantities Poisson commute with every Hamiltonian $H$ : that is, under a modification of the Lie-Poisson bracket in equation (7.5) to include the buoyancy variable $b$, as follows,

$$
\{F, H\}_{L M E B}=\{F, H\} \text { 7.5 }-\int d^{3} x\left[\frac{\delta F}{\delta m_{i}}\left(-b_{, i}\right) \frac{\delta H}{\delta b}+\frac{\delta F}{\delta b}\left(b_{, j}\right) \frac{\delta H}{\delta m_{j}}\right],
$$

we have $\left\{C_{L M E B}, H\right\}_{L M E B}=0$, for all $H[\mathbf{m}, D,\langle\boldsymbol{\xi} \boldsymbol{\xi}\rangle, b]$.

\subsection{Passive scalar diffusion in the LMEB model.}

Following Bennett [19], we introduce the symmetric Taylor diffusivity tensor [18] as

$$
\kappa_{S}^{k l} \equiv \frac{1}{2} \frac{d}{d t}\left\langle\xi^{k} \xi^{l}\right\rangle, \quad \text { or } \quad \boldsymbol{\kappa}_{S} \equiv \frac{1}{2} \frac{d}{d t}\langle\boldsymbol{\xi} \boldsymbol{\xi}\rangle
$$

According to the semi-empirical theory [17], [19], [40], the corresponding passive scalar equation with this diffusivity is

$$
\frac{d b}{d t}=\nabla \cdot\left(\kappa_{S} \cdot \nabla b\right)
$$

As indicated here, we shall adopt this equation for the dissipative dynamics of the buoyancy, even though $b$ is not strictly passive. The present theory provides a dynamical equation (10.3) for the evolution of the Taylor diffusivity tensor, $\boldsymbol{\kappa}_{S}$. Thus, passive scalars in this theory advect and diffuse according to equation (10.11) with tensor diffusivity given by equation (6.40) as

$$
2 \boldsymbol{\kappa}_{S} \equiv \frac{d}{d t}\langle\boldsymbol{\xi} \boldsymbol{\xi}\rangle=\langle\boldsymbol{\xi} \boldsymbol{\xi}\rangle \cdot \nabla \mathbf{u}+\nabla \mathbf{u}^{\mathrm{T}} \cdot\langle\boldsymbol{\xi} \boldsymbol{\xi}\rangle
$$


Hence, the passive scalar equation we find is,

$$
\frac{d b}{d t}=\frac{1}{2}\left(\boldsymbol{\nabla} \cdot\left(\langle\boldsymbol{\xi} \boldsymbol{\xi}\rangle \cdot \nabla \mathbf{u}+\nabla \mathbf{u}^{\mathrm{T}} \cdot\langle\boldsymbol{\xi} \boldsymbol{\xi}\rangle\right) \cdot \boldsymbol{\nabla}\right) b,
$$

where the Lagrangian mean covariance $\langle\boldsymbol{\xi} \xi\rangle$ and the velocity shear tensor $\boldsymbol{\nabla u}$ are determined self-consistently from the LMEB dynamics, suitably modified to include dissipation. We see that the tensor diffusivity $\boldsymbol{\kappa}_{S}=\frac{1}{2}\left(\langle\boldsymbol{\xi} \boldsymbol{\xi}\rangle \cdot \boldsymbol{\nabla u}+\boldsymbol{\nabla} \mathbf{u}^{\mathrm{T}} \cdot\langle\boldsymbol{\xi} \boldsymbol{\xi}\rangle\right)$ is determined dynamically as the symmetrized product of the Lagrangian mean covariance and the mean velocity shear of the flow. As we have discussed, equation (10.12) implies that the covariance $\langle\boldsymbol{\xi} \xi\rangle$ at any time will be increasing along the instantaneous positive eigendirections of the velocity shear tensor $\boldsymbol{\nabla} \mathbf{u}$. Conservation of energy (10.7) should control this tendency, in general, because the cost in energy for the covariance to grow is highest precisely where the growth rate is highest.

The covariance dynamics following from the Taylor hypothesis for the advection of turbulent structures by the (Lagrangian) mean flow implies the determinant of $\langle\boldsymbol{\xi} \boldsymbol{\xi}\rangle$ will be conserved along flow lines in the incompressible case. Therefore, the covariance will remain finite and nonzero. However, if any additional dissipation is needed to moderate the growth of $\langle\boldsymbol{\xi} \xi\rangle$ due to shear forcing, equation (9.4) may be used as an alternative expression for the Taylor diffusivity.

Finally, following Chen et al. [3]- [5], we introduce viscosity into the motion equation for the Lagrangian mean Euler-Boussinesq theory as $\tilde{\Delta}$-diffusion of momentum $\mathbf{v}$, cf. equation (9.1), with boundary conditions as discussed after equation (9.4). This final step - the semi-empirical introduction of dissipation - completes our formulation of the LMEB equation set (1.1) - (1.6) for the Lagrangian mean motion of a rotating stratified incompressible fluid. In what follows, we shall consider some lower dimensional subcases of this Lagrangian mean motion in Section 11, then turn our attention to the corresponding Eulerian mean theory in Sections 12 and 13 .

Remark on asymmetry of the diffusivity and other modeling steps. Because of the Earth's rotation, asymmetric diffusivity may be expected in the ocean. This is an additional modeling step, which will be pursued elsewhere. For discussions of this aspect, see, e.g., the review by Davis [41] and references therein. Other modeling steps would address the creation of fluctuation covariance, as well as its dissipation, or treat the covariance between fluctuations at two different times. We do not pursue these other sub-gridscale modeling directions here. Instead, we proceed from the Taylor hypothesis (3.17) that the Lagrangian mean fluctuation covariance is advected by the Lagrangian mean fluid velocity.

\section{Lagrangian mean motion in fewer dimensions}

\subsection{D Lagrangian mean rotating shallow water (LMRSW) equa- tions}

To the averaged approximated LMEB Lagrangian $\langle L\rangle$ in equation (10.1) we apply the standard shallow water approximations: neglect vertical gradients; neglect 
kinetic energy of vertical motion; assume constant density; and integrate in the vertical direction from the bottom topography at $z=-B(x, y)$ to the free surface at $z=h(x, y, t)$. These approximations lead to a new Lagrangian $\langle L\rangle_{R S W}^{L}$ for Lagrangian mean rotating shallow water (LMRSW) dynamics,

$$
\begin{aligned}
\langle L\rangle_{R S W}^{L}=\int d x d y\{ & \frac{D}{2}\left[|\mathbf{u}|^{2}+\left\langle\xi^{k} \xi^{l}\right\rangle\left(\mathbf{u}_{, k} \cdot \mathbf{u}_{, l}\right)\right] \\
& \left.+D \mathbf{R}(\mathbf{x}) \cdot \mathbf{u}-\frac{1}{2} g D^{2}+g D B(x, y)\right\} .
\end{aligned}
$$

Here $D=h+B(x, y)$ is the total depth of the water. The corresponding EulerPoincaré motion equation for LMRSW dynamics in momentum conservation form is, cf. equations (6.12) - (6.14),

$$
\frac{\partial m_{i}}{\partial t}=-\frac{\partial}{\partial x^{j}}\left(m_{i} u^{j}+\frac{1}{2} g D^{2} \delta_{i}^{j}\right)+g D B_{, i}(x, y)+D u_{j} R_{, i}^{j},
$$

where one defines momentum density components $m_{i}$, for $i=1,2$ by

$$
m_{i} \equiv \frac{\delta\left\langle L_{S W}\right\rangle}{\delta u^{i}}=D\left(u_{i}+R_{i}\right)-\left(D\left\langle\xi^{k} \xi^{l}\right\rangle u_{i, l}\right)_{, k} \equiv D\left(1-\tilde{\Delta}_{D}\right) u_{i}+D R_{i} .
$$

The depth $D$ satisfies the continuity equation,

$$
\frac{\partial D}{\partial t}+\nabla \cdot(D \mathbf{u})=0
$$

and $\langle\xi \xi\rangle$ satisfies the Lagrangian mean covariance dynamics inherited from the Taylor hypothesis (3.17),

$$
\frac{d}{d t}\langle\boldsymbol{\xi} \boldsymbol{\xi}\rangle=\langle\xi \xi\rangle \cdot \nabla \mathbf{u}+\nabla \mathbf{u}^{\mathrm{T}} \cdot\langle\boldsymbol{\xi} \boldsymbol{\xi}\rangle
$$

In terms of the circulation velocity $\mathbf{v}=\left(1-\tilde{\Delta}_{D}\right) \mathbf{u}$ defined as before, but now in $2 \mathrm{D}$, we find the motion equation for $\mathbf{v}$,

$$
\left(\frac{\partial}{\partial t}+\mathbf{u} \cdot \boldsymbol{\nabla}\right) \mathbf{v}-\mathbf{u} \times \operatorname{curl} \mathbf{R}(\mathbf{x})+g \boldsymbol{\nabla}(D-B(x, y))=0 .
$$

This is the same as the standard motion equation for rotating ideal shallow water dynamics, modulo the substitution $\mathbf{u} \rightarrow \mathbf{v}$ and the additional dynamics for the covariance $\langle\boldsymbol{\xi} \boldsymbol{\xi}\rangle$. This motion equation for LMRSW implies the Kelvin-Noether circulation theorem, cf. equation (6.32),

$$
\frac{d}{d t} \oint_{\gamma(\mathbf{u})}(\mathbf{v}+\mathbf{R}) \cdot d \mathbf{x}=\iint_{S(\mathbf{u})} d v_{j} \wedge d u^{j}=\iint_{S(\mathbf{u})}\left[\nabla v_{j} \times \nabla u^{j}\right] \cdot d \mathbf{S} .
$$

Thus, as for the LMM model, the fluctuations have the effect of generating (total) circulation. The curl of the LMRSW motion equation yields, with $q=\hat{z} \cdot \operatorname{curl}(\mathbf{v}+\mathbf{R})$,

$$
\left(\frac{\partial}{\partial t}+\mathbf{u} \cdot \boldsymbol{\nabla}\right)\left(\frac{q}{D}\right)=D^{-1} \hat{z} \cdot\left(\nabla v_{j} \times \nabla u^{j}\right)=D^{-1} J\left(v_{j}, u^{j}\right) .
$$


Thus, perhaps unexpectedly, the potential vorticity $q / D$ is not conserved on fluid parcels by the LMRSW model, but instead has a local creation term, $D^{-1} J\left(v_{j}, u^{j}\right)$. This creation of potential vorticity is in fact the convective mechanism by which the smoothing of velocity gradients occurs in the LMRSW model. The usual equations of rotating shallow water theory are recovered on the invariant subsystem for $\langle\xi \xi\rangle=0$, on which $\mathbf{v}=\mathbf{u}$, and the right hand side of the potential vorticity equation (11.8) vanishes.

\subsection{One dimensional Lagrangian mean shallow water (LMSW) equations}

Restricting the Lagrangian $\langle L\rangle_{R S W}^{L}$ in equation (11.1) to one dimensional motion (without rotation) results in

$$
\langle L\rangle_{S W}^{L}=\int d x\left[\frac{D}{2}\left(u^{2}+w u_{x}^{2}\right)-\frac{1}{2} g D^{2}+g D B(x)\right] .
$$

Here we denote $\langle\xi \xi\rangle$ by $w$ in one dimension. We also denote partial derivatives by subscripts and $D=h+B(x)$ is the total depth of the water. The corresponding Euler-Poincaré equations in momentum conservation form for LMSW in one dimension are, cf. equations (6.12) - (6.14),

$$
\begin{aligned}
m_{t} & =-\left(m u+\frac{1}{2} g D^{2}\right)_{x}+g D B_{x}, \\
\text { with } m \text { defined by } m & \equiv \frac{\delta\langle L\rangle_{S W}^{L}}{\delta u}=D u-\left(D w u_{x}\right)_{x}, \\
\text { where } D \text { and } w \text { satisfy } D_{t} & =-(D u)_{x}, \\
\text { and } \quad w_{t} & =-u w_{x}+2 w u_{x} .
\end{aligned}
$$

The corresponding motion equation for the momentum velocity $v=m / D$ is given by,

$$
v_{t}+u v_{x}+g(D-B(x))_{x}=0, \quad \text { where } \quad v=u-D^{-1}\left(D w u_{x}\right)_{x} .
$$

Thus, the Lagrangian mean equation in the case of one dimensional shallow water again makes just one simple change $v \rightarrow u$ in the advection term, and introduces an additional equation for the dynamics of the Lagrangian mean covariance $\langle\xi \xi\rangle=w$.

An explicit calculation using equations (11.12), (11.13) and the definition of the momentum velocity $v$ in (11.14) regains the remarkable relation (3.49), expressed in one dimension as,

$$
v_{t}+u v_{x}=\left(1-D^{-1} \partial_{x} D w \partial_{x}\right)\left(u_{t}+u u_{x}\right) .
$$

Hence, the one dimensional Lagrangian mean shallow water equation system may be rewritten as

$$
\begin{aligned}
& u_{t}+u u_{x}+g\left(1-D^{-1} \partial_{x} D w \partial_{x}\right)^{-1}(D-B)_{x}=0 \\
& D_{t}+u D_{x}+D u_{x}=0 \\
& w_{t}+u w_{x}-2 w u_{x}=0
\end{aligned}
$$


This system for $w \neq 0$ is no longer hyperbolic; rather, it is conservative and dispersive. However, when $w=0$, we again recover the usual shallow water dynamics as an invariant subsystem.

One may also obtain the equations for Lagrangian mean polytropic gas dynamics with pressure-density relation $p=p_{0}\left(D / D_{0}\right)^{\gamma}$, by replacing the motion equation in the Lagrangian mean shallow water system (11.16) - (11.18) with

$$
u_{t}+u u_{x}+\frac{p_{0} \gamma}{D_{0}^{\gamma}}\left(1-D^{-1} \partial_{x} D w \partial_{x}\right)^{-1} D^{\gamma-2} D_{x}=0 .
$$

Thus, equations (11.17) - 11.19) provide a system of Lagrangian mean polytropic gas equations, which is no longer hyperbolic for $w \neq 0$, but which recovers the usual polytropic gas dynamics as an invariant subsystem when $w=0$. This system conserves the energy,

$$
E=\int d x\left[\frac{D}{2}\left(u^{2}+w u_{x}^{2}\right)+\frac{p_{o}}{\gamma-1} \frac{D^{\gamma}}{D_{0}^{\gamma}}\right]
$$

The one dimensional Lagrangian mean models for shallow water and polytropic gas dynamics given in this Section should provide ample opportunities for testing the dynamical effects of fluctuations on one dimensional Lagrangian mean fluid motion, without requiring the more intensive numerics and analysis needed in higher dimensions. (The three dimensional version of the Lagrangian mean polytropic gas equations is readily obtained at this point.)

\subsection{The Lagrangian mean Riemann (LMR) equation}

The process we apply here of fast-slow decomposition, followed by Taylor approximation and averaging at fixed Lagrangian fluid label in Hamilton's principle for ideal fluids produces the following situation. There are more equations in the averaged approximate model than in the original model and they include the original model as an invariant subsystem in which the fluctuation covariance vanishes.

The simplest situation for which this occurs is probably the Riemann equation,

$$
u_{t}+3 u u_{x}=0
$$

which describes one dimensional shock formation and is the Euler-Poincaré equation for the Lagrangian,

$$
L_{R}=\int d x \frac{1}{2} u^{2}
$$

The corresponding averaged approximate Lagrangian for this problem is (again writing $\langle\xi \xi\rangle=w$ for the covariance in one dimension)

$$
\langle L\rangle_{R}=\int d x \frac{1}{2}\left(u^{2}+w u_{x}^{2}\right)
$$


whose Euler-Poincaré equation is

$$
v_{t}+\left(\frac{1}{2} u^{2}+\frac{1}{2} w u_{x}^{2}+u v\right)_{x}=0,
$$

with

$$
v=\frac{\delta\langle L\rangle_{R}}{\delta u}=u-\left(w u_{x}\right)_{x},
$$

and the one dimensional Lagrangian mean covariance $w$ satisfies

$$
w_{t}=-u w_{x}+2 w u_{x} .
$$

The Lagrangian mean Riemann system $(11.24)$ - $(11.26)$ can be rewritten equivalently in (nonlocal) characteristic form as

$$
\begin{aligned}
u_{t}+u u_{x} & =-\left(1-\partial_{x} w \partial_{x}\right)^{-1} \partial_{x}\left(u^{2}-\frac{1}{2} w u_{x}^{2}\right), \\
\text { and } w_{t} & =-u w_{x}+2 w u_{x} .
\end{aligned}
$$

We compare the Lagrangian mean Riemann system $(\sqrt{11.24})-(\sqrt{11.26})$ with the Camassa-Holm $(\mathrm{CH})$ equation, a one dimensional completely integrable nonlinear dispersive shallow water model [21], 22],

$$
\left(u-u_{x x}\right)_{t}+\left(\frac{1}{2} u^{2}-\frac{1}{2} u_{x}^{2}+u\left(u-u_{x x}\right)\right)_{x}=0,
$$

which may also be written in (nonlocal) characteristic form closely similar to equation $(11.27)$ as

$$
u_{t}+u u_{x}=-\left(1-\partial_{x}^{2}\right)^{-1} \partial_{x}\left(u^{2}+\frac{1}{2} u_{x}^{2}\right) .
$$

The Camassa-Holm equation (11.29) is the Euler-Poincaré equation for the Lagrangian

$$
L_{C H}=\int d x \frac{1}{2}\left(u^{2}+u_{x}^{2}\right) .
$$

Further discussions of the solution behavior for the Lagrangian mean Riemann system $(11.24)-(11.26)$, including its traveling waves (which may be solved explicitly) will be given elsewhere [42]. For now, we simply observe that there is a key difference in sign between the LMR equation (11.27) and the $\mathrm{CH}$ equation (11.30).

\section{Eulerian mean theory of advected fluctuations}

Here we develop an alternative Euler-Poincaré theory of advected fluctuations from the viewpoint of Eulerian averaging. We have the same point of departure as for the Lagrangian mean theory, namely the Lagrangian (2.10) in the Eulerian description,

$$
L(\omega)=\int d^{3} x\left\{\frac{D}{2}|\mathbf{U}(\mathbf{x}, t ; \omega)|^{2}+P(\mathbf{x}, t ; \omega)(1-D(\mathbf{x}, t ; \omega))\right\} .
$$


The traditional Reynolds decomposition of fluid velocity is expressed at a given position $\mathrm{x}$ in terms of the Eulerian mean fluid velocity, $\overline{\mathrm{U}}$ as

$$
\mathbf{U}(\mathbf{x}, t ; \omega) \equiv \overline{\mathbf{U}}(\mathbf{x}, t)+\mathbf{U}^{\prime}(\mathbf{x}, t ; \omega) .
$$

According to equation (4.6) the Eulerian velocity fluctuation $\mathbf{U}^{\prime}(\mathbf{x}, t ; \omega)$ is related to the Eulerian displacement fluctuation — denoted as $\boldsymbol{\zeta}(\mathbf{x}, t ; \omega)$ - by

$$
\frac{\partial \zeta}{\partial t}+\overline{\mathbf{U}} \cdot \nabla \boldsymbol{\zeta}=\boldsymbol{\zeta} \cdot \nabla \overline{\mathbf{U}}+\mathbf{U}^{\prime}(\mathbf{x}, t ; \omega) .
$$

As discussed in Section 4, for purely Eulerian velocity fluctuations as in equation (12.2), this relation separates into two relations: the "Taylor-like" hypothesis of [23],

$$
\frac{\partial \zeta}{\partial t}+\overline{\mathbf{U}} \cdot \nabla \boldsymbol{\zeta}=0
$$

and the relation

$$
0=\boldsymbol{\zeta} \cdot \nabla \overline{\mathbf{U}}+\mathbf{U}^{\prime}(\mathbf{x}, t ; \omega) .
$$

Hence, the Reynolds velocity decomposition (12.2) separates the Lagrangian (12.1) into its mean and fluctuating pieces, as

$$
L(\omega)=\int d^{3} x\left\{\frac{D}{2}\left|\overline{\mathbf{U}}(\mathbf{x}, t)+\mathbf{U}^{\prime}\right|^{2}+P(\mathbf{x}, t)(1-D(\mathbf{x}, t))\right\} .
$$

No modification is needed in the pressure constraint in this Lagrangian, because the Eulerian mean preserves the condition that the velocity be divergenceless; hence, $\boldsymbol{\nabla} \cdot \overline{\mathbf{U}}=0$. It remains only to take the Eulerian mean of this Lagrangian, in which we assume $\langle\boldsymbol{\zeta}\rangle^{E}=0$. The Eulerian mean averaging process at fixed position $\mathbf{x}$ is denoted $\langle\cdot\rangle^{E}$ with, e.g.,

$$
\overline{\mathbf{U}}(\mathbf{x}, t)=\langle\mathbf{U}(\mathbf{x}, t ; \omega)\rangle^{E} \equiv \lim _{T \rightarrow \infty} \frac{1}{T} \int_{-T}^{T} \mathbf{U}(\mathbf{x}, t ; \omega) d \omega .
$$

By equation (12.5), the Eulerian mean kinetic energy due to the velocity fluctuation satisfies

$$
\left\langle\left|\mathbf{U}^{\prime}\right|^{2}\right\rangle^{E}=\left\langle\zeta^{k} \zeta^{l}\right\rangle^{E} \overline{\mathbf{U}}_{, k} \cdot \overline{\mathbf{U}}_{, l} .
$$

Thus, we find, the following Eulerian mean Lagrangian, (cf. equation (3.54) for the corresponding Lagrangian mean form)

$$
\langle L\rangle^{E}=\int d^{3} x\left\{\frac{D}{2}\left[|\overline{\mathbf{U}}(\mathbf{x}, t)|^{2}+\left\langle\zeta^{k} \zeta^{l}\right\rangle^{E} \overline{\mathbf{U}}_{, k} \cdot \overline{\mathbf{U}}_{, l}\right]+P(\mathbf{x}, t)(1-D(\mathbf{x}, t))\right\} .
$$

The advection relation (12.4) implies a similar advection of each component of the symmetric Eulerian mean covariance tensor $\left\langle\zeta^{k} \zeta^{l}\right\rangle^{E}$. Thus, we have

$$
\left(\frac{\partial}{\partial t}+\overline{\mathbf{U}} \cdot \nabla\right)\left\langle\zeta^{k} \zeta^{l}\right\rangle^{E}=0 .
$$


This relation the continuity equation for the volume element $D$,

$$
\frac{\partial D}{\partial t}+\nabla \cdot D \overline{\mathbf{U}}=0
$$

complete the auxiliary equations needed for deriving the equation of motion for the Eulerian mean velocity $\overline{\mathbf{U}}$ from the averaged Lagrangian $\langle L\rangle^{E}$ in $(12.9)$ by using the Euler-Poincaré theory.

The results of Holm, Marsden and Ratiu [1], allow one to compute the EulerPoincaré equation for the Lagrangian $\langle L\rangle^{E}\left(\overline{\mathbf{U}}, D,\left\langle\zeta^{k} \zeta^{l}\right\rangle^{E}\right)$ in $(12.9)$ depending on the Eulerian mean velocity $\overline{\mathbf{U}}$, and advected quantities $D$ and $\left.\left\langle\zeta^{k}\right\rangle^{t}\right\rangle^{E}$ as, cf. equations (2.11) and (6.7),

$$
\begin{aligned}
0=\left(\frac{\partial}{\partial t}+\right. & \left.\bar{U}^{j} \frac{\partial}{\partial x^{j}}\right) \frac{1}{D} \frac{\delta\langle L\rangle^{E}}{\delta \bar{U}^{i}}+\frac{1}{D} \frac{\delta\langle L\rangle^{E}}{\delta \bar{U}^{j}} \bar{U}_{, i}^{j} \\
& -\frac{\partial}{\partial x^{i}} \frac{\delta\langle L\rangle^{E}}{\delta D}+\frac{1}{D} \frac{\delta\langle L\rangle^{E}}{\delta\left\langle\zeta^{k} \zeta^{l}\right\rangle^{E}} \frac{\partial}{\partial x^{i}}\left\langle\zeta^{k} \zeta^{l}\right\rangle^{E}
\end{aligned}
$$

Thus, the Euterian mean covariance $(\zeta \zeta)^{E}$ satisfying the scalar advection relation (12.10) contributes considerably simpler reactive forces than those arising in equation (6.7) from the Lagrangian mean covariance satisfying the tensor advection equation (6.5). We compute the following variational derivatives of the averaged approximate Lagrangian $\langle L\rangle^{E}$ in equation (12.9)

$$
\begin{aligned}
\frac{1}{D} \frac{\delta\langle L\rangle^{E}}{\delta \overline{\mathbf{U}}} & =\overline{\mathbf{U}}-\frac{1}{D}\left(\partial_{k} D\left\langle\zeta^{k} \zeta^{l}\right\rangle^{E} \partial_{l}\right) \overline{\mathbf{U}} \equiv \mathbf{V} \\
\frac{\delta\langle L\rangle^{E}}{\delta D} & =-P+\frac{1}{2}|\overline{\mathbf{U}}|^{2}+\frac{1}{2}\left\langle\zeta^{k} \zeta^{l}\right\rangle^{E}\left(\overline{\mathbf{U}}_{, k} \cdot \overline{\mathbf{U}}_{, l}\right) \equiv-P_{t o t}^{E} \\
\frac{\delta\langle L\rangle^{E}}{\delta P} & =1-D, \\
\frac{\delta\langle L\rangle^{E}}{\delta\left\langle\zeta^{k} \zeta^{l}\right\rangle^{E}} & =\frac{D}{2}\left(\overline{\mathbf{U}}_{, k} \cdot \overline{\mathbf{U}}_{, l}\right) .
\end{aligned}
$$

Of course, these variational derivatives are in the same form as in equation set (6.8). However, the variables here are Eulerian mean quantities and they will enter a different Euler-Poincaré equation, namely, (12.12), instead of (6.7). This difference arises because the Eulerian mean covariance $\left\langle\zeta^{k} \zeta^{l}\right\rangle^{E}$ advects as an array of scalars under the Eulerian mean evolution, rather than as the components of a symmetric tensor. Consequently, the Euler-Poincaré equation $(12.12)$ for this averaged Lagrangian takes the form,

$$
\frac{\partial V_{i}}{\partial t}+\bar{U}^{j} V_{i, j}+V_{j} \bar{U}_{, i}^{j}=-\frac{\partial P_{t o t}^{E}}{\partial x^{i}}-\frac{1}{2}\left(\overline{\mathbf{U}}_{, k} \cdot \overline{\mathbf{U}}_{, l}\right)\left\langle\zeta^{k} \zeta^{l}\right\rangle_{, i}^{E},
$$

where $\quad V_{i}=\bar{U}_{i}-\tilde{\Delta}_{D}^{E} \bar{U}_{i} \quad$ with $\quad \tilde{\Delta}_{D}^{E} \equiv \frac{1}{D}\left(\partial_{k} D\left\langle\zeta^{k} \zeta^{l}\right\rangle^{E} \partial_{l}\right) \quad$ and $\quad \bar{U}_{, i}^{i}=0$.

The boundary conditions we shall choose for this motion equation are

$$
\mathbf{V} \cdot \hat{\boldsymbol{n}}=0, \quad \overline{\mathbf{U}}=0, \quad \text { and } \quad \hat{\boldsymbol{n}} \cdot\langle\boldsymbol{\xi} \boldsymbol{\xi}\rangle^{E}=0, \quad \text { on a fixed boundary }
$$


Then, provided the Helmholtz operator $1-\tilde{\Delta}_{D}^{E}$ for $D=1$ may be inverted, the Eulerian mean pressure $P$ may be obtained by solving an elliptic equation.

Contrasting the Euler-Poincaré equation (12.14) with the $\mathrm{CH}$ equation. When the Eulerian mean covariance is isotropic and homogeneous, so that $\left\langle\zeta^{k} \zeta^{l}\right\rangle^{E}=$ $\alpha^{2} \delta^{k l}$, for a constant length scale $\alpha$, then this equation reduces to the $n$-dimensional Camassa-Holm equation introduced in [1], [2]. Thus, the $n$-dimensional $\mathrm{CH}$ equation set is an invariant subsystem of the Euler-Poincare system (12.14), with definition (12.15) and advection law (12.10), because the initial condition $\left\langle\zeta^{k} \zeta^{l}\right\rangle^{E}=\alpha^{2} \delta^{k l}$ is invariant under the dynamics of equation (12.10).

Physical interpretation of $\mathrm{V}$ as the Lagrangian mean velocity for EMM. The Stokes mean drift velocity is defined by [12],

$$
\langle\mathbf{U}\rangle^{S} \equiv\left\langle\zeta \cdot \nabla \mathbf{U}^{\prime}\right\rangle^{E} .
$$

Hence, equation (12.5) implies

$$
\langle\mathbf{U}\rangle^{S}=-\langle\boldsymbol{\zeta} \cdot \nabla \boldsymbol{\zeta} \cdot \nabla\rangle^{E} \overline{\mathbf{U}}=-\tilde{\Delta}^{E} \overline{\mathbf{U}}+o\left(|\boldsymbol{\zeta}|^{2}\right),
$$

where

$$
\tilde{\Delta}^{E} \equiv\left(\partial_{k}\left\langle\zeta^{k} \zeta^{l}\right\rangle^{E} \partial_{l}\right)=\left.\tilde{\Delta}_{D}^{E}\right|_{D=1},
$$

and we again argue that $\boldsymbol{\nabla} \cdot \boldsymbol{\zeta}=o\left(|\boldsymbol{\zeta}|^{2}\right)$. Thus, we find that $\mathbf{V}$ satisfies, to order $o\left(|\boldsymbol{\zeta}|^{2}\right)$,

$$
\mathbf{V} \equiv \overline{\mathbf{U}}-\tilde{\Delta}^{E} \overline{\mathbf{U}}=\overline{\mathbf{U}}+\langle\mathbf{U}\rangle^{S}=\langle\mathbf{U}\rangle^{L}
$$

Therefore, to this order, $\mathrm{V}$ is the Lagrangian mean velocity for the EMM theory. Thus, the duality between the Lagrangian mean velocity and the Eulerian mean velocity is reciprocated. In Eulerian mean theories the dual momentum is the Lagrangian mean velocity, and vice versa.

Contrasting the Euler-Poincaré equation (12.14) with the CL equation. The EMM motion equation (12.14) may be rewritten equivalently as

$$
\left(\frac{\partial}{\partial t}+\mathbf{V} \cdot \boldsymbol{\nabla}\right) \mathbf{V}+\underbrace{(\mathbf{V}-\overline{\mathbf{U}}) \times \operatorname{curl} \mathbf{V}}+\nabla P-\frac{1}{2}\left\langle\zeta^{k} \zeta^{l}\right\rangle^{E} \boldsymbol{\nabla}\left(\overline{\mathbf{U}}_{, k} \cdot \overline{\mathbf{U}}_{, l}\right)=0 .
$$

\section{Stokes vortex force}

Thus, the Stokes mean drift velocity $\mathbf{V}-\overline{\mathbf{U}}=\tilde{\Delta}^{E} \overline{\mathbf{U}}$ contributes an additional vortex force in this motion equation for the Lagrangian mean fluid velocity $\mathbf{V}$. This equation is similar in form to the CL equation (4.26). However, the meaning of the velocities are reversed in the two cases: the CL equation is for the Eulerian mean velocity $\overline{\mathbf{U}}$; and the EMM equation is for the Lagrangian mean velocity $\mathbf{V}$. The Stokes mean drift velocity is also prescribed for the CL equation, rather than being determined dynamically. 


\subsection{Kelvin circulation theorem for the Eulerian mean model}

Being Euler-Poincaré, the Eulerian mean model (EMM) equation (12.14) has a corresponding Kelvin-Noether circulation theorem. Namely, this equation implies, cf. equation (6.32),

$$
\frac{d}{d t} \oint_{\gamma(\overline{\mathbf{U}})} \mathbf{V} \cdot d \mathbf{x}=-\frac{1}{2} \iint_{S(\overline{\mathbf{U}})} \nabla\left(\overline{\mathbf{U}}_{, k} \cdot \overline{\mathbf{U}}_{, l}\right) \times \nabla\left\langle\zeta^{k} \zeta^{l}\right\rangle^{E} \cdot d \mathbf{S}
$$

for any closed curve $\gamma(\overline{\mathbf{U}})$ that moves with the Eulerian mean fluid velocity $\overline{\mathbf{U}}$ and surface $S(\overline{\mathbf{U}})$ with boundary $\gamma(\overline{\mathbf{U}})$. Thus, in this Kelvin-Noether circulation theorem the presence of the Eulerian mean fluctuation covariance $\left\langle\zeta^{k} \zeta^{l}\right\rangle^{E}$ creates circulation of the Lagrangian mean velocity $\mathbf{V}=\overline{\mathbf{U}}-\tilde{\Delta}^{E} \overline{\mathbf{U}}$.

\subsection{Vortex stretching equation for the Eulerian mean model}

In three dimensions, the EMM equation (12.14) may be expressed in its equivalent "curl" form, as

$$
\frac{\partial}{\partial t} \mathbf{V}-\overline{\mathbf{U}} \times(\boldsymbol{\nabla} \times \mathbf{V})+\boldsymbol{\nabla}\left(P_{t o t}^{E}+\overline{\mathbf{U}} \cdot \mathbf{V}\right)=-\frac{1}{2}\left(\overline{\mathbf{U}}_{, k} \cdot \overline{\mathbf{U}}_{, l}\right) \boldsymbol{\nabla}\left\langle\zeta^{k} \zeta^{l}\right\rangle^{E}, \quad \boldsymbol{\nabla} \cdot \overline{\mathbf{U}}=0 .
$$

The curl of this equation in turn yields an equation of transport and creation for the Lagrangian mean vorticity, $\mathbf{Q} \equiv \operatorname{curl} \mathbf{V}$,

$$
\frac{\partial \mathbf{Q}}{\partial t}+\overline{\mathbf{U}} \cdot \nabla \mathbf{Q}=\mathbf{Q} \cdot \nabla \overline{\mathbf{U}}-\frac{1}{2} \boldsymbol{\nabla}\left(\overline{\mathbf{U}}_{, k} \cdot \overline{\mathbf{U}}_{, l}\right) \times \nabla\left\langle\zeta^{k} \zeta^{l}\right\rangle^{E}, \quad \text { where } \quad \mathbf{Q} \equiv \operatorname{curl} \mathbf{V},
$$

and we have used incompressibility of $\overline{\mathbf{U}}$. Thus, $\overline{\mathbf{U}}$ is the transport velocity for the generalized vorticity $\mathbf{Q}$ and the expected vortex stretching term $\mathbf{Q} \cdot \nabla \overline{\mathbf{U}}$ is accompanied by an additional vortex creation term. Of course, this additional term is also responsible for the creation of circulation of $\mathbf{V}$ in the Kelvin-Noether circulation theorem (12.22) and it vanishes when the Eulerian mean covariance is homogeneous in space, thereby recovering the corresponding result for the three dimensional $\mathrm{CH}$ equation [1], [2].

\subsection{Energetics of the Eulerian mean model}

Noether's theorem guarantees conservation of energy for the Euler-Poincaré equations (12.14), since the Eulerian mean Lagrangian $\langle L\rangle^{E}$ in equation (12.9) has no explicit dependence on time. This constant energy is given by

$$
E=\frac{1}{2} \int d^{3} x\left(|\overline{\mathbf{U}}|^{2}+\left\langle\zeta^{k} \zeta^{l}\right\rangle^{E} \overline{\mathbf{U}}_{, k} \cdot \overline{\mathbf{U}}_{, l}\right)=\frac{1}{2} \int d^{3} x \overline{\mathbf{U}} \cdot \mathbf{V} .
$$

Thus, the total kinetic energy is the integrated product of the Eulerian mean and Lagrangian mean velocities. In this kinetic energy, the Eulerian mean covariance 
of the fluctuations couples to the gradients of the Eulerian mean velocity. So there is a cost in kinetic energy for the system either to increase these gradients, or to increase the Eulerian mean covariance.

As one might expect from the analysis in Section 0, Legendre transforming the Lagrangian $\langle L\rangle^{E}$ in $(12.9)$ gives the following Hamiltonian (still expressed in terms of the velocity $\overline{\mathbf{U}}$, instead of the momentum density $\left.\mathbf{M}=\delta\langle L\rangle^{E} / \delta \overline{\mathbf{U}}=D \mathbf{V}\right)$,

$$
H^{E}=\int_{\mathcal{M}} d^{n} x\left[\frac{D}{2}\left(|\overline{\mathbf{U}}|^{2}+\left\langle\zeta^{k} \zeta^{l}\right\rangle^{E} \overline{\mathbf{U}}_{, k} \cdot \overline{\mathbf{U}}_{, l}\right)+P(D-1)\right] .
$$

Remark on the geodesic property of the EMM model. When evaluated on the constraint manifold $D=1$, the Lagrangian in (12.9) and the Hamiltonian in (12.26) for the Eulerian mean fluid motion equation (12.14) coincide in $n$ dimensions. This is expected for a stationary principle giving rise to geodesic motion. The interpretation of the EMM model as describing geodesic motion on the volume preserving diffeomorphism group with respect to the $H_{1}$ metric given by (12.25) is discussed in 23] for the $\mathrm{CH}$ case, in which the Eulerian mean covariance is isotropic and homogeneous. The corresponding discussion for the EMM equations is equivalent to that given in 23], since the metrics are equivalent, provided the initial conditions for $\left\langle\zeta^{k} \zeta^{l}\right\rangle^{E}$ are bounded away from zero.

\subsection{Momentum conservation - stress tensor formulation}

Noether's theorem also guarantees conservation of momentum for the Euler-Poincaré equations (12.14), since the Eulerian mean Lagrangian $\langle L\rangle^{E}$ in equation (12.9) has no explicit spatial dependence. As before, the integrand $\mathcal{L}$ in this Lagrangian is a polynomial in the Lagrangian mean velocity $\overline{\mathbf{U}}$, its gradient $\overline{\mathbf{U}}_{, k}$, and the advected quantities $D$ and $\left\langle\zeta^{k} \zeta^{l}\right\rangle^{E}$. That is,

$$
\langle L\rangle^{E}=\int d^{3} x \mathcal{L}\left(\overline{\mathbf{U}}, \overline{\mathbf{U}}_{, k}, D,\left\langle\zeta^{k} \zeta^{l}\right\rangle^{E}\right),
$$

with $\mathcal{L}$ a polynomial function of its arguments. In this case, we may express the Eulerian mean Euler-Poincaré equations (12.14) in the momentum conservation form,

$$
\frac{\partial M_{i}}{\partial t}=-\frac{\partial}{\partial x^{j}} \mathcal{T}_{i}^{j}
$$

with momentum density components $M_{i}, i=1,2,3$ defined by

$$
M_{i} \equiv \frac{\delta\langle L\rangle^{E}}{\delta \bar{U}^{i}}=\frac{\partial \mathcal{L}}{\partial \bar{U}^{i}}-\frac{\partial}{\partial x^{k}}\left(\frac{\partial \mathcal{L}}{\partial \bar{U}_{, k}^{i}}\right),
$$

and stress tensor $\mathcal{T}_{i}^{j}$ given by, cf. equation (6.14),

$$
\mathcal{T}_{i}^{j}=M_{i} \bar{U}^{j}-\frac{\partial \mathcal{L}}{\partial \bar{U}_{, j}^{k}} \bar{U}_{, i}^{k}+\delta_{i}^{j}\left(\mathcal{L}-D \frac{\partial \mathcal{L}}{\partial D}\right)
$$


Equation (12.28) then implies conservation of the total momentum, $\int \mathbf{M} d^{3} x$, provided the normal component of the stress tensor $\mathcal{T}_{i}^{j}$ vanishes on the boundary.

In our particular case, expression (12.30) for the stress tensor $\mathcal{T}_{i}^{j}$ becomes

$$
\mathcal{T}_{i}^{j}=M_{i} \bar{U}^{j}-D \overline{\mathbf{U}}_{, k} \cdot \overline{\mathbf{U}}_{, i}\left\langle\zeta^{k} \zeta^{j}\right\rangle^{E}+P \delta_{i}^{j}, \quad \text { where } \quad M_{i}=D\left(\bar{U}_{i}-\tilde{\Delta}_{D}^{E} \bar{U}_{i}\right) .
$$

Consequently, the equivalent Euler-Poincaré motion equation (12.14), or (12.23) is also expressible as

$$
\frac{\partial V_{i}}{\partial t}=-\frac{\partial}{\partial x^{j}}\left(V_{i} \bar{U}^{j}+P \delta_{i}^{j}-\overline{\mathbf{U}}_{, k} \cdot \overline{\mathbf{U}}_{, i}\left\langle\zeta^{k} \zeta^{j}\right\rangle^{E}\right), \quad \text { with }\left.\quad V_{i} \equiv M_{i}\right|_{D=1} .
$$

The boundary conditions are given in equation (12.16).

\subsection{A second moment turbulence closure model for EMM}

When dissipation and forcing are added to the EMM motion equation (12.14) by using the phenomenological viscosity $\nu \tilde{\Delta}^{E} \mathbf{V}$ and forcing $\mathbf{F}$, one finds a second moment Eulerian mean turbulence model given by

$$
\begin{aligned}
\left(\frac{\partial}{\partial t}+\overline{\mathbf{U}} \cdot \boldsymbol{\nabla}\right) \mathbf{V}+V_{j} \boldsymbol{\nabla} \bar{U}^{j} & +\nabla P_{t o t}^{E}+\frac{1}{2}\left(\overline{\mathbf{U}}_{, k} \cdot \overline{\mathbf{U}}_{, l}\right) \boldsymbol{\nabla}\left\langle\zeta^{k} \zeta^{l}\right\rangle^{E} \\
& =\nu \tilde{\Delta}^{E} \mathbf{V}+\mathbf{F}, \quad \text { where } \boldsymbol{\nabla} \cdot \overline{\mathbf{U}}=0,
\end{aligned}
$$

with viscous boundary conditions $\mathbf{V}=0, \overline{\mathbf{U}}=0$ at a fixed boundary. Note that the Eulerian mean fluctuation covariance $\left\langle\zeta^{k} \zeta^{j}\right\rangle^{E}$ appears in the dissipation operator $\tilde{\Delta}^{E}$. In the absence of the forcing $\mathbf{F}$ satisfying appropriate regularity conditions, this viscous EMM turbulence model dissipates the energy $E$ in equation (12.25) according to

$$
\frac{d E}{d t}=-\nu \int d^{3} x\left[\operatorname{tr}\left(\nabla \overline{\mathbf{U}}^{T} \cdot\langle\boldsymbol{\zeta} \boldsymbol{\zeta}\rangle^{E} \cdot \nabla \overline{\mathbf{U}}\right)+\tilde{\Delta}^{E} \overline{\mathbf{U}} \cdot \tilde{\Delta}^{E} \overline{\mathbf{U}}\right] .
$$

This negative definite energy dissipation law justifies adding viscosity with $\tilde{\Delta}^{E}$, instead of using the ordinary Laplacian operator.

\section{Geophysical applications of the Eulerian mean model}

\subsection{Eulerian mean Euler-Boussinesq equations}

Introducting rotation and stratification alters the averaged approximate Lagrangian $\langle L\rangle^{E}$ in equation (12.9) to the following expression,

$$
\begin{aligned}
\langle L\rangle_{E B}^{E}=\int d^{3} x & \left\{\frac{D}{2}\left[|\overline{\mathbf{U}}|^{2}+\left\langle\zeta^{k} \zeta^{l}\right\rangle^{E}\left(\overline{\mathbf{U}}_{, k} \cdot \overline{\mathbf{U}}_{, l}\right)\right]\right. \\
+ & D \mathbf{R}(\mathbf{x}) \cdot \overline{\mathbf{U}}-g b D z+P[1-D]\} .
\end{aligned}
$$


To account for buoyancy $b$ in the Euler-Poincaré equation (12.12), the right hand side should have the additional summand, $D^{-1} b_{, i} \delta\langle L\rangle_{E B}^{E} / \delta b$. Then, the Euler-Poincaré equation resulting from $\langle L\rangle_{E B}^{E}$ in (13.1) is

$$
\begin{aligned}
& \frac{\partial \mathbf{V}}{\partial t}+\overline{\mathbf{U}} \cdot \boldsymbol{\nabla} \mathbf{V}+V_{j} \boldsymbol{\nabla} \bar{U}^{j}-\overline{\mathbf{U}} \times \operatorname{curl} \mathbf{R}(\mathbf{x}) \\
&=-g b \hat{\mathbf{z}}-\boldsymbol{\nabla} P_{t o t}^{E}-\frac{1}{2}\left(\overline{\mathbf{U}}_{, k} \cdot \overline{\mathbf{U}}_{, l}\right) \boldsymbol{\nabla}\left\langle\zeta^{k} \zeta^{l}\right\rangle^{E}, \\
& \text { where } \quad \mathbf{V} \equiv\left(1-\tilde{\Delta}^{E}\right) \overline{\mathbf{U}}, \quad \text { with } \quad \tilde{\Delta}^{E} \equiv \boldsymbol{\nabla} \cdot\langle\boldsymbol{\zeta} \boldsymbol{\zeta}\rangle^{E} \cdot \boldsymbol{\nabla},
\end{aligned}
$$

and the Eulerian mean flow is incompressible, so $\boldsymbol{\nabla} \cdot \overline{\mathbf{U}}=0$. The auxiliary equations that complete this set are

$$
\left(\frac{\partial}{\partial t}+\overline{\mathbf{U}} \cdot \nabla\right) b=0, \quad\left(\frac{\partial}{\partial t}+\overline{\mathbf{U}} \cdot \nabla\right)\langle\zeta \zeta\rangle^{E}=0, \quad \frac{\partial D}{\partial t}+\nabla \cdot(D \mathbf{U})=0,
$$

These Eulerian mean Euler-Boussinesq (EMEB) equations describe the Eulerian mean effects of fluctuations on the ideal motion of a stratified incompressible fluid in a rotating reference frame. The motion equation for this system may be rewritten in curl form, as

$\frac{\partial}{\partial t} \mathbf{V}-\overline{\mathbf{U}} \times \operatorname{curl}(\mathbf{V}+\mathbf{R})+\nabla\left(P_{t o t}^{E}+\overline{\mathbf{U}} \cdot \mathbf{V}\right)+g b \boldsymbol{\nabla} z+\frac{1}{2}\left(\overline{\mathbf{U}}_{, k} \cdot \overline{\mathbf{U}}_{, l}\right) \boldsymbol{\nabla}\left\langle\zeta^{k} \zeta^{l}\right\rangle^{E}=0$.

The Kelvin-Noether circulation theorem for the EMEB model is given by,

$\frac{d}{d t} \oint_{\gamma(\overline{\mathbf{U}})}(\mathbf{V}+\mathbf{R}) \cdot d \mathbf{x}=-\iint_{S(\overline{\mathbf{U}})}\left[\frac{1}{2} \boldsymbol{\nabla}\left(\overline{\mathbf{U}}_{, k} \cdot \overline{\mathbf{U}}_{, l}\right) \times \nabla\left\langle\zeta^{k} \zeta^{l}\right\rangle^{E}+g \boldsymbol{\nabla} b \times \boldsymbol{\nabla} z\right] \cdot d \mathbf{S}$.

Here, the closed curve $\gamma(\overline{\mathbf{U}})$ moves with the Eulerian mean fluid velocity $\overline{\mathbf{U}}$ and is the boundary of the surface $S(\overline{\mathbf{U}})$. There are two vorticity creation terms in the Kelvin-Noether circulation theorem (13.6). Thus, spatial variation in the Eulerian mean covariance and nonvertical buoyancy gradients can both create circulation of the sum $\mathbf{V}+\mathbf{R}$ around closed fluid loops moving with velocity $\overline{\mathbf{U}}$. Naturally, the same vorticity creation terms appear in the dynamics of the Lagrangian mean vorticity $\mathbf{Q}=\operatorname{curl} \mathbf{V}$, which may be obtained by taking the curl of equation (13.5).

The conserved energy for the EMEB system (13.2) - (13.3) is given by a formula similar to equation (10.7) for the LMEB model, which is

$$
E_{E M E B}=\int d^{3} x\left[\frac{1}{2}\left(|\overline{\mathbf{U}}|^{2}+\operatorname{tr}\left(\nabla \overline{\mathbf{U}}^{\mathrm{T}} \cdot\langle\zeta \zeta\rangle^{E} \cdot \nabla \overline{\mathbf{U}}\right)\right)+g b z\right] .
$$

Adding dissipation semiempirically as before yields equations (1.18) - (1.19) in the Introduction. 


\subsection{D Eulerian mean rotating shallow water (EMRSW)}

We apply the standard shallow water approximations to the averaged approximated EMEB Lagrangian $\langle L\rangle^{E}$ in equation (13.1) to find a new Lagrangian $\langle L\rangle_{R S W}^{E}$ for the dynamics of Eulerian mean rotating shallow water (EMRSW),

$$
\begin{aligned}
\langle L\rangle_{R S W}^{E}=\int d x d y\{ & \frac{D}{2}\left[|\overline{\mathbf{U}}|^{2}+\left\langle\zeta^{k} \zeta^{l}\right\rangle^{E}\left(\overline{\mathbf{U}}_{, k} \cdot \overline{\mathbf{U}}_{, l}\right)\right] \\
& \left.+D \mathbf{R}(\mathbf{x}) \cdot \overline{\mathbf{U}}-\frac{1}{2} g D^{2}+g D B(x, y)\right\} .
\end{aligned}
$$

Here $D=h+B(x, y)$ is the total depth of the water, which satisfies the continuity equation,

$$
\frac{\partial D}{\partial t}+\nabla \cdot(D \overline{\mathbf{U}})=0
$$

and the Eulerian mean covariance $\langle\xi \xi\rangle^{E}$ satisfies the dynamics inherited from the Taylor-like hypothesis (12.4),

$$
\left(\frac{\partial}{\partial t}+\overline{\mathbf{U}} \cdot \nabla\right)\langle\zeta \zeta\rangle^{E}=0 .
$$

The Euler-Poincaré motion equation (12.12) for EMRSW dynamics generated by the Lagrangian $\langle L\rangle_{R S W}^{E}$ is expressed as

$$
\begin{aligned}
\left(\frac{\partial}{\partial t}+\overline{\mathbf{U}} \cdot \boldsymbol{\nabla}\right) \mathbf{V} & -\overline{\mathbf{U}} \times \operatorname{curl} \mathbf{R}(\mathbf{x})+g \boldsymbol{\nabla}(D-B(x, y)) \\
& =-V_{j} \boldsymbol{\nabla} \bar{U}^{j}-\frac{1}{2}\left(\overline{\mathbf{U}}_{, k} \cdot \overline{\mathbf{U}}_{, l}\right) \boldsymbol{\nabla}\left\langle\zeta^{k} \zeta^{l}\right\rangle^{E},
\end{aligned}
$$

where the Eulerian mean circulation velocity $\mathbf{V}=\left(1-\tilde{\Delta}_{D}^{E}\right) \overline{\mathbf{U}}$ is defined as in equation (12.15), but now in $2 \mathrm{D}$. The left hand side of this equation is the same as the standard motion equation for rotating ideal shallow water dynamics, modulo the substitution $\overline{\mathbf{U}} \rightarrow \mathbf{V}$ and the additional dynamics for the covariance $\langle\zeta \zeta\rangle^{E}$. The right hand side has a "line element stretching term" and a "covariance gradient term."

This motion equation for EMRSW implies the Kelvin-Noether circulation theorem, cf. equation (12.22),

$$
\frac{d}{d t} \oint_{\gamma(\overline{\mathbf{U}})}(\mathbf{V}+\mathbf{R}) \cdot d \mathbf{x}=-\frac{1}{2} \iint_{S(\overline{\mathbf{U}})}\left[\nabla\left(\overline{\mathbf{U}}_{, k} \cdot \overline{\mathbf{U}}_{, l}\right) \times \nabla\left\langle\zeta^{k} \zeta^{l}\right\rangle^{E}\right] \cdot d \mathbf{S},
$$

with fluid loop $\gamma(\overline{\mathbf{U}})$ and surface $S(\overline{\mathbf{U}})$, as before. Thus, as for the EMM model, the covariance gradient term has the effect of generating (total) circulation. The curl of the EMRSW motion equation yields, with $Q=\hat{z} \cdot \operatorname{curl}(\mathbf{V}+\mathbf{R})$,

$$
\left(\frac{\partial}{\partial t}+\overline{\mathbf{U}} \cdot \nabla\right)\left(\frac{Q}{D}\right)=-\frac{1}{2 D} \hat{\mathbf{z}} \cdot \nabla\left(\overline{\mathbf{U}}_{, k} \cdot \overline{\mathbf{U}}_{, l}\right) \times \nabla\left\langle\zeta^{k} \zeta^{l}\right\rangle^{E} .
$$


Thus, the potential vorticity $Q / D$ is not conserved on fluid parcels by the EMRSW model, but instead has a local creation term proportional to $\nabla\left\langle\zeta^{k} \zeta^{l}\right\rangle^{E}$. However, conservation of circulation on fluid loops and conservation of potential vorticity $Q / D$ on fluid parcels is recovered for the invariant subsystem $\langle\zeta \zeta\rangle^{E}=\alpha^{2} \delta^{k l}$ with constant $\alpha^{2}$. For this invariant subsystem, we have $\mathbf{V}=\left(1-\alpha^{2} \Delta\right) \overline{\mathbf{U}}$ (with the ordinary Laplacian operator) and the covariance gradient $\nabla\left\langle\zeta^{k} \zeta^{l}\right\rangle^{E}$ vanishes. Hence, the right hand sides of equations (13.12) and (13.13) also vanish in this case; so that the Kelvin circulation integrals are constant and the potential vorticity is conserved on fluid parcels in this case.

\subsection{D Eulerian mean shallow water model}

Restricting the Lagrangian $\langle L\rangle_{R S W}^{E}$ in equation (13.8) to one dimensional motion (without rotation) results in

$$
\langle L\rangle_{S W}^{E}=\int d x\left[\frac{D}{2}\left(\bar{U}^{2}+W \bar{U}_{x}^{2}\right)-\frac{1}{2} g D^{2}+g D B(x)\right] .
$$

Here $W$ denotes $\langle\zeta \zeta\rangle^{E}$ in 1D, subscripts denote partial derivatives and $D=h+B(x)$ is the depth of the water. The corresponding Euler-Poincaré equation is, from equation (12.12),

$$
V_{t}+\bar{U} V_{x}+V \bar{U}_{x}+\frac{1}{2} W_{x} \bar{U}_{x}^{2}+\left[g(D-B(x))-\frac{1}{2}\left(\bar{U}^{2}+W \bar{U}_{x}^{2}\right)\right]_{x}=0 .
$$

This may also be written in momentum conservation form for EMSW in 1D as, cf. equations (12.28) - (12.30),

$$
\begin{aligned}
M_{t}=-\left(M \bar{U}-D W \bar{U}_{x}^{2}+\right. & \left.\frac{1}{2} g D^{2}\right)_{x}+g D B_{x} \\
\text { with } M \text { defined by } M & \equiv \frac{\delta\langle L\rangle_{S W}^{E}}{\delta \bar{U}}=D \bar{U}-\left(D W \bar{U}_{x}\right)_{x}, \\
\text { where } D \text { and } W \text { satisfy } D_{t} & =-(D \bar{U})_{x}, \\
\text { and } W_{t} & =-\bar{U} W_{x} .
\end{aligned}
$$

The equivalent equations (13.15) and (13.16) simplify into the following motion equation for the Lagrangian mean velocity $V=M / D$,

$$
\begin{aligned}
V_{t}+\bar{U} V_{x}-D^{-1}\left(D W \bar{U}_{x}^{2}\right)_{x} & =-g(D-B(x))_{x} \\
\text { where } V=\bar{U} & -D^{-1}\left(D W \bar{U}_{x}\right)_{x} .
\end{aligned}
$$

Thus, the Eulerian mean equation for one dimensional shallow water makes the change $V \rightarrow \bar{U}$ in the advection term, and introduces another term on the left hand side. Amusingly, this other term is half the commutator of the advective derivative $d / d t$ and the Helmholtz operator $1-\tilde{\Delta}_{D}^{E}$ acting on $\bar{U}$. That is,

$$
2 D^{-1}\left(D W \bar{U}_{x}^{2}\right)_{x}=\left[\left(\frac{\partial}{\partial t}+\bar{U} \frac{\partial}{\partial x}\right),\left(1-D^{-1} \frac{\partial}{\partial x} D W \frac{\partial}{\partial x}\right)\right] \bar{U} .
$$


Therefore, we have two equivalent forms of the 1D EMSW equations

$$
\begin{aligned}
V_{t}+\bar{U} V_{x}-D^{-1}\left(D W \bar{U}_{x}^{2}\right)_{x} & =-g(D-B(x))_{x}, \\
\left(1-\tilde{\Delta}_{D}^{E}\right)\left(\bar{U}_{t}+\bar{U} \bar{U}_{x}\right)+D^{-1}\left(D W \bar{U}_{x}^{2}\right)_{x} & =-g(D-B(x))_{x}, \\
\text { where } V=\left(1-\tilde{\Delta}_{D}^{E}\right) \bar{U}=\bar{U} & -D^{-1}\left(D W \bar{U}_{x}\right)_{x} .
\end{aligned}
$$

Hence, the one dimensional Eulerian mean shallow water system may be rewritten as, cf. equation (11.16),

$$
\begin{aligned}
& \bar{U}_{t}+\bar{U} \bar{U}_{x}+\left(1-\tilde{\Delta}_{D}^{E}\right)^{-1}\left[g(D-B)_{x}+D^{-1}\left(D W \bar{U}_{x}^{2}\right)_{x}\right]=0 \\
& D_{t}+\bar{U} D_{x}+D \bar{U}_{x}=0, \quad W_{t}+\bar{U} W_{x}=0 \\
& \left(1-\tilde{\Delta}_{D}^{E}\right) \equiv\left(1-D^{-1} \partial_{x} D W \partial_{x}\right)
\end{aligned}
$$

This system for $W \neq 0$ is no longer hyperbolic; rather, it is conservative and dispersive. When $W$ is constant, we obtain the shallow water alpha-model as an invariant subsystem.

The equations for Eulerian mean polytropic gas dynamics with pressuredensity relation $p=p_{0}\left(D / D_{0}\right)^{\gamma}$, are obtained by replacing the motion equation in the Eulerian mean shallow water system $(13.25)-(13.27)$ with

$$
\bar{U}_{t}+\bar{U} \bar{U}_{x}+\left(1-\tilde{\Delta}_{D}^{E}\right)^{-1}\left[\frac{p_{0} \gamma}{D_{0}^{\gamma}} D^{\gamma-2} D_{x}+D^{-1}\left(D W \bar{U}_{x}^{2}\right)_{x}\right]=0 .
$$

Thus, equations (13.26) - (13.28) provide a system of Eulerian mean polytropic gas equations, which is no longer hyperbolic for $W \neq 0$, and which recovers the polytropic gas alpha model as an invariant subsystem when $W$ is constant. This system conserves the energy,

$$
E=\int d x\left[\frac{D}{2}\left(\bar{U}^{2}+W \bar{U}_{x}^{2}\right)+\frac{p_{o}}{\gamma-1} \frac{D^{\gamma}}{D_{0}^{\gamma}}\right] .
$$

\subsection{The Eulerian mean Riemann (EMR) equation}

For another illustration of the Eulerian mean methodology in one dimension, we return to the Riemann equation,

$$
\bar{U}_{t}+3 \bar{U} \bar{U}_{x}=0
$$

This is the Euler-Poincaré equation for the Lagrangian,

$$
L_{R}=\int d x \frac{1}{2} \bar{U}^{2}
$$

The corresponding Eulerian mean approximate Lagrangian for this problem is (again writing $\langle\zeta \zeta\rangle^{E}=W$ for the Eulerian mean covariance in one dimension)

$$
\langle L\rangle_{R}^{E}=\int d x \frac{1}{2}\left(\bar{U}^{2}+W \bar{U}_{x}^{2}\right),
$$


whose Euler-Poincaré equation is

$$
V_{t}+\bar{U} V_{x}+2 V \bar{U}_{x}+\frac{1}{2} W_{x} \bar{U}_{x}^{2}=0
$$

with

$$
V=\frac{\delta\langle L\rangle_{R}^{E}}{\delta \bar{U}}=\bar{U}-\left(W \bar{U}_{x}\right)_{x}
$$

where the one dimensional Eulerian mean covariance $W$ satisfies the scalar advection law

$$
W_{t}=-\bar{U} W_{x}
$$

The Eulerian mean Riemann system (13.33) - (13.35) can be rewritten in momentum conservation form as

$$
V_{t}+\left(\frac{1}{2} \bar{U}^{2}-\frac{W}{2} \bar{U}_{x}^{2}+\bar{U} V\right)_{x}=0,
$$

and in nonlocal characteristic form as

$$
\begin{aligned}
\bar{U}_{t}+\bar{U} \bar{U}_{x} & =-\left(1-\partial_{x} W \partial_{x}\right)^{-1} \partial_{x}\left(\bar{U}^{2}+\frac{W}{2} \bar{U}_{x}^{2}\right), \\
\text { and } W_{t} & =-\bar{U} W_{x} .
\end{aligned}
$$

We compare the Eulerian mean Riemann system (13.33) - (13.35) with the completely integrable model of Camassa-Holm [21], 222] for nonlinearly dispersive shallow water waves. The equation of motion for the $\mathrm{CH}$ model can be written in momentum conservation form as, cf. equation (13.36),

$$
\left(\bar{U}-\bar{U}_{x x}\right)_{t}+\left(\frac{1}{2} \bar{U}^{2}-\frac{1}{2} \bar{U}_{x}^{2}+\bar{U}\left(\bar{U}-\bar{U}_{x x}\right)\right)_{x}=0 .
$$

Moreover, the $\mathrm{CH}$ equation may also be written in nearly the same (nonlocal) characteristic form as equation (13.37) for the EMR problem,

$$
\bar{U}_{t}+\bar{U} \bar{U}_{x}=-\left(1-\partial_{x}^{2}\right)^{-1} \partial_{x}\left(\bar{U}^{2}+\frac{1}{2} \bar{U}_{x}^{2}\right) .
$$

The $\mathrm{CH}$ equation (13.39) is the Euler-Poincaré equation for the Lagrangian

$$
L_{C H}=\int d x \frac{1}{2}\left(\bar{U}^{2}+\bar{U}_{x}^{2}\right) .
$$

Thus, the Eulerian mean Riemann system 113.33 - 13.35 reduces to the CH equation (13.39) for $W=1$ (or for any other nonzero constant, which can be absorbed into the spatial length scale). Hence, the Eulerian mean Riemann system is the natural extension of the $\mathrm{CH}$ model to allow for time dependence of the length scale associated with $W$, the Eulerian mean covariance of the rapid fluctuations. More analysis of this equation is given in [42]. 


\section{Conclusions}

We have considered two classes of models that describe the mean motion of a fluid in the presence of rapid or random fluctuations. These are:

- The Lagrangian mean fluid models, in which the fluctuation is modeled as a displacement of the Lagrangian fluid parcel trajectory and averages are taken holding its Lagrangian label fixed; and

- The Eulerian mean fluid models, in which the fluctuation is modeled as occurring at a fixed position, based on the traditional Reynolds decomposition of the fluid velocity, and averages are taken at fixed spatial position.

At linear order in a Taylor expansion in the magnitude of the fluctuations we obtain the relation (4.6) that allows us to treat the two classes of models on the same mathematical footing. We apply asymptotics and averaging methods to Hamilton's principle for an ideal fluid and use the Euler-Poincaré theory of Holm, Marsden and Ratiu [1], [2] to determine the equations of motion that result in each class of model.

From the Euler-Poincaré viewpoint, the two classes of models differ from each other primarily in the way they treat Taylor's hypothesis, that the fluctuations should be "frozen" into the mean flow. We take Taylor's hypothesis to mean that the fluctuations should "transform" in a certain way under the action of the mean flow. In the Eulerian mean models, the displacement fluctuation transforms under the action of the Eulerian mean flow as a collection of scalars, by parallel transport. (See [23] for a mathematical description of this action using parallel transport in a composition of diffeomorphisms in a manifold setting.) In the Lagrangian mean models, the displacement fluctuation transforms under the action of the Lagrangian mean flow as a vector field, by Lie transport as in equation (3.17).

The two different models yield different equations via the Euler-Poincaré theory. However, these equations are "dual" to each other in the physical interpretations of their solutions. Namely, the momentum evolving in the Lagrangian mean models is interpreted in Section 4 as the Eulerian mean velocity. And, vice versa, the momentum evolving in the Eulerian mean models is interpreted in Section 12 as the Lagrangian mean velocity. The energy in both models is the total mean kinetic energy. The formal source of this duality between the two classes of models turns out to be their shared form of conserved total mean kinetic energy. This kinetic energy in both cases may be interpreted as the domain-integrated product of the Eulerian mean velocity times the Lagrangian mean velocity. These two velocities are related in both classes of models by a dynamical Helmholtz operator whose metric is the covariance of the fluctuations. Evenness of this Helmholtz operator allows the kinetic energy to be written as the $H^{1}$ norm of whichever velocity is being studied. Then, the variational derivative of the kinetic energy with respect to one of these velocities summons the other one and thereby produces the duality, in their Euler-Poincaré equations.

The effect of the averaging in either case is to make the solution velocity smoother than the momentum, or circulation velocity that it transports, via the inversion of 
the Helmholtz operator that relates the two velocities. The covariance that provides the metric appearing in this Helmholtz operator introduces a length scale which evolves with the mean flow according to the appropriate Taylor hypothesis for the fluctuations. The magnitude of this length scale determines the smoothness of the solution velocity for both classes of models. The mechanism for the smoothing is nonlinear dispersion in these ideal fluid theories (before viscosity is added). The nonlinear dispersion contains the length scale associated with the covariance and acts to suppress the magnitude of the fluidic triad interaction at smaller length scales. Normally, the triad interaction in fluid dynamics drives the cascade of energy forward to smaller length scales. However, in both of the classes of mean fluid models we study here this process is suppressed by nonlinear dispersion. In summary,

The nonlinear dispersion in these mean fluid theories acts to make the transport velocity smoother than the circulation or momentum velocity, by the inversion of a Helmholtz operator whose length scale corresponds to the fluctuation covariance.

These mean theories each provide either an extension, or a development of the viscous Camassa-Holm equation (VCHE, or NS- $\alpha$ ) that has recently been introduced as a one-point turbulence closure model, [3]- [5]. The Eulerian mean models are indeed natural extensions of the VCHE, or NS- $\alpha$ models to second order closures for turbulence. The Eulerian mean models reduce to the VCHE, or NS- $\alpha$ model when the Eulerian mean fluctuation covariance is spatially homogeneous. The Lagrangian mean models are another departure which also provides a second moment closure for turbulence that is related to the VCHE, or NS- $\alpha$ model, but does not contain it as an invariant subsystem unless the covariance vanishes entirely. Which approach will eventually lead to an appropriate model for climate and other long time geophysical applications remains to be seen.

We also formulated several examples of these Eulerian mean and Lagrangian mean fluid models in fewer dimensions, in the hopes that these simpler examples will provide additional physical insight into the dynamical behavior of these two classes of models.

\section{Acknowledgements}

We are grateful to S.Y. Chen, J.K. Dukowicz, C. Foias, S. Ghosal, R. Jordan, R.H. Kraichnan, J.E. Marsden, J.R. Ristorcelli, S. Shkoller and E.S. Titi for constructive comments and enlightening discussions during the course of this work.

\section{References}

[1] D.D. Holm, J.E. Marsden, T.S. Ratiu, The Euler-Poincaré equations and semidirect products with applications to continuum theories, Adv. in Math. 137 (1998) 1. 
[2] D.D. Holm, J.E. Marsden, T.S. Ratiu, Euler-Poincare models of ideal fluids with nonlinear dispersion, Phys. Rev. Lett. 80 (1998) 4173-4177.

[3] S. Chen, C. Foias, D.D. Holm, E. Olson, E.S. Titi, S. Wynne, The CamassaHolm equations as a closure model for turbulent channel and pipe flow, Phys. Rev. Lett., 81 (1998) 5338-5341.

[4] S. Chen, C. Foias, D.D. Holm, E. Olson, E.S. Titi, S. Wynne, A connection between the Camassa-Holm equations and turbulent flows in channels and pipes, Phys. Fluids, to appear.

[5] S. Chen, C. Foias, D.D. Holm, E. Olson, E.S. Titi, S. Wynne, The CamassaHolm equations and turbulence, Physica D, to appear.

[6] D.D. Holm and S. Shkoller, Mean fluid motion on Riemannian manifolds, in preparation.

[7] D.D. Holm, Isopycnal hydrostatic mean fluid dynamics, in preparation.

[8] G.K. Batchelor, An Introduction to Fluid Dynamics (Cambridge University Press, 1970), p. 276.

[9] G.I. Taylor, The spectrum of turbulence, Proc. Roy. Soc. A 164 (1938) 476.

[10] J.O. Hinze, Turbulence, (Mc-Graw-Hill: New York, 2nd edition, 1975).

[11] W.J.A. Dahm and K.B. Southerland, Experimental assessment of Taylor's hypothesis and its applicability to dissipation estimates in turbulent flows, Phys. Fluids 9 (1997) 2101-2107.

[12] D.G. Andrews and M.E. McIntyre, An exact theory of nonlinear waves on a Lagrangian mean flow, J. Fluid Mech. 89 (1978) 609-646.

[13] D.G. Andrews and M.E. McIntyre, On wave action and its relatives, J. Fluid Mech. 89 (1978) 647-664. (Corrigendum 95 (1978) 796.)

[14] I. Gjaja and D.D. Holm, Self-consistent Hamiltonian dynamics of wave meanflow interaction for a rotating stratified incompressible fluid, Physica D 98 (1996) 343-378.

[15] D.D. Holm, The ideal Craik-Leibovich equations, Physica D, 98 (1996) 415-441.

[16] A.D.D. Craik, and S. Leibovich, A rational model for Langmuir circulations, $J$. Fluid Mech. 73 (1976) 401-426. 
[17] A.S. Monin and A.M. Yaglom, Statistical Fluid Dynamics, vols. 1,2, (MIT Press, 1971).

[18] G.I. Taylor, Diffusion by continuous movements, Proc. London Math. Soc. 20 (1921) 196-211.

[19] A.F. Bennett, Particle displacements in inhomogeneous turbulence, in Stochastic modelling in Physical Oceanography, R.J. Adler, P. Müller and B. Rozovskii, eds., Birkhaüser, (1996) 1-45.

[20] J.K. Dukowicz and D. D. Holm, in preparation.

[21] R. Camassa and D.D. Holm, An integrable shallow water equation with peaked solitons, Phys. Rev. Lett. 71 (1993) 1661-1664.

[22] R. Camassa, D.D. Holm and J.M. Hyman, A new integrable shallow water equation. Advances in Applied Mechanics, Academic Press: Boston, vol 31 (1994) pp 1-33.

[23] D.D. Holm, S. Kouranbaeva, J.E. Marsden, T. Ratiu and S. Shkoller, A nonlinear analysis of the averaged Euler equations, Fields Inst. Comm., Arnold Vol. 2, Amer. Math. Soc., (Rhode Island) (1998) to appear.

[24] S. Shkoller, Geometry and curvature of diffeomorphism groups with $H^{1}$ metric and mean hydrodynamics. J. Func. Anal. 160 (1998) 337-365.

[25] J.E. Dunn and R.L. Fosdick, Thermodynamics, stability, and boundedness of fluids of complexity 2 and fluids of second grade, Arch. Rat. Mech. Anal. 56 (1974) 191-252.

[26] J.E. Dunn and K.R. Rajagopal, Fluids of differential type: Critical reviews and thermodynamic analysis, Int. J. Engng. Sci. 33 (1995) 689-729.

[27] R.S. Rivlin, The relation between the flow of non-Newtonian fluids and turbulent Newtonian fluids, Q. Appl. Math. 15 (1957) 212-215.

[28] A.J. Chorin, Spectrum, dimension, and polymer analogies in fluid turbulence, Phys. Rev. Lett. 60 (1988) 1947-1949.

[29] T.H. Shih, J. Zhu and J.L. Lumley, A new Reynolds stress algebraic equation model, Comput. Methods Appl. Mech. Engng. 125 (1995) 287-302.

[30] A. Yoshizawa, Statistical analysis of the derivation of the Reynolds stress from its eddy-viscosity representation, Phys. Fluids 27 (1984) 1377-1387. 
[31] R. Rubinstein and J.M. Barton, Nonlinear Reynolds stress models and the renormalization group, Phys. Fluids A 2 (1990) 1472-1476.

[32] A.A. Townsend, The Structure of Turbulent Flows, (Cambridge University Press, 1967).

[33] J.L. Lumley and H. Tennekes, A First Course in Turbulence, (MIT Press, 1972).

[34] W. C. Reynolds, Fundamentals of turbulence for turbulence modelling and simulation, in: Lecture Notes for Von Karman Institute, AGARD Lecture Note Series, (NATO, New York, 1987) pp.1-66.

[35] U. Piomelli, Applications of large eddy simulations in engineering: an overview, in: Large-eddy Simulation of Complex Engineering and Geophysical Flows, ed. B. Galperin and S. A. Orszag (Cambridge University Press, 1993).

[36] M. Lesieur and O. Métais, New trends in large-eddy simulations of turbulence, Annual Rev. Fluid Mech. 28 (1996) 45-82.

[37] S. Chen, D.D. Holm, L.G. Margolin and R. Zhang, 3D DNS of the CamassaHolm equation and its interpretation as an LES model, in preparation.

[38] J. Leray, Acta Math. 63 (1934) 193. Reviewed, e.g., in G. Gallavotti, Some rigorous results about 3D Navier-Stokes, in Les Houches 1992 NATO-ASI meeting on Turbulence in Extended Systems, eds. R. Benzi, C. Basdevant and S. Ciliberto (Nova Science, New York, 1993) pp. 45-81. See also, P. Constantin, C. Foias, B. Nicolaenko and R. Temam, Integral manifolds and inertial manifolds for dissipative partial differential equations. Applied Mathematical Sciences, 70, (Springer-Verlag, New York-Berlin, 1989).

[39] C. Foias, D.D. Holm and E.S. Titi, Regularity of the viscous Camassa-Holm equations, in preparation.

[40] R.D. Smith, The primitive equations in the stochastic theory of adiabatic stratified turbulence, LA-UR-97-4319. J. Phys. Oceanog., to appear.

[41] R.E. Davis, Lagrangian ocean studies, Ann. Rev. Fluid Mech. 23 (1991) 43-64.

[42] D.D. Holm, The Eulerian and Lagrangian means of the Riemann equation, in preparation. 